\title{
Prodromus of moth flies (Diptera: Psychodidae) from Slovakia
}

\author{
Jozef Oboňa \& Jan Ježek
}

\begin{abstract}
Prodromus of moth flies (Diptera: Psychodidae) from Slovakia. - Acta Mus. Siles. Sci. Natur., 63: 193-251, 2014.

Abstract: First records of fourteen species of moth flies are from Slovakia mentioned: Apsycha pusilla (Tonnoir, 1922), Clytocerus (Boreoclytocerus) splendidus Ježek \& Hájek, 2007, C. (B.) tetracorniculatus Wagner, 1977, Jungiella (Jungiella) hygrophila Ježek, 1987, J. (J.) valachica (Vaillant, 1963), J. (Psychocha) laminata (Szabó, 1960), Parajungiella prikryli Ježek, 1999, Pericoma (Pericoma) exquisita Eaton, 1893, P. (P.) ljubiniensis Krek, 1969, P. (P.) vestita Vaillant \& Withers, 1993, Psychoda alticola Vaillant, 1973, P. uniformata Haseman, 1907, Pneumia compta (Eaton, 1893) and P. kabelaki Omelková \& Ježek, 2012. Records on the basis of larvae of Berdeniella manicata (Tonnoir, 1920), Pericoma (Pericoma) pseudoexquisita Tonnoir, 1940 and Pneumia stammeri (Jung, 1956) in the past are first time confirmed as adults from Slovakia. Some detailed important morphological characters males of two selected species Pericoma (Pericoma) exquisita and $P$. (P.) pseudoexquisita are figured. All credible and available data of non-biting moth flies (Diptera: Psychodidae) from Slovakia were summarized (396 localities) and many new collecting sites (visited by different collectors from 1977 to 2013) added (188 localities). A suitable grid mapping for Central Europe was used. A preliminary conservation status of 51 rare species is discussed. The speciesrichness of the psychodid fauna in Slovakia is increased by this paper to 117 species.
\end{abstract}

Key words: Diptera, Psychodidae, new records, new localities, complete summarization of faunistics, grid mapping, threatened species, Slovakia

\section{Introduction}

The investigation of Psychodidae in Slovakia is still far from finished. Scattered older literary data of moth flies (e.g. Thalhammer 1918, Szabó 1960, 1965a,b) were firstly summarized by Rozkošný (1971). This work raised the number of Psychodidae known from Slovakia to 27 species. Subsequently Halgoš (1984) increased the number of Psychodidae to 34 species, next Ježek \& Halgoš (1987) to 41 species known previously from Slovakia. Ježek (1997, 2003 and 2006) gave next summarization with 65, 73 and 89 species.

The recent checklist of non-biting moth flies from Slovakia (Ježek 2009c) includes 97 species. Ježek et al. (2012) published four new records for Slovakia: Yomormia furva (Tonnoir, 1940), invasive Clogmia albipunctata (Williston, 1893), Peripsychoda fusca (Macquart, 1826) and Pneumia gracilis gracilis (Eaton, 1893). Together with dendrolimnetic moth flies: Lepiseodina tristis (Meigen, 1830) and Sciria advena (Eaton, 1893) (Oboňa \& Ježek 2012b), the Slovakian fauna now comprises 117 species with the present paper's new records: Apsycha pusilla (Tonnoir, 1922), Clytocerus (Boreoclytocerus) splendidus Ježek \& Hájek, 2007, C. (B.) tetracorniculatus Wagner, 1977, Jungiella (Jungiella) hygrophila Ježek, 1987, J. (J.) valachica (Vaillant, 1963), J. (Psychocha) laminata (Szabó, 1960), Parajungiella prikryli Ježek, 1999, Pericoma (Pericoma) exquisita Eaton, 1893, P. (P.) ljubiniensis Krek, 1969, P. (P.) vestita Vaillant \& Withers, 1993, Psychoda alticola Vaillant, 1973, $P$. uniformata Haseman, 1907, Pneumia compta (Eaton, 1893) and P. kabelaki Omelková \& Ježek, 2012. 


\section{Material and methods}

Most of the new material was collected by several authors and a variety of methods, mainly by sweep netting and rarely with hand collecting, Malaise traps, rearing or yellow pan traps. The captured moth flies were preserved in 75\% ethanol, and mounted on 737 slides (Canada balsam) in Prague. These are now deposited in the Department of Entomology of the National Museum in Prague, Czech Republic (NMPC). All known credible and available data (incl. historical records) from random and extensive sampling of moth flies (Diptera: Psychodidae) from Slovakia were summarized: many thousands of specimens of moth flies were collected altogether at 396 localities situated in Slovakia - map 1 (see List of collecting sites). Number of localities published only in the past - map 2: 220, new localities - map 3: 188 (this data are chronologically arranged). Identification and nomenclature were used according to Vaillant (1971-1983), Ibañez-Bernal (2008), Ježek (1994, 2009a) and Ježek \& Van Harten (2009). A preliminary assessment of the current conservation status of rare species of moth flies in this paper was confronted and compared with the red list in the Czech Republic (Ježek 2005).

The following abbreviations are used: CR - Critically Endangered, EN - Endangered, F - female, HC hand collecting, Imts. - inter mountains, M - male, MT - Malaise trap, Mts. - mountains, NMPC entomological collections of the National Museum in Prague, Czech republic, NP - National Park, NS Nationally Scarce, SW - sweep netting, veg. - vegetation, TE - Tulgren's equipment, VU - Vulnerable, YPT yellow pan traps.

\section{List of collecting sites}

Each entry in the list of collecting sites is arranged as follows: number of locality, name of the collecting site (name of the nearest village or town), geomorphological units and grid mapping codes of the Databank of the Slovak fauna (DFS): cited following Mazúr et al. (1986), Lučivjanská (1989) and Stloukal \& Grujbárová (2013).

1. Alabastrová jaskyňa cave, Belianske Tatry Mts. NP, 6787.

2. Antalova studnička well, Veporské vrchy Mts., 7284.

3. Babí potok brook env. Ždiar, Belianske Tatry Mts. NP, 6787.

4. Babia hora Mt. env. Bystré, Oravské Beskydy Mts., 6483.

5. Bábsky les forest nr. Vel'ký Báb, Nitrianska pahorkatina hills, 7673.

6. Baláže, brook, Nízke Tatry Mts. NP, 7181.

7. Bánovo, Veporské vrchy Mts., 7284.

8. Banské, stream, Slanské vrchy Mts., 7195.

9. Bansko brook nr. Liptovská Lúžna, Nízke Tatry Mts. NP, 7081.

10. Baranie, Čergov Mts., 6892.

11. Bardejov, Ondavská vrchovina hills, 6693.

12. Bardejovské Kúpele, brook, Ondavská vrchovina hills, 6693.

13. Batizovce, brook, Popradská kotlina basin, 6987.

14. Belá nad Cirochou, meander, Laborecká vrchovina hills, 7098.

15. Biela Orava river nr. Lokca, Podbeskydská vrchovina Mts., 6682.

16. Biela, Podtatranská brázda furrow, 6787.

17. Biely Váh river, Vysoké Tatry Mts. NP, 6886.

18. Bodrog river basin*.

19. Bratislava, Podunajská rovina flat, 7868.

20. Brehy, Vtáčnik Mts., 7577.

21. Bretka, Rimavská kotlina basin, 7588 .

22. Brezno, Horehronské podolie basin, 7183.

23. Brtkovica nr. Liptovský Mikuláš, Nízke Tatry Mts. NP, 6984.

24. Brusno env. Brezno, brook, Nízke Tatry Mts. NP, 7982.

25. Bukovce env. Stropkov, brook, Ondavská vrchovina hills, 6796.

26. Bukovec nr. Donovaly, Starohorské vrchy Mts., 7181.

27. Bukovecká dolina valley env. Ráztoka, Nízke Tatry Mts. NP, 7182.

28. Bukovinka well, Vel'ká Fatra Mts. NP, 6981

29. Bystrá, Banská Bystrica distr., Horehronské podolie basin, 7183.

30. Čaklov, stream, Východoslovenská pahorkatina hills, 7095.

31. Čermel’ nr. Košice, brook, swamps, Čierna hora Mt., 7293.

32. Červená Magura, Nízke Tatry Mts. NP, 7081.

33. Červenica, swamps, Slanské vrchy Mts., 7194.

34. Červený potok brook - Nová Lesná, Popradská kotlina basin, 6887. 
35. Čičarovce, Východoslovenská rovina flat, 7489.

36. Čierna dolina valley env. Tatranská Kotlina, Popradská kotlina basin, 6787.

37. Čierna Lehota, Revúcka vrchovina Mts., 7287.

38. Čierny Diel env. Ráztoka, spring area, Nízke Tatry Mts. NP, 7182.

39. Čierny Hron brook - Dobročský prales Wirgin Forest, Veporské vrchy Mts., 7384.

40. Čierny Hron, Veporské vrchy Mts., 7183.

41. Čírč, Spišsko-šarišské medzihorie Imts., 6791.

42. Črmné, Nízke Tatry Mts. NP, 7183.

43. Danielov dom house nr. Nová Polianka, Popradská kotlina basin, 6886.

44. Dedačov, brook, Laborecká vrchovina hills, 6997.

45. Demänovská dolina valley, Nízke Tatry Mts. NP, 6983.

46. Diviacka Nová Ves, Strážovské vrchy Mts., 7276.

47. Dlhý Grún̆ nr. Čierny Balog, Veporské vrchy Mts., 7284.

48. Dobšinská l'adová jaskyňa cave, Slovenský raj NP, 7187.

49. Dolina 7 prameňov valley nr. Tatranská Kotlina, Popradská kotlina basin, 6787 .

50. Dolina Bielych plies valley, Belianske Tatry Mts. NP, 6787.

51. Dolný Harmanec, Starohorské vrchy Mts., 7180.

52. Dolný Jelenec, Starohorské vrchy Mts., 7180.

53. Dolný Smokovec, swamps, Popradská kotlina basin, 6887.

54. Donovaly, Vel'ká Fatra Mts., 7181.

55. Dubová nr. Stoličný potok brook, Trnavská pahorkatina hills, 7670.

56. Dúbrava, Liptovský Mikuláš distr., Liptovská kotlina basin, 6983.

57. Dúbravka, brook, Malé Karpaty Mts., 7868.

58. Dudváh, Trnavská pahorkatina hills, 7372.

59. Dulova Ves nr. Prešov, wet meadow, Košická kotlina basin, 7093.

60. Dunaj river basin*.

61. Eurocamp FICC env. Tatranská Lomnica, brook, Popradská kotlina basin, 6787.

62. Fialkový potok brook, Malé Karpaty Mts., 7868.

63. Furmanec river, left tributary, S of Kášter, Muránska planina Mts. NP, 7385.

64. Gader river, Turčianska kotlina basin, 7079.

65. Gerlachov env. Bardejov, river, Ondavská vrchovina hills, 6692.

66. Gerlachov, Poprad distr., Popradská kotlina basin, 6887.

67. Gidra stream, Malé Karpaty Mts., 7669.

68. Grúnik, stream, Vysoké Tatry Mts. NP, 6885.

69. Haliar elevation point, Staré Hory env., Starohorské vrchy Mts., 7180.

70. Hankovce, brook, Laborecká vrchovina hills, 6997.

71. Havešová, Bukovské vrchy Mts. NP, 6800.

72. Havraník, Muránska planina Mts. NP, 7186.

73. Herl’any, stream, Košická kotlina basin, 7294.

74. Hiadel'ská dolina valley, Hiadel' env., Nízke Tatry Mts. NP, 7181.

75. Hiadel'ské sedlo saddleback, Korytnica kúpele env., Nízke Tatry Mts. NP, 7181.

76. Hluboký potok brook, Bukovské vrchy Mts. NP, 6900.

77. Holica env. Javorina, brook, Vysoké Tatry Mts. NP, 6786.

78. Horáreň Sliače, Tríbeč hill, 7476 .

79. Horný Smokovec, Popradská kotlina basin, 6887.

80. Hornád river basin*.

81. Horné Lazy, Horehronské podolie basin, 7183.

82. Host'ovce, Košická kotlina basin 7491.

83. Hradište, Hiadel'ská dolina valley, Starohorské vrchy Mts., 7181.

84. Hrdzavá, spring area, Muránska planina Mts. NP, 7285.

85. Hrhov, Slovenský kras karst, 7390.

86. Hriňová, Veporské vrchy Mts., 7383.

87. Hrochotská dolina valley, Hrochot' env., Zvolenská kotlina basin, 7381.

88. Hromacký vrch hill env. Livov, pasture, Čergov Mts., 6792.

89. Hron river basin*.

90. Hrončecký grúň slope, Pol’ana Mts., 7383.

91. Hrušov, Tríbeč hill, 7576 (Fig. 1).

92. Hrušovo, Revúcka vrchovina Mts., 7468.

93. Hučava stream, Pol'ana Mts., 7382.

94. Il’anovo, Liptovská kotlina basin, 6983. 
95. Il’anovská dolina valley, Nízke Tatry Mts. NP, 6983.

96. Ipel' nr. Šahy, Ipel'ská pahorkatina hills, 7979.

97. Jakubany, Spišsko-šarišské medzihorie Imts., 6790.

98. Jamy nr. Tatranská Lomnica, Vysoké Tatry Mts. NP, 6886.

99. Jarabá skala rock, Bukovské vrchy Mts. NP, 6800.

100. Jasná, Nízke Tatry Mts. NP, 7083.

101. Javorina, Podtatranská brázda furrow, 6786.

102. Javorov potok brook, Červené vrchy Mts., Západné Tatry Mts. NP, 6785.

103. Jurkova Vol’a, wet gutter, Ondavská vrchovina hills, 6695.

104. Juskova Vol'a, brook, Slanské vrchy Mts., 7195.

105. Kamenný potok brook, Malé Karpaty Mts., 7669.

106. Kamienka, brook, Beskydské predhorie Mts., 7098.

107. Kapušianske Kl'ačany, swamps, Východoslovenská rovina plain, 7498.

108. Kardolina, Popradská kotlina basin, 6787.

109. Kavečany, brook, Čierna hora Mt., 7293.

110. Kežmarská Biela Voda, Podtatranská kotlina basin, 6888.

111. Kežmarské Žl’aby, Popradská kotlina basin, 6787.

112. Klačianska dolina valley env. Ružomberok, Nízke Tatry Mts. NP, 6982.

113. Klačno, Hornonitrianska kotlina basin, 7077.

114. Kochul'a env. Hiadel', Nízke Tatry Mts. NP, 7181.

115. Kokošovce, brook, Košická kotlina basin, 7094.

116. Kolbasov, stream, Bukovské vrchy Mts. NP, 6900.

117. Korejovce, brook, Laborecká vrchovina hills, 6695.

118. Korytnica, Vel'ká Fatra Mts. NP, 7081.

119. Koš wetlands, Hornonitrianska kotlina basin, 7277 (Fig. 2).

120. Košiar env. Javorina, Belanské Tatry Mts. NP, 6786.

121. Košiar env. Ždiar, Belanské Tatry Mts. NP, 6787.

122. Košice, Košická kotlina basin, 7293.

123. Košická Belá, Košice env., Čierna hora Mt., 7192.

124. Košický Klečenov, brook, Košická kotlina basin, 7295.

125. Krajné Čierno, stream, Ondavská vrchovina hills, 6695.

126. Král'ovský Chlmec, Východoslovenská rovina flat, 7597.

127. Krámec nr. Klačianská dolina valley, Nízke Tatry Mts. NP, 7082.

128. Krásny Brod env. Medzilaborce, spring area, brook, Laborecká vrchovina hills, 6797.

129. Kremnica, Kremnické vrchy Mts., 7279.

130. Kropácová nr. Horná Lehota, Horehronské podolie basin, 7183.

131. Kružlová, well, brook, Ondavská vrchovina hills, 6695.

132. Kružlovská Huta, Ondavská vrchovina hills, 6692.

133. Kvačianka, Chočské vrchy Mts., 6883.

134. Kyjov, Čergov Mts., 7196.

135. Kysak, spring area, Čierna hora Mt., 7193.

136. Kyslinky, Pol'ana Mts., 7382.

137. Latorica river basin*.

138. Leles, swamps, Východoslovenská rovina plain, 7598.

139. Levice, Ipel'ská pahorkatina hills, 7777.

140. Liešt'any, meander, river, Hornonitrianska kotlina basin, 7176.

141. Liptovská Lúžna, brook, Nízke Tatry Mts. NP, 7081.

142. Liptovská Porúbka, Liptovská kotlina basin, 6984.

143. Liptovská Teplička, Nízke Tatry Mts. NP, 7086.

144. Liptovské Klačany, Liptovská kotlina basin, 6982.

145. Liptovské Revúce, Vel'ká Fatra Mts. NP, 7080.

146. Liptovský Hrádok, Liptovská kotlina basin, 6984.

147. Litvorový Žlab, Bielovodská dolina valley, Vysoké Tatry Mts. NP, 6786.

148. Lomnistá dolina valley nr. Brezno, Nízke Tatry Mts. NP, 7182.

149. lower Váh river basin*.

150. Lubel’a, Liptovská kotlina basin, 6982.

151. L'ubochnianska dolina valley, Vel'ká Fatra Mts. NP, 6980.

152. Lúčina, Slanské vrchy Mts., 7194.

153. Lučivná - Sviniarka, spring area, brook, Popradská kotlina basin, 6986.

154. Ludrová, Liptovská kotlina basin, 6982 
155. Lúka, Považský Inovec Mts., 7373.

156. Lukovský vrch hill nr. Ivanovce, Považské podolie basin, 7173.

157. Lupčianska dolina valley nr. Hlinistá, Nízke Tatry Mts. NP, 7082.

158. Lysá Pol'ana env. Žabí potok brook, Vysoké Tatry Mts. NP, 6786.

159. Lysá, Čergov Mts., 6892.

160. Majdan, brook, wet meadow, Čergov Mts., 6892.

161. Makovce, brook, Ondavská vrchovina hills, 6796.

162. Malá Homôlka well, Vtáčnik Mts., 7377.

163. Malá Lodina, Margecany env. Čierna hora Mts., 7192.

164. Malá Pol’ana, spring area, brook, Laborecká vrchovina hills, 6796.

165. Malá Studená dolina valley, Vysoké Tatry Mts. NP, 6886.

166. Malá Svišt'ovka env. Tatranská Lomnica, stream, Vysoké Tatry Mts. NP, 6887.

167. Malacky, Borská nížina lowland, 7568.

168. Malý Studený potok brook nr. Nálepkova chata chalet, Vysoké Tatry Mts. NP, 6887.

169. Margecany brook, Čierna hora Mts., 7192.

170. Marheček, Borská nížina lowlands, 7568 (Fig. 3).

171. Marková dolina valley, Starohorské vrchy Mts., 7181.

172. Martin, Turčianska kotlina basin, 6978.

173. Mat'ovce, Ondavská vrchovina hills, 7498.

174. Meandre, Horehronské podolie basin, 7186.

175. Medvedzie, brook, Laborecká vrchovina hills, 6595.

176. Megova dolina 1 valley, brook, Vtáčnik Mts., 7377.

177. Megova dolina 2 valley, brook, Vtáčnik Mts., 7437.

178. Mengusovce, brook, Vysoké Tatry Mts. NP, 6986.

179. Michal na Ostrave, Podunajská rovina flat, 7971.

180. Michal'any, wet meadow, Východoslovenská pahorkatina hills, 7495.

181. Mičová stream, Pol'ana Mts., 7382.

182. Minčol, well, Čergov Mts. 6792.

183. Mlynica, Popradská kotlina basin, 6887.

184. Mokrý potok brook, Malé Karpaty Mts., 7868.

185. Monkova dolina valley, Belianske Tatry Mts. NP, 6787.

186. Morava river basin*.

187. Morava river, Malé Karpaty Mts., 7569.

188. Moštenica, Zvolenská kotlina basin, 7181.

189. Mrázovce, brook, pasture, Ondavská vrchovina hills, 6896.

190. Muránska Lehota, Stolické vrchy Mts., 7286.

191. Muránska planina plain, spring area, Muránska planina Mts. NP, 7285.

192. Muránska Zdychava, brook, Stolické vrchy Mts., 7286.

193. Nad Šenkárkou env. Limbach, Malé Karpaty Mts., 7669.

194. Nitra - Zobor, Tríbeč hill, 7674.

195. Nitra, Nitrianska pahorkatina hills, 7674.

196. Nitrianske Rudno, water reservoir, Hornonitrianska kotlina basin, 7276 .

197. Nižná Čabina, stream, Laborecká vrchovina hills, 6897.

198. Nižná Kamenica, swamps, Košická kotlina basin, 7294.

199. Nižná Myšl'a, Košická kotlina basin, 7399.

200. Nižná Pisaná, brook, Ondavská vrchovina hills, 6695.

201. Nižné Hágy, Popradská kotlina basin, 6886.

202. Nižné Temnosmrečinske pleso mountain lake, Vysoké Tatry Mts. NP, 6886.

203. Nižný Komárnik, Laborecká vrchovina hills, 6696.

204. Nová Baňa, Vtáčnik Mts., 7577.

205. Nová Lehota, Kremnické vrchy Mts., 7378.

206. Nová Lesná brook, Popradská kotlina basin, 6887.

207. Nová Polianka env. Svidník, brook, wet meadow, Ondavská vrchovina hills, 6795.

208. Nová Sedlica env., Poloniny NP, Bukovské vrchy Mts., 6901.

209. Nová Vieska, Podunajská rovina flat, 8177.

210. Oblazy, Chočské vrchy Mts., 6883.

211. Okrúhly chrbát saddle env. Úhorná, Volovské vrchy Mts., 7289.

212. Olavec well, Diviacka Nová Ves env. Strážovské vrchy Mts., 7276 (Fig. 4).

213. Olejníkov, Majdan env., Čergov Mts., 6892.

214. Olejníkov, Čergov Mts., 6892. 
215. Opatovské jazero lake, Považské podolie basin, 7074.

216. Oravská Jasenica, Oravská kotlina basin, 6682.

217. Oravská Polhora env. Borsučie, stream, Podbeskydská brázda furrow, 6482.

218. Oravské Veselé, Podbeskydská brázda, 6582.

219. Oravský Podzámok, Oravská vrchovina Mts., 6783.

220. Osturňa nr. forest lake, Spišská Magura Mts., 6687.

221. Pálenica, Vysoké Tatry Mts. NP, 6885.

222. Patočiny nr. Korytnica kúpele health resort, Vel'ká Fatra Mts. NP, 7081.

223. Pavčina Lehota, swamps, Liptovská kotlina basin, 6983.

224. Piesky nr. Banská Bystrica, Starohorské vrchy Mts., 7180.

225. Pieštany, Trnavská pahorkatina hills, 7472.

226. Píla nr. Červený Kameň, Malé Karpaty Mts., 7669.

227. Pilsko, Oravské Beskydy Mts, 6482.

228. Pivovarský potok brook, Malá Fatra Mts. NP, 6978.

229. Ploštín, Liptovská kotlina basin, 6983.

230. Pod Muráňom env. Javorina, Vysoké Tatry Mts. NP, 6786.

231. Pod Príslopom env. Podspády, Belianske Tatry Mts. NP, 6787.

232. Podbanské, Liptovská kotlina basin, 6885.

233. Podbiel, Oravská vrchovina Mts., 6682.

234. Podspády, Belianske Tatry Mts. NP, 6787.

235. Pohronský Bukovec, wet maedow, stream, Nízke Tatry Mts. NP, 7182.

236. Poprad - Dunajec river basin*.

237. Poráč, Volovské vrchy Mts., 7190.

238. Prešov, Košická kotlina basin, 7093.

239. Prievidza, Hornonitrianska kotlina basin, 7277.

240. Príslop, wet meadow, Veporské vrchy Mts., 7484.

241. Prostredná stream env. Hiadel', Nízke Tatry Mts., 7181.

242. Ptrukša, pasture, Východoslovenská rovina plain, 7598.

243. Púchalky, Nízke Tatry Mts. NP, 7084.

244. Račková dolina valley, Západné Tatry Mts. NP, 6884 (Fig. 9).

245. Rajecká Lesná, Žilinská kotlina basin, 6977.

246. Rakovčík, brook, wet meadow, Ondavská vrchovina hills, 6795.

247. Rakytovo brook env. Sihla, Veporské vrchy Mts., 7384.

248. Rastislavice, Nitrianska pahorkatina hills, 7874.

249. Ráztoka env. Bukovecká dolina valley, Horehronské podolie basin, 7182.

250. Rejdová, pasture, stream, Revúcka vrchovina Mts., 7287.

251. Remetské Hámre, Východoslovenská pahorkatina hills, 7199.

252. Revištské Podhradie, Vtáčnik Mts., 7478.

253. Revúca, Revúcka vrchovina Mts., 7386.

254. Revúčka, Stolické vrchy Mts., 7286.

255. Rimava river nr. Tisovec, Muránska planina Mts. NP, 7285.

256. Rimavská Sobota, Rimavská kotlina basin, 7686.

257. Rožňava, swamps, Rožňavská kotlina basin, 7389.

258. Rozpadliny env. Bielovodska dolina valley, Vysoké Tatry Mts. NP, 6786.

259. Rudňany, stream, swamps, Volovské vrchy Mts., 7190.

260. Ruská Nová Ves, brook, Košická kotlina basin, 7093.

261. Ružomberok, Liptovská kotlina basin, 6981.

262. Sabinov, brook, Spišsko-šarišské medzihorie Imts., 6892.

263. Šarišské Čierne, wet gutter, Ondavská vrchovina hills, 6694.

264. Sečovce env. Trebišov, Východoslovenská rovina flat, 7295.

265. Senné, Krupinská planina Mts., 7682.

266. Šišulákov mlyn mill, Borská nížina lowland, 7468.

267. Skalnatý potok brook env. Tatranská Lomnica, Popradská kotlina basin, 6787.

268. Škaredý žl'ab trough env. Kôprová dolina valley, Vysoké Tatry Mts. NP, 6885.

269. Slaná river basin*.

270. Slančík, Východoslovenská pahorkatina hills, 7395.

271. Slanská dolina valley env. Rejdová, brook, Stolické vrchy Mts., 7287.

272. Smrekovec, Vysoké Tatry Mts. NP, 6886.

273. Soblahov, Strážovské vrchy Mts., 7174.

274. Sol', brook, Východoslovenská pahorkatina hills, 7095. 
275. Solivar env. Prešov, swamps, Košická kotlina basin, 7093.

276. Sol'ný potok brook, Oravské Beskydy Mts., 6483.

277. Somotor, Východoslovenská rovina flat, 7596.

278. Sopotnická dolina valley, Brusno env., Horehronské podolie basin, 7182.

279. Spády waterfall, Pol'ana Mts., 7383.

280. Špania Dolina, Starohorské vrchy Mts., 7180.

281. Stakčín, wet meadow, Laborecká vrchovina hills, 6999.

282. Stanišovská dolina valley env. Liptovský Mikuláš, Nízke Tatry Mts. NP, 6984.

283. Stanišovské sedlo saddleback nr. Liptovská Porúbka, Nízke Tatry Mts. NP, 6984.

284. Stará L’ubovňa, spring area, Spišsko-šarišské medzihorie Imts., 6690.

285. Stará Pila, brook, Starohorské vrchy Mts., 7180.

286. Stará Voda, Volovské vrchy Mts. 7290.

287. Staré Hory, Starohorské vrchy Mts., 7180.

288. Starina env., brook, Laborecká vrchovina hills, 6899.

289. Štart nr. Tatranská Lomnica, Vysoké Tatry Mts. NP, 6886.

290. Stavanie, Strážovské vrchy Mts., 7176.

291. Stinská slatinka meadow, Bukovské vrchy Mts. NP, 6901.

292. Štôla, Popradská kotlina basin, 6986.

293. Stratená jaskyňa cave, Slovenský raj NP, 7195.

294. Štrbské pleso mountain lake, Popradská kotlina basin, 6886.

295. Štrbské Pleso, Popradská kotlina basin, 6886.

296. Streda nad Bodrogom, Východoslovenská rovina plain, 7696.

297. Strieborný potok brook nr. Rimava, Veporské vrchy Mts., 7285.

298. Strmý vrštek hill nr. Dolná Lehota, Horehronské podolie flat, 7183.

299. Studenovodské vodopády waterfalls, stream, Vysoké Tatry Mts. NP, 6887.

300. Stupavský potok brook, Malé Karpaty Mts., 7668.

301. Stužica, Nová Sedlica, Poloniny NP, Bukovské vrchy Mts., 6901.

302. Stužická rieka stream, Poloniny NP, Bukovské vrchy Mts., 6901.

303. Súčanka brook nr. Dolna Suča, Biele Karpaty Mts., 7074.

304. Sučany, Turčianska kotlina basin, 6879.

305. Suchá dolina valley, Tatranska Kotlina env., Popradská kotlina basin, 6787.

306. Suchý vrch hill env. Javorina, brook, Vysoké Tatry Mts. NP, 6886.

307. Šuja, Žilinská kotlina basin, 6977.

308. Súl'ov, Strážovské vrchy Mts., 6877.

309. Š́r, swamps, Podunajská rovina flat, 7769.

310. Svätý Kríž, Liptovská kotlina basin, 6983.

311. Sveržov, wet gutter, Ondavská vrchovina hills, 6692.

312. Svidnícke sedlo saddleback env. Vyšný Komárnik, brook, Laborecká vrchovina hills, 6596.

313. Svidník, stream, Ondavská vrchovina hills, 6695.

314. Svišt'ovský potok brook, Vysoké Tatry Mts. NP, 6886.

315. Svit, Popradská kotlina basin, 6987.

316. Tále env. Brezno, Horehronské podolie basin, 7183.

317. Tatranská Kotlina, swamps, Popradská kotlina basin, 6787.

318. Tatranská Lesná, brook, wet meadow, Popradská kotlina basin, 6887.

319. Tatranská Lomnica, Popradská kotlina basin, 6787.

320. Tatranská Polianka, Popradská kotlina basin, 6887.

321. Tatranské Matliare env. Poprad, Popradská kotlina basin, 6887.

322. Teriakovce, brook, Košická kotlina basin, 7093.

323. Tichá dolina valley env. Podbanské, brook, Liptovská kotlina basin, 6885.

324. Tisovec, Muránska planina Mts. NP, 7385.

325. Tokáreň, spring area, brook, Čergov Mts., 6892.

326. Tokárensky potok brook nr. Ždiar, Belianske Tatry Mts. NP, 6787.

327. Tomanová dolina valley, Západné Tatry Mts. NP, 6785.

328. Topol'a, river, Bukovské vrchy Mts. NP, 6900.

329. Trebatice, Trnavská pahorkatina hills, 7472.

330. Trenčín, Považské podolie basin, 7174.

331. Tri Studničky env. Pavučia dolina valley, brook, Liptovská kotlina basin, 6885.

332. Tri studničky well nr. Podbanské, brook, Liptovská kotlina basin, 6885.

333. Tristárska dolina valley env. Ždiar, stream, Belianske Tatry Mts. NP, 6787.

334. Trstenec well env. Diviacka Nová Ves, Strážovské vrchy Mts., 7276 (Fig. 6). 
335. Tuhrina, swamps, Slanské vrchy Mts., 7194.

336. Turiec river basin, Turčianska kotlina basin, 7079.

337. Tužina, Strážovské vrchy Mts., 7077.

338. Ubl'a, Beskydské predhorie Mts., 7100.

339. Ubl'ianka, Bukovské vrchy Mts. NP, 7099.

340. Úhorná, swamps, Volovské vrchy Mts., 7290.

341. Ul'anka nr. Suchý vrch hill, Starohorské vrchy Mts., 7180.

342. Ulič, pasture, stream, Bukovské vrchy Mts. NP, 7000.

343. Uličské Krivé, swamps, Bukovské vrchy Mts. NP, 7000.

344. Úplaz env. Javorová dolina valley, Vysoké Tatry Mts. NP, 6786.

345. upper Váh river basin*.

346. Utekáč, Stolické vrchy Mts., 7384.

347. Valaská Dubová, spring area, Chočské vrchy Mts., 6881.

348. Valča river, Turčianska kotlina basin, 6978.

349. Val'kovňa, Horehronské podolie basin, 7186.

350. Vážna nr. Lučatín, brook, Zvolenská kotlina basin, 7281.

351. Velčice, Tríbeč hill, 7575.

352. Vel'ká Homôlka well, Vtáčnik Mts., 7377.

353. Vel'ká Pálenica, Vysoké Tatry Mts. NP, 6885.

354. Vel'ká Svištovka, Vysoké Tatry Mts. NP, 6787.

355. Vel'kolúcky potok brook, Malé Karpaty Mts., 7868.

356. Vel'ký Brankov, brook, Nízke Tatry Mts. NP, 7081.

357. Vel'ký Dvor, Slovenský raj NP, 7191.

358. Vel'ký Horeš, swamps, Východoslovenská rovina plain, 7697.

359. Vel'ký Sokol, Slovenský raj NP, 7095.

360. Vernár, Slovenský raj NP, 7187.

361. Vihorlat, Vihorlat Mts., 7198.

362. Viničky, Zemplínske vrchy Mts., 7596.

363. Vlčia jaskyňa cave, Slovenský raj NP, 7187.

364. Vlkolínec, Vel'ká Fatra Mts. NP, 6981.

365. Vojenská jaskyňa cave, Slovenský raj NP, 7187.

366. Vojtasova pol'ana meadow, Belianske Tatry Mts. NP, 6787.

367. Volica, pasture, Laborecká vrchovina hills, 6897.

368. Vráble, Hronská pahorkatina hills, 7775.

369. Vranov nad Topl'ou, stream, Východoslovenská pahorkatina hills, 7196.

370. Vrátna dolina valley, Malá Fatra Mts. NP, 7080.

371. Vrbany env. Diviacka Nová Ves, river, Hornonitrianska kotlina basin, 7277.

372. Východná, Liptovská kotlina basin, 6985.

373. Vydrica stream nr. Kačín, Malé Karpaty Mts., 7768.

374. Vyšná Kamenica, stream, Košická kotlina basin, 7294.

375. Vyšná Maša, Volovské vrchy Mts., 7187.

376. Vyšné Hágy, Popradská kotlina basin, 6886.

377. Vyšné Račkové pleso mountain lake, Západné Tatry Mts. NP, 6884.

378. Vyšné Temnosmrečinske pleso mountain lake, Vysoké Tatry Mts. NP, 6886.

379. Vysoké Tatry Mts. NP *.

380. Zádiel, Slovenský kras karst NP, 7391.

381. Zadná Kopa env. Javorina, brook, Vysoké Tatry Mts. NP, 6786.

382. Zadná Pol'ana, Pol'ana Mts., 7382.

383. Zadné Med'odoly, wet meadow, Vysoké Tatry Mts. NP, 6786.

384. Zámutov env. Vranov nad Topl'ou, brook, Východoslovenská pahorkatina hills, 7095

385. Žarnovica, Žiarska kotlina basin, 7578.

386. Zboj, stream, Bukovské vrchy Mts. NP, 6900.

387. Zbojnícka jaskyňa cave, Malé Karpaty Mts., 7768.

388. Zbojská env. Fabová hol’a Mt., Veporské vrchy Mts., 7285.

389. Ždiar nr. Magura, Podtatranská brázda furrow, 6787.

390. Železná Breznica, brook, Kremnické vrchy Mts., 7380 (Fig. 11).

391. Železná studnička well, Malé Karpaty Mts., 7768.

392. Zemplínske Hámre, brook, Vihorlatské vrchy Mts., 7098.

393. Zimovci, Myjavská pahorkatina hills, 7171.

394. Žitná - Radiša nr. Karolintál, Nitrianska pahorkatina hills, 7276. 
395. Zlatá Baňa env. Prešov, Slanské vrchy Mts., 7094.

396. Zvolen, Zvolenská kotlina basin, 7480.

*Note: Bulánková (2003) and Enderlein (1937) published localities, which are difficult to manage in geomorphological units and grid mapping codes of DFS (Bulánková studied water courses, that pass through many grid codes and Enderlein published only the name of the mountain), therefore, collecting sites are reported without this supplement data.

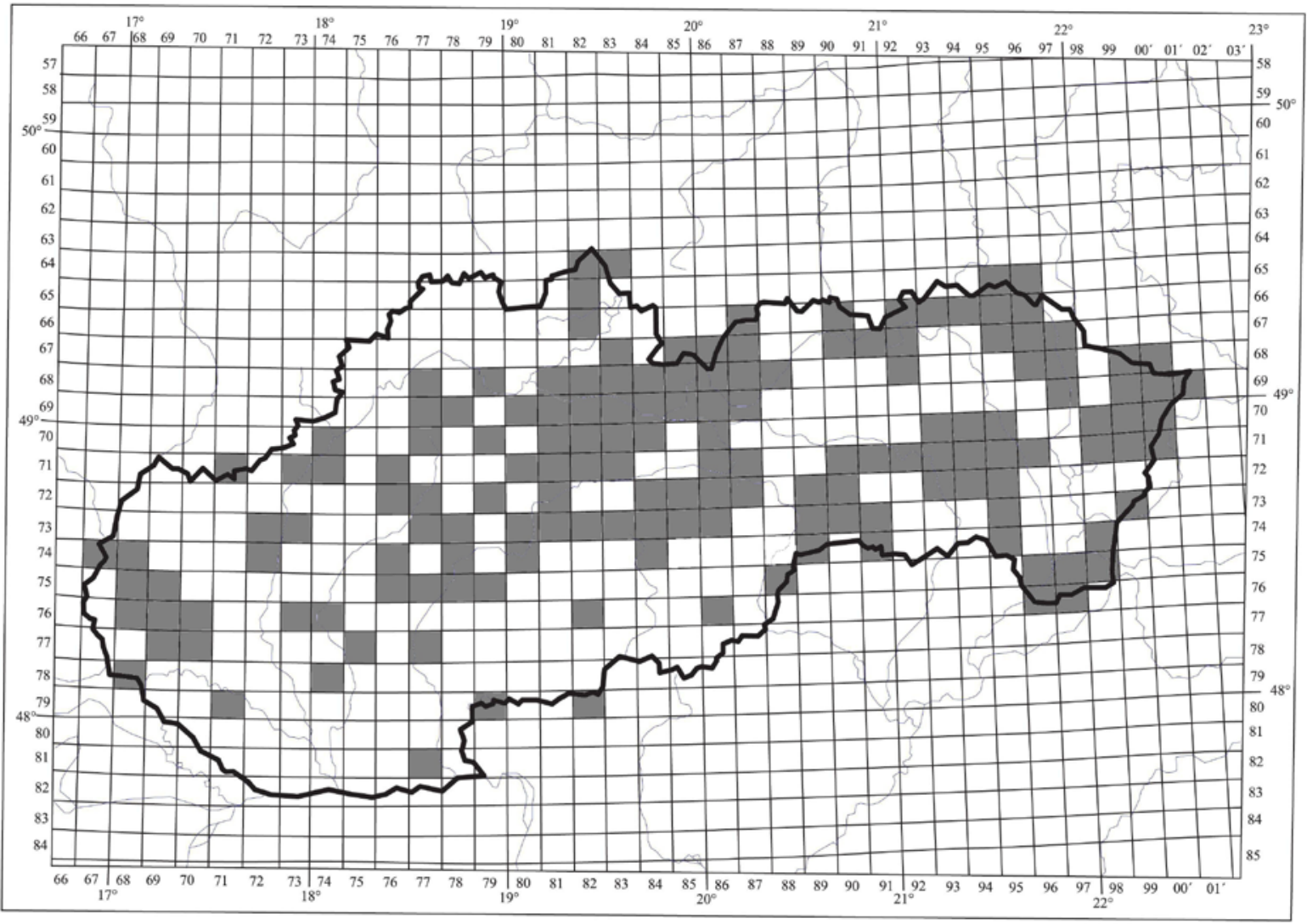

Map 1: Map showing all records of moth flies from Slovakia

(DFS: 6482 - Oravská Polhora, Pilsko, 6483 - Babia hora Mt., Sol’ný potok brook, 6582 - Oravské Veselé, 6595 - Medvedzie, 6596 Svidnícke sedlo saddleback, 6682 - Biela Orava river, Oravská Jasenica, Podbiel, 6687 - Osturňa, 6690 - Stará Luubovňa, 6692 - Gerlachov, Kružlovská Huta, Sveržov, 6693 - Bardejov, Bardejovské Kúpele, 6694 - Šarišské Čierne, 6695 - Jurkova Vol’a, Korejovce, Krajné Čierno, Kružlová, Nižná Pisaná, Svidník, 6696 - Nižný Komárnik, 6783 - Oravský Podzámok, 6785 - Javorov potok brook, Tomanová dolina valley, 6786 - Holica, Javorina, Košiar, Litvorový Žlab, Lysá Pol’ana, Pod Muráňom, Rozpadliny, Úplaz, Zadná Kopa, Zadné Med’odoly, 6787 - Alabastrová jaskyňa cave, Babí potok brook, Biela, Čierna dolina valley, Dolina 7 prameňov valley, Dolina Bielych ples valley, Eurocamp FICC, Kardolina, Kežmarské Žlaby, Košiar, Monkova dolina valley, Pod Príslopom, Podspády, Skalnatý potok brook, Suchá dolina valley, Tatranská Kotlina, Tatranská Lomnica, Tokárensky potok brook, Tristárska dolina valley, Vel'ká Svištoovka, Vojtasova pol’ana meadow, Ždiar, 6790 - Jakubany, 6791 - Č́ŕč, 6792 - Hromacký vrch hill, Minčol, 6795 - Nová Polianka, Rakovčík, 6796 - Bukovce, Makovce, Malá Pol’ana, 6797 - Krásny Brod, 6800 - Havešová, Jarabá skala rock, 6877 - Súl’ov, 6879 - Sučany, 6881 - Valaská Dubová, 6882 - Vel'ký Choč, 6883 - Kvačianka, Oblazy, 6884 - Račková dolina valley, Vyšné Račkové pleso mountain lake, 6885 - Grúnik, Pálenica, Podbanské, Škaredý žlab trough, Tichá dolina valley, Tri Studničky, Vel'ká Pálenica, 6886 - Biely Váh river, Danielov dom house, Jamy, Malá Studená dolina valley, Nižné Hágy, Nižné Temnosmrečinske pleso mountain lake, Smrekovec, Štart, Štrbské pleso mountain lake, Suchý vrch hill, Svištovský potok brook, Vyšné Hágy, Vyšné Temnosmrečinske pleso mountain lake, 6887 - Červený potok brook, Dolný Smokovec, Gerlachov, Malá Svištovka, Malý Studený potok brook, Mlynica, Nová Lesná, Studenovodské vodopády waterfalls, Tatranská Lesná, Tatranská Polianka, Tatranské Matliare, 6888 - Kežmarská Biela Voda stream, 6892 - Baranie, Lysá, Majdan, Olejníkov, Sabinov, Tokáreň, 6896 - Mrázovce, 6897 - Nižná Čabina, 6899 - Starina, 6900 - Hluboký potok brook, Kolbasov, Topola, Zboj, 6901 Nová Sedlica, Stinská slatinka meadow, Stužica, Stužická rieka stream, 6977 - Rajecká Lesná, Šuja, 6978 - Martin, Pivovarský potok brook, Valča, 6980 - Lubochnianska dolina valley, 6981 - Bukovinka, Ružomberok, Vlkolinec, 6982 - Klačianska dolina valley, Liptovské Klačany, Lubel'a, Ludrová, 6983 - Demänovská dolina valley, Dúbrava, Il’anovo, Ilanovská dolina valley, Pavčina Lehota, Ploštín, Svätý Kríž, 6984 - Brtkovica, Liptovská Porúbka, Liptovský Hrádok, Stanišovská dolina valley, Stanišovské sedlo saddleback, 6985 - Východná, 6986 - Lučivná - Sviniarka, Mengusovce, Stôla, 6987 - Batizovce, Svit, 6997 - Dedačov, Hankovce, 6999 - Stakčín, 7000 - Ulič, Uličské Krivé, 7074 - Súčanka, 7077 - Klačno, Tužina, 7079 - Gader, Turiec, 7080 - Liptovské Revúce, Vrátna dolina valley, 7081 - Bansko, Červená Magura, Korytnica, Liptovská Lúžna, Patočiny, Velký Brankov, 7082 - Krámec, Lupčianska dolina valley, 7083 - Jasná, 7084 Púchalky, 7086 - Liptovská Teplička, 7093 - Dulova Ves, Prešov, Ruská Nová Ves, Solivar, Teriakovce, 7094 - Kokošovce, Zlatá Baňa, 7095 - Čaklov, Sol', Vel'ký Sokol, Zámutov, 7098 - Belá nad Cirochou, Kamienka, Zemplínske Hámre, 7099 - Ublianka, 7100 - Ubla, 7171 - Zimovci, 7173 - Lukovský vrch hill, 7174 - Soblahov, Trenčín, 7176 - Čierna Lehota, Liešt’any, Stavanie, 7180 - Dolný Harmanec, Dolný Jelenec, Haliar, Hiadel'ská dolina valley, Piesky, Špania Dolina, Staré Hory, Stará Pila, Ulanka, 7181 - Baláže, Bukovec, Donovaly, Hiadelské sedlo saddleback, Hradište, Kochula, Marková dolina valley, Moštenica, Prostredná, 7182 - Bukovecká dolina valley, Čierny 
Diel, Lomnistá dolina valley, Pohronský Bukovec, Ráztoka, Sopotnická dolina valley, 7183 - Brezno, Bystrá, Čierny Hron river, Črmné, Horné Lazy, Kropácová, Strmý vrštek hill, Tále, 7186 - Havraník, Meandre, Val'kovňa, 7187 - Dobšinská l'adová jaskyňa cave, Vernár, Vlčia jaskyňa cave, Vojenská jaskyňa cave, Vyšná Maša, 7190 - Poráč, Rudňany, 7191 Vel'ký Dvor, 7192 - Košická Belá, Malá Lodina, Margecany, 7193 - Kysak, 7194 - Červenica, Lúčina, Tuhrina, 7195 - Banské, Juskova Vola, Stratená jaskyňa cave, 7196 - Kyjov, Vranov nad Toplou, 7198 - Vihorlat, 7199 - Remetské Hámre, 7276 - Diviacka Nová Ves, Nitrianske Rudno, Olavec, Trstenec, Žitná - Radiša, 7277 - Koš, Prievidza, Vrbany, 7279 - Kremnica, Turiec, 7281 - Vážna, 7284 - Antalova studnička well, Bánovo, Dlhý Grúň, 7285 Hrdzavá, Muránska planina plain, Rimava, Strieborný potok brook, Zbojská, 7286 - Muránska Lehota, Muránska Zdychava, Revúčka, 7287 - Čierna Lehota, Rejdová, Slanská dolina valley, 7289 - Okrúhly chrbát ridge, 7290 - Stará Voda, Úhorná, 7293 - Čermel', Kavečany, Košice, 7294 - Herl’any, Nižná Kamenica, 7295 - Košický Klečenov, Sečovce, 7372 - Dudváh, 7373 - Lúka, 7377 - Malá Homôlka, Megova dolina valley, Vel'ká Homôlka, 7378 - Nová Lehota, 7380 - Železná Breznica, 7381 - Hrochotská dolina valley, 7382 - Hučava, Kyslinky, Mičová, Zadná Pol’ana, 7383 - Hriňová, Hrončecký grúň slope, Spády, 7384 - Čierny Hron river, Rakytovo, Utekáč, 7385 Furmanec river, Tisovec, 7386 - Revúca, 7389 - Rožňava, 7390 - Hrhov, 7391 - Zádiel, 7395 - Slančík, 7399 - Nižná Myšla, 7467 Megova dolina valley, 7468 - Šišulákov mlyn mill, 7472 - Piešt’any, Trebatice, 7476 - Horáreň Sliače, 7478 - Revišské Podhradie, 7480 Zvolen, 7484 - Príslop, 7489 - Čičarovce, 7491 - Host’ovce, 7495 - Michal'any, 7498 - Kapušianske Kl'ačany, Mat’ovce, 7568 - Malacky, Marheček, 7569 - Morava, 7575 - Velčice, 7576 - Hrušov, 7577 - Brehy, Nová Baňa, 7578 - Žarnovica, 7588 - Bretka, 7596 - Somotor, Viničky, 7597 - Král'ovský Chlmec, 7598 - Leles, Ptrukša, 7668 - Stupavský potok brook, 7669 - Gidra, Kamenný potok brook, Nad Šenkárkou, Píla, 7670 - Dubová, 7673 - Bábsky les forest, 7674 - Nitra - Zobor, Nitra, 7682 - Senné, 7686 - Rimavská Sobota, 7696 Streda nad Bodrogom, 7697 - Vel'ký Horeš, 7768 - Vydrica, Zbojnícka jaskyňa cave, Železná studnička well, 7769 - Šúr, 7775 - Vráble, 7777 - Levice, 7868 - Bratislava, Dúbravka, Fialkový potok brook, Mokrý potok brook, Vel'kolúcky potok brook, 7874 - Rastislavice, 7971 - Michal na Ostrave, 7979 - Ipel', 7982 - Brusno, 8177 - Nová Vieska).

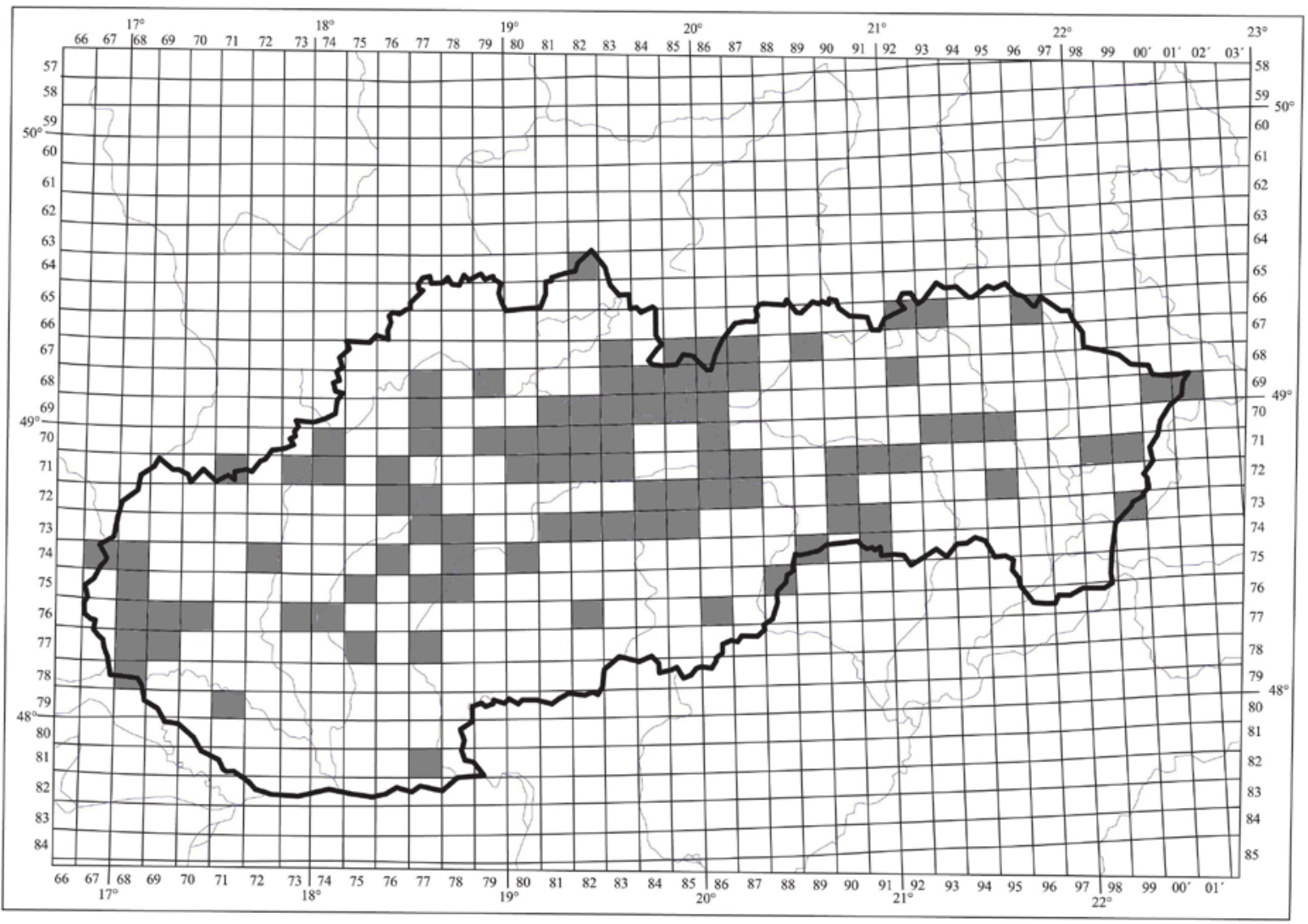

Map 2: Map showing literary records of moth flies from Slovakia

(DFS: 6482 - Pilsko, 6692 - Gerlachov, Kružlovská Huta, 6693 - Bardejov, 6696 - Nižný Komárnik, 6783 - Oravský Podzámok, 6785 Javorov potok brook, 6786 - Javorina, Košiar, Litvorový Žlab, Pod Muráňom, Rozpadliny, Úplaz, Zadná Kopa, 6787 - Babí potok brook, Čierna dolina valley, Dolina 7 prameňov valley, Dolina Bielych ples valley, Kardolina, Kežmarské Žlaby, Košiar, Monkova dolina valley, Pod Príslopom, Podspády, Suchá dolina valley, Tatranská Kotlina, Tatranská Lomnica, Tokárensky potok brook, Vojtasova pol’ana meadow, Ždiar, 6790 - Jakubany, 6877 - Súlov, 6879 - Sučany, 6884 - Račková dolina valley, Vyšné Račkové pleso mountain lake, 6885 Podbanské, Tichá dolina valley, Tri studničky well, 6886 - Danielov dom house, Jamy, Nižné Hágy, Nižné Temnosmrečinske pleso mountain lake, Smrekovec, Štart, Štrbské pleso mountain lake, Suchý vrch hill, Vyšné Hágy, Vyšné Temnosmrečinske pleso mountain lake, 6887 - Gerlachov, Mlynica, Tatranské Matliare, 6892 - Majdan, 6900 - Hluboký potok brook, 6901 - Nová Sedlica, Stužica, Stužická rieka stream, 6977 - Rajecká Lesná, Šuja, 6978 - Valča, 6981 - Bukovinka, Ružomberok, Vlkolinec, 6982 - Kl’ačianska dolina valley, Liptovské Klačany, Lubela, Ludrová, 6983 - Demänovská dolina valley, Dúbrava, Il’anovo, Il'anovská dolina valley, Pavčina Lehota, Ploštín, Svätý Kríž, 6984 - Brtkovica, Liptovská Porúbka, Liptovský Hrádok, Stanišovská dolina valley, Stanišovské sedlo saddleback, 6985 - Východná, 6986 - Mengusovce, Štôla, 7074 - Súčanka, 7077 - Klačno, Tužina, 7079 - Gader, Turiec, 7080 - Liptovské Revúce, Vrátna dolina valley, 7081 - Červená Magura, Korytnica, Patočiny, 7082 - Krámec, Lupčianska dolina valley, 7083 - Jasná, 7086 - Liptovská Teplička, 7093 Dulova Ves, Solivar, 7094 - Zlatá Baňa, 7095 - Vel'ký Sokol, 7171 - Zimovci, 7173 - Lukovský vrch hill, 7174 - Soblahov, Trenčín, 7176 - Čierna Lehota, Stavanie, 7180 - Dolný Harmanec, Dolný Jelenec, Haliar, Hiadel'ská dolina valley, Piesky, Špania Dolina, Staré Hory, Ul’anka, 7181 - Baláže, Bukovec, Donovaly, Hiadelské sedlo saddleback, Hradište, Kochula, Marková dolina valley, Moštenica, Prostredná, 7182 - Bukovecká dolina valley, Lomnistá dolina valley, Pohronský Bukovec, Ráztoka, Sopotnická dolina valley, 7183 - Brezno, Bystrá, Čierny Hron river, Črmné, Horné Lazy, Kropácová, Strmý vrštek hill, Tále, 7186 - Meandre, Val'kovňa, 7187 - Dobšinská laadová jaskyňa 
cave, Vernár, Vyšná Maša, 7190 - Poráč, 7191 Vel'ký Dvor, 7192 - Košická Belá, Malá Lodina, Margecany, 7198 - Vihorlat, 7199 Remetské Hámre, 7276 - Diviacka Nová Ves, 7276 Žitná - Radiša, 7277 - Vrbany, 7279 - Turiec, 7284 - Antalova studnička well, Bánovo, Dlhý Grúň, 7285 - Muránska planina plain, Rimava, Zbojská , 7286 - Revúčka, 7287 - Čierna Lehota, 7290 - Stará Voda, 7295 - Sečovce, 7372 - Dudváh, 7377 - Malá Homôlka, Megova dolina valley, Vel'ká Homôlka, 7378 - Nová Lehota, 7381 - Hrochotská dolina valley, 7382 - Hučava, Kyslinky, Mičová, Zadná Pol’ana, 7383 - Hriňová, Hrončecký grúň slope, Spády, 7384 - Čierny Hron, Rakytovo, Utekáč, 7385 Furmanec river, Tisovec, 7390 - Hrhov, 7391 - Zádiel, 7399 - Nižná Myšla, 7467 - Megova dolina valley, 7468 - Šišulákov mlyn mill, 7472 - Piešt'any, 7476 - Horáreň Sliače, 7478 - Revišské Podhradie, 7480 - Zvolen, 7489 - Čičarovce, 7491 - Host’ovce, 7568 - Malacky, 7569 - Morava, 7575 - Velčice, 7577 - Brehy, Nová Baňa, 7578 - Žarnovica, 7588 - Bretka, 7668 - Stupavský potok brook, 7669 - Gidra, Kamenný potok brook, Nad Šenkárkou, Píla, 7670 - Dubová, 7673 - Bábsky les forest, 7674 - Nitra - Zobor, Nitra, 7682 - Senné, 7686 Rimavská Sobota, 7768 - Vydrica, Zbojnícka jaskyňa cave, Železná studnička well, 7769 - Šúr, 7775 - Vráble, 7777 - Levice, 7868 Bratislava, Fialkový potok brook, Mokrý potok brook, Vel'kolúcky potok, 7971 - Michal na Ostrave, 8169 - Bukovinka, 8177 - Nová Vieska).

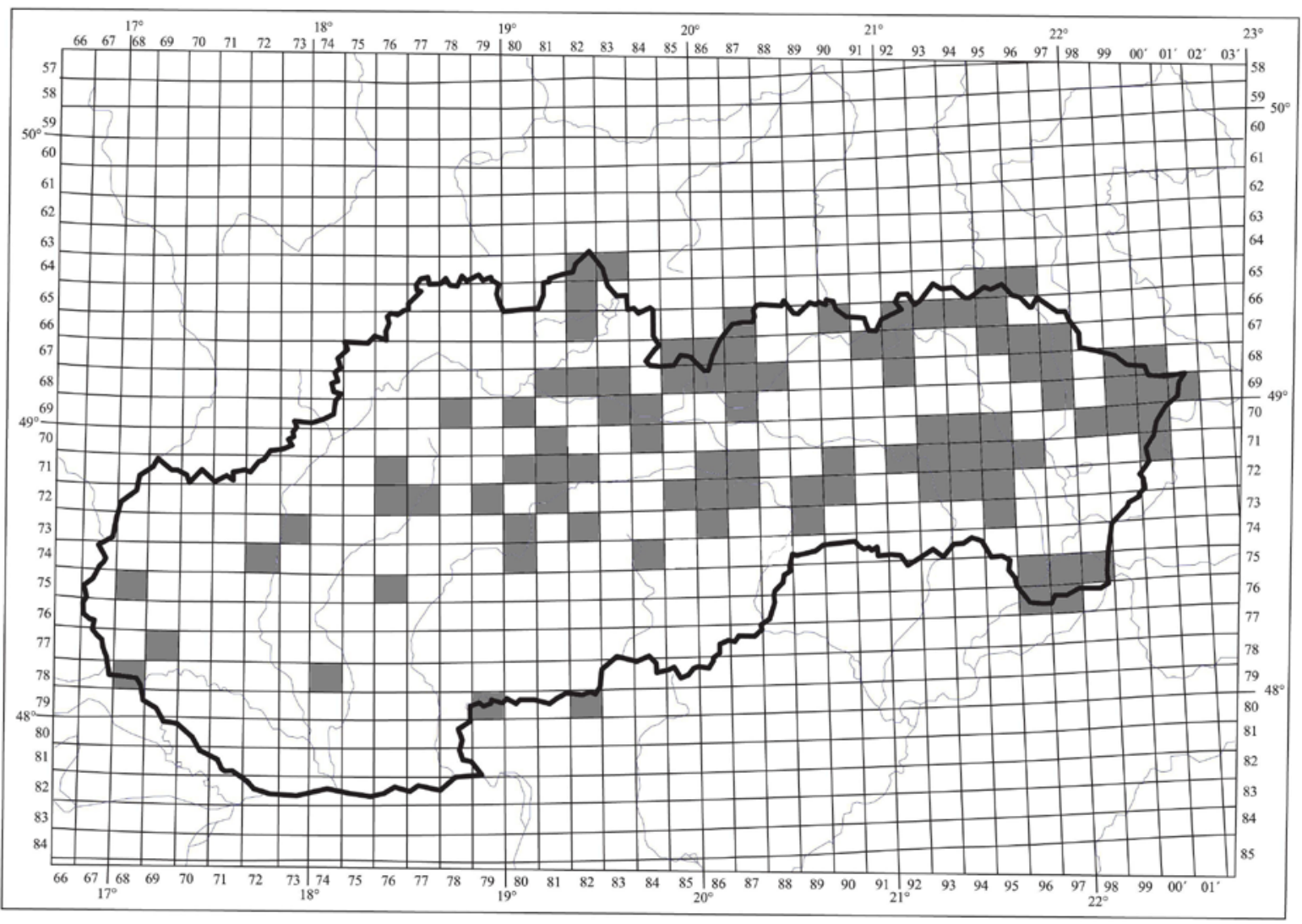

Map 3: Map showing records of new sampled sites of moth flies from Slovakia

(DFS: 6482 - Oravská Polhora, 6483 - Babia hora Mt., Sol’ný potok brook, 6582 - Oravské Veselé, 6595 - Medvedzie, 6596 - Svidnícke sedlo saddleback, 6682 - Biela Orava river, Oravská Jasenica, Podbiel, 6687 - Osturňa, 6690 - Stará Lubovňa, 6692 - Gerlachov, Sveržov, 6693 - Bardejovské Kúpele, 6694 - Šarišské Čierne, 6695 - Jurkova Vol’a, Korejovce, Krajné Čierno, Kružlová, Nižná Pisaná, Svidník, 6785 - Tomanová dolina valley, 6786 - Holica, Lysá Pol’ana, Úplaz, Zadná Kopa, Zadné Med’odoly, 6787 - Alabastrová jaskyňa cave, Biela, Čierna dolina valley, Eurocamp FICC, Skalnatý potok brook, Tatranská Kotlina, Tristárska dolina valley, Vel'ká Svištovka, Ždiar, 6791 - Čírč, 6792 - Hromacký vrch hill, Minčol, 6795 - Nová Polianka, Rakovčík, 6796 - Bukovce, Makovce, Malá Pol'ana, 6797 - Krásny Brod, 6800 - Havešová, Jarabá skala rock, 6881 - Valaská Dubová, 6882 - Vel'ký Choč, 6883 - Kvačianka, Oblazy, 6885 - Grúnik, Pálenica, Škaredý žl'ab trough, Tri Studničky well, Vel'ká Pálenica, 6886 - Biely Váh river, Malá Studená dolina valley, Suchý vrch hill, Svišt'ovský potok brook, 6887 - Červený potok brook, Dolný Smokovec, Malá Svišt'ovka, Malý Studený potok brook, Nová Lesná, Studenovodské vodopády waterfalls, Tatranská Lesná, Tatranská Polianka, 6888 - Kežmarská Biela Voda stream, 6892 - Baranie, Lysá, Majdan, Olejníkov, Sabinov, Tokáreň, 6896 - Mrázovce, 6897 - Nižná Čabina, 6899 - Starina, 6900 - Kolbasov, Topol’a, Zboj, 6901 Nová Sedlica, Stinská slatinka meadow, 6978 Martin, Pivovarský potok brook, 6980 - L'ubochnianska dolina valley, 6983 - Pavčina Lehota, 6984 - Liptovský Hrádok, Stanišovské sedlo saddleback, 6986 - Lučivná - Sviniarka, Mengusovce, 6987 - Batizovce, Svit, 6997 - Dedačov, Hankovce, 6999 - Stakčín, 7000 - Ulič, Uličské Krivé, 7081 - Bansko, Liptovská Lúžna, Vel'ký Brankov, 7084 - Púchalky, 7093 - Dulova Ves, Prešov, Ruská Nová Ves, Solivar, Teriakovce, 7094 - Kokošovce, 7095 - Čaklov, Sol', Zámutov, 7098 - Belá nad Cirochou, Kamienka, Zemplínske Hámre, 7099 - Ubl'ianka, 7100 - Ubl’a, 7176 - Liešt’any, 7180 - Stará Pila, 7181 - Baláže, Donovaly, 7182 - Čierny Diel, Pohronský Bukovec, 7186 - Havraník, 7187 - Vlčia jaskyňa cave, Vojenská jaskyňa cave, 7190 - Rudňany, 7192 - Margecany, 7193 Kysak, 7194 - Červenica, Lúčina, Tuhrina, 7195 - Banské, Juskova Vol’a, Stratená jaskyňa cave, 7196 - Kyjov, Vranov nad Topl'ou, 7276 Diviacka Nová Ves, Nitrianske Rudno, Olavec, Trstenec, 7277 - Koš, Prievidza, Vrbany, 7279 - Kremnica, 7281 - Vážna, 7285 - Hrdzavá, Muránska planina plain, Strieborný potok brook, 7286 - Muránska Lehota, 7286 - Muránska Zdychava, 7287 - Rejdová, Slanská dolina valley, 7289 - Okrúhly chrbát, 7290 - Úhorná, 7293 - Čermel', Kavečany, Košice, 7294 - Herl’any, Nižná Kamenica, 7295 - Košický Klečenov, Sečovce, 7373 - Lúka, 7380 - Železná Breznica, 7382 - Hučava, 7386 - Revúca, 7389 - Rožňava, 7395 - Slančík, 7472 Piešt'any, Trebatice, 7480 - Zvolen, 7484 - Príslop, 7495 - Michal'any, 7498 - Kapušianske Kl'ačany, Mat'ovce, 7568 - Marheček, 7576 Hrušov, 7596 - Somotor, Viničky, 7597 - Král’ovský Chlmec, 7598 - Leles, Ptrukša, 7696 - Streda nad Bodrogom, 7697 - Vel'ký Horeš, 7769 - Š́r, 7868 - Dúbravka 7874 - Rastislavice, 7979 - Ipel', 7982 - Brusno). 


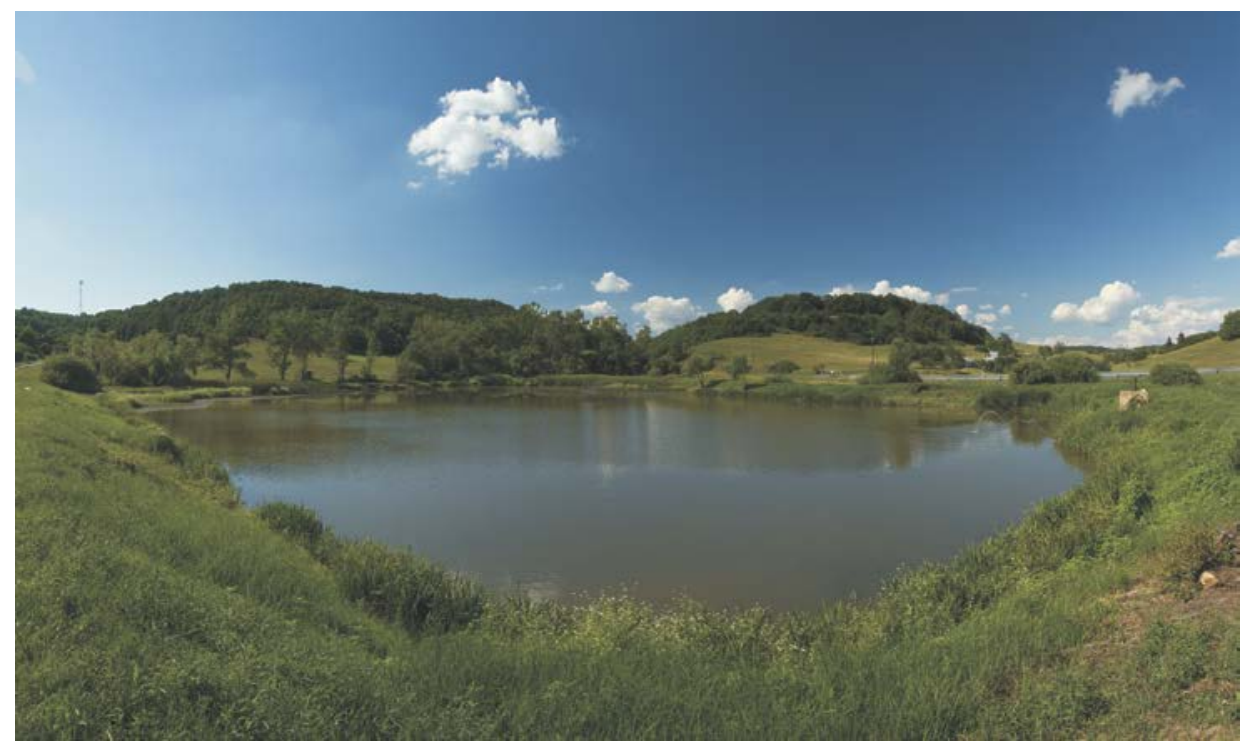

Fig 1: Hrušov, wetlands (photo by Marek Svitok).

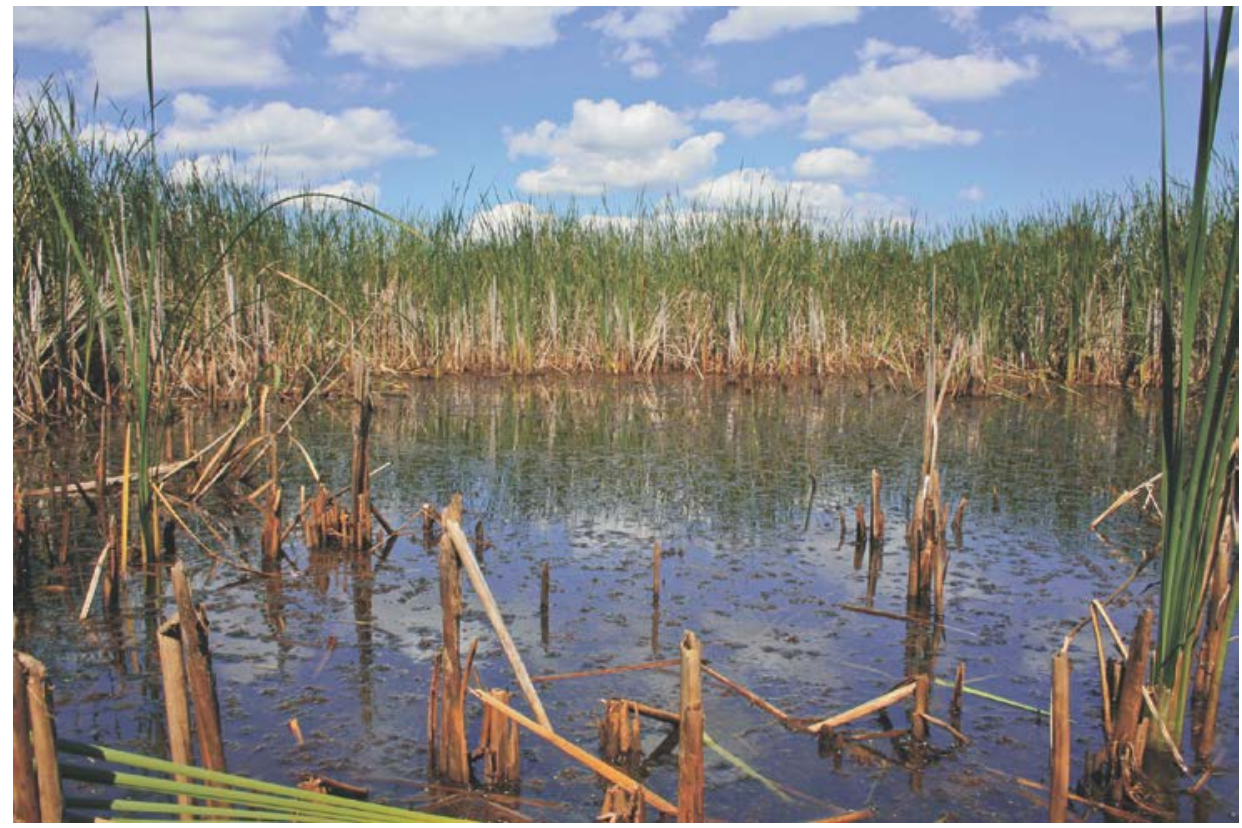

Fig 2: Koš, wetlands (photo by Marek Svitok).

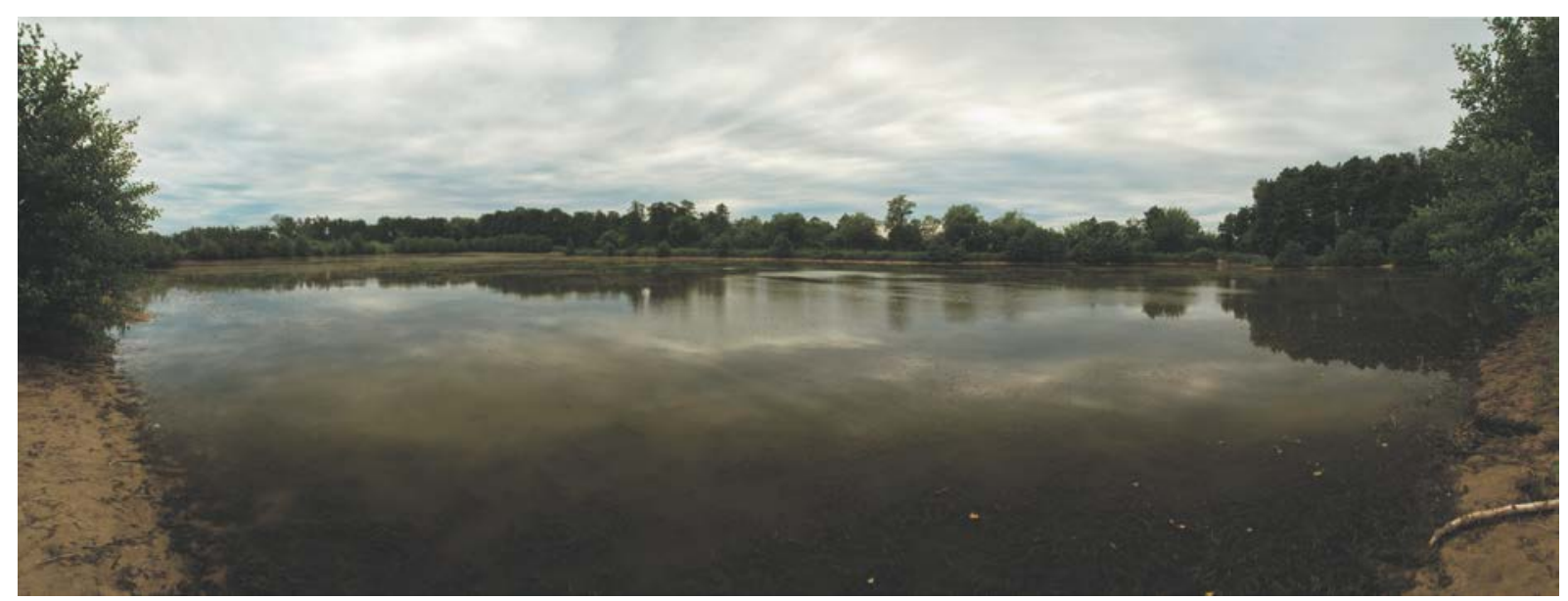

Fig 3: Marheček, wetlands (photo by Marek Svitok). 


\section{Results}

\section{PSYCHODIDAE \\ SYCORACINAE}

\section{Sycorax Haliday in Curtis, 1839}

\section{Sycorax silacea Haliday in Curtis, 1839}

Published records: Dobšinská l'adová jaskyňa cave (48) (Halgoš 1974, 1984); Baláže (6) (Ježek 1993); Hornád river basin (80), upper Váh river basin (345) (larvae, Bulánková 2003); Spády waterfall (279) (Ježek 2009a); Šišulákov mlyn mill (266) (Straka \& Majzlan 2013).

Material examined: Košická kotlina basin, Herl’any (73), 26.v.1979, SW, 1M, J. Ježek leg. \& det., slide Inv. No. 20609, NMPC, veg.: Fagus, Rosa, Rubus, Asperula, Urtica.

Comments: A locally common Europaen species.

\section{Sycorax slovaca Halgoš, 1975}

Published records: Soblahov (273) (Halgoš 1975, 1984); lower Váh river basin (149) (larvae, Bulánková 2003). Comments: Known only from Slovakia. In Jedlička \& Stloukalová (2001): vulnerable species, assessment of the current conservation status: CR.

\section{Sycorax tonnoiri Jung, 1954}

Published records: Vel'ký Sokol (359) (Halgoš 1975, 1984); Liptovský Hrádok (146), Tatranská Kotlina (317) (Ježek 1993); Hornád river basin (80), upper Váh river basin (345) (larvae, Bulánková 2003); Spády waterfall (279) (Ježek 2009a).

Material examined: Popradská kotlina basin, Skalnatý potok brook env. Tatranská Lomnica (267), 8.vii.1987, SW, 1M, J. Ježek leg. \& det., slide Inv. No. 19956, NMPC, veg.: Picea, Rubus, Petasites, Geum; Stolické vrchy Mts., Slanská dolina valley env. Rejdová (271), 17.vii.1989, SW, 1M, J. Ježek leg. \& det., slide Inv. No. 20661, NMPC, veg. Picea, Alnus, Rubus, Filipendula, Scirpus, Pteropsida.

Comments: A rare Europaen species. The assessment of the current conservation status: CR.

\section{TRICHOMYIINAE}

\section{Trichomyia Haliday in Curtis, 1839}

\section{Trichomyia urbica Haliday in Curtis, 1839}

Published records: Vráble (368) (Szabó 1965a,b, Halgoš 1984); Zbojnícka jaskyňa cave (387) (Košel \& Horváth 1996).

Comments: Not common Europaen species. In Jedlička \& Stloukalová (2001) vulnerable, the assessment of the current conservation status: CR.

\section{PSYCHODINAE \\ MORMIINI \\ MORMIINA}

Lepimormia Enderlein, 1937

\section{Lepimormia palposa (Tonnoir, 1919)}

Published record: Vysoké Tatry Mts. (379) (Enderlein 1937 - as tatrica, more see Ježek 1994).

Comments: A very rare Europaen species, the assessment of the current conservation status: CR. 


\section{Oomormia Ježek, 1984}

\section{Oomormia andrenipes (Strobl, 1910)}

Published records: Babí potok brook (3), Kardolina (108), Pod Muráňom (230), Rozpadliny (258), Suchá dolina valley (305), Suchý vrch hill (306), Úplaz (344), Zadná Kopa (381), Ždiar (389), (Ježek 1984b); Baláže (6), Črmné (42), Kl'ačianska dolina valley (112) (Ježek 1993).

Comments: A quite rare Europian species. The assessment of the current conservation status: CR.

\section{Promormia Ježek, 1983}

\section{Promormia eatoni (Tonnoir, 1940)}

Published records: Rimava river (255) (Ježek 2006a); Hrochotská dolina valley (87) (Ježek 2009a).

Material examined: Veporské vrchy Mts., Strieborný potok brook nr. Rimava (297), 16.x.1991, 1M, SW, P. Chvojka leg., J. Ježek det., slide Inv. No. 20400, NMPC.

Comments: A quite rare Europaen species. The assessment of the current conservation status: EN.

\section{Promormia silesiensis Ježek, 1983}

Published records: Rimava river (255) (Ježek 2006a); Hrochotská dolina valley (87) (Ježek 2009a).

Material examined: Veporské vrchy Mts., Strieborný potok brook nr. Rimava (297), 16.x.1991, 1M, SW, P. Chvojka leg., J. Ježek det., slide Inv. No. 20399, NMPC.

Comments: A rare Europaen species, the assessment of the current conservation status: CR.

\section{Taramormia Ježek, 1984}

\section{Taramormia tatrica Ježek, 1984}

Published records: Štrbské pleso mountain lake (294) (Szabó 1965b, Halgoš 1984); Košiar env. Ždiar (121), Zadná Kopa (381) (Ježek 1984b).

Comments: Probably rare central European species, known only from Slovakia and Czech Republic. The assessment of the current conservation status: CR.

\section{Yomormia Ježek, 1984}

\section{Yomormi furva (Tonnoir, 1940)}

Published record: Mokrý potok brook (184) (Ježek et al. 2012).

Comments: Not common Europaen species.

\section{PARAMORMIINI \\ PARAMORMIINA \\ Clogmia Enderlein, 1937}

\section{Clogmia albipunctata (Williston, 1893)}

Published records: Vrbany (371) (Ježek et al. 2012, Oboňa \& Ježek 2012a,b); Diviacka Nová Ves (46), Zvolen (396) (Oboňa \& Ježek 2012b).

Material examined: Trnavská pahorkatina hills, Piešt’any town (225), 29.viii.2012, 18.vii.-31.viii.2013, 1.30.ix.2013, 3M, HC, L. Balážiová leg., J. Ježek det., slides Inv. No. 20227, 20228 and 20229, NMPC; Trnavská pahorkatina hills, Trebatice (329), 30.viii.2012, HC, 1M, M. Baláž leg., J. Ježek det., slide Inv. No. 20246, NMPC; Zvolenská kotlina basin, Zvolen bus station (396), 297 m a.s.l., 15.viii.2013 and 23.ix.2013, 2M, HC, J. Oboňa leg., J. Ježek det., slides Inv. No. 20237 and 20238, NMPC; Hornonitrianska kotlina basin, Prievidza town (239), 3.ix.2013, 1F, HC, J. Oboňa leg., J. Ježek det., slide Inv. No. 20241, NMPC; Košická kotlina basin, Košice town (122), ix.2013, 1F, 1M, HC, K. Ox leg., J. Ježek det., slides Inv. No. 20240 and 20242, NMPC; Nitrianska pahorkatina hills, Rastislavice (248), 28.ix.2013, 7.x.2013, 1M, 1F, HC, P. Filipovič leg., J. Ježek det., slide Inv. No. 20244 and 20245, NMPC; Košická kotlina basin, Prešov bus station (238), 23.x.2013, 1F, 
HC, J. Oboňa leg., J. Ježek det., slide Inv. No. 20247, NMPC; Ondavská vrchovina hills, Mat'ovce (173), 27.x.2013, 1M, HC, M. Dobranský leg., J. Ježek det., slide Inv. No. 20239, NMPC.

Comments: Expansive, often synanthropic circumtropical and circumsubtropical species.

\section{Jungiella Vaillant, 1972 \\ Jungiella s. str.}

\section{Jungiella (Jungiella) hygrophila Ježek, 1987}

Material examined: Strážovské vrchy Mts., Diviacka Nová Ves env., well - Olavec (212 - Fig. 4, Map 4), 48 45' 10.8 " N, 18²9' 23.9" E, 290 m a.s.l., 22.vi.2012, SW, 1M, J. Oboňa leg., J. Ježek det., slide Inv. No. 20064, NMPC.

Distribution: Probably a central European species, uncommon, known only from the Czech Republic and Poland (Ježek 1987, Wagner 2011, Ježek \& Omelková 2012). New species for Slovakia.

\section{Jungiella (Jungiella) soleata (Walker, 1856)}

Published records: Liptovské Kl’ačany (144), Lubel’a (150) (Ježek 1993); Dudváh (58) (Straka 2009); Lukovský vrch hill (156) (Straka 2010); Šúr (309) (Straka \& Majzlan 2010a); Šišulákov mlyn mill (266) (Straka \& Majzlan 2013).

Comments: A common species distributed almost throughout Europe. Known from lowlands to mountains in shaded habitats with decaying organic matter.

\section{Jungiella (Jungiella) valachica (Vaillant, 1963)}

Materiala examined: Strážovské vrchy Mts., Diviacka Nová Ves env., well - Olavec (212- Fig 4, Map 4), 48 45' 10.8 " N, $18^{\circ}$ 29' 23.9" E, 290 m a.s.1., 22.vi.2012, SW, 1M, J. Oboňa leg., J. Ježek det., slide Inv. No. 20063, NMPC.

Distribution: A quite sporadic species, known from Poland, Czech Republic, Austria, Switzerland (Krek 1999, Wagner 2011, Ježek \& Omelková 2012), and previous records from Bosnia and Herzegovina, Croatia, Serbia (Kvifte et al. 2013). New species for Slovakia.

\section{Psychocha Ježek, 1983}

\section{Jungiella (Psychocha) acuminata (Szabó, 1960)}

Published records: Súl’ov (308) (larvae, Rozkošný 1971, Halgoš 1984); Šišulákov mlyn mill (266) (Straka \& Majzlan 2013).

Comments: A probably not common European species.

\section{Jungiella (Psychocha) laminata (Szabó, 1960)}

Materiala examined: Strážovské vrchy Mts., Diviacka Nová Ves env., well - Olavec (212 - Fig. 4, Map 4), $48^{\circ} 45^{\prime} 10.8$ " N, $18^{\circ} 29^{\prime}$ 23.9" E, 290 m a.s.l., 22.vi.2012, SW, 1M, J. Oboňa leg., J. Ježek det., slide Inv. No. 20031, NMPC.

Distribution: A very rare central European species, known only from the Czech Republic, Germany, Hungary and Serbia (Ježek 2003, Ježek \& Schacht 2006, Wagner 2011, Ježek \& Omelková 2012). New species for Slovakia. The assessment of the current conservation status: CR. 


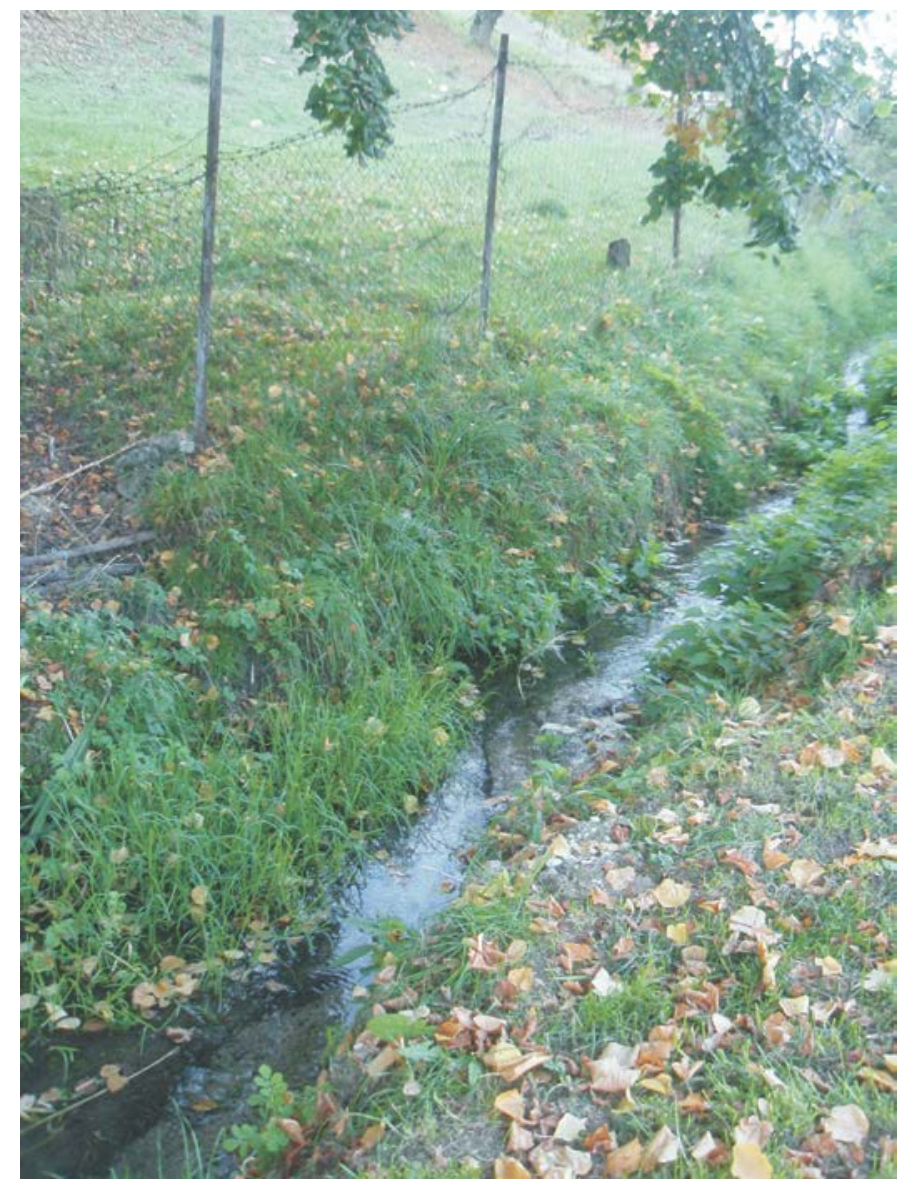

Fig 4: Olavec, well and small brook near village Diviacka Nová Ves (photo by Jozef Oboňa).

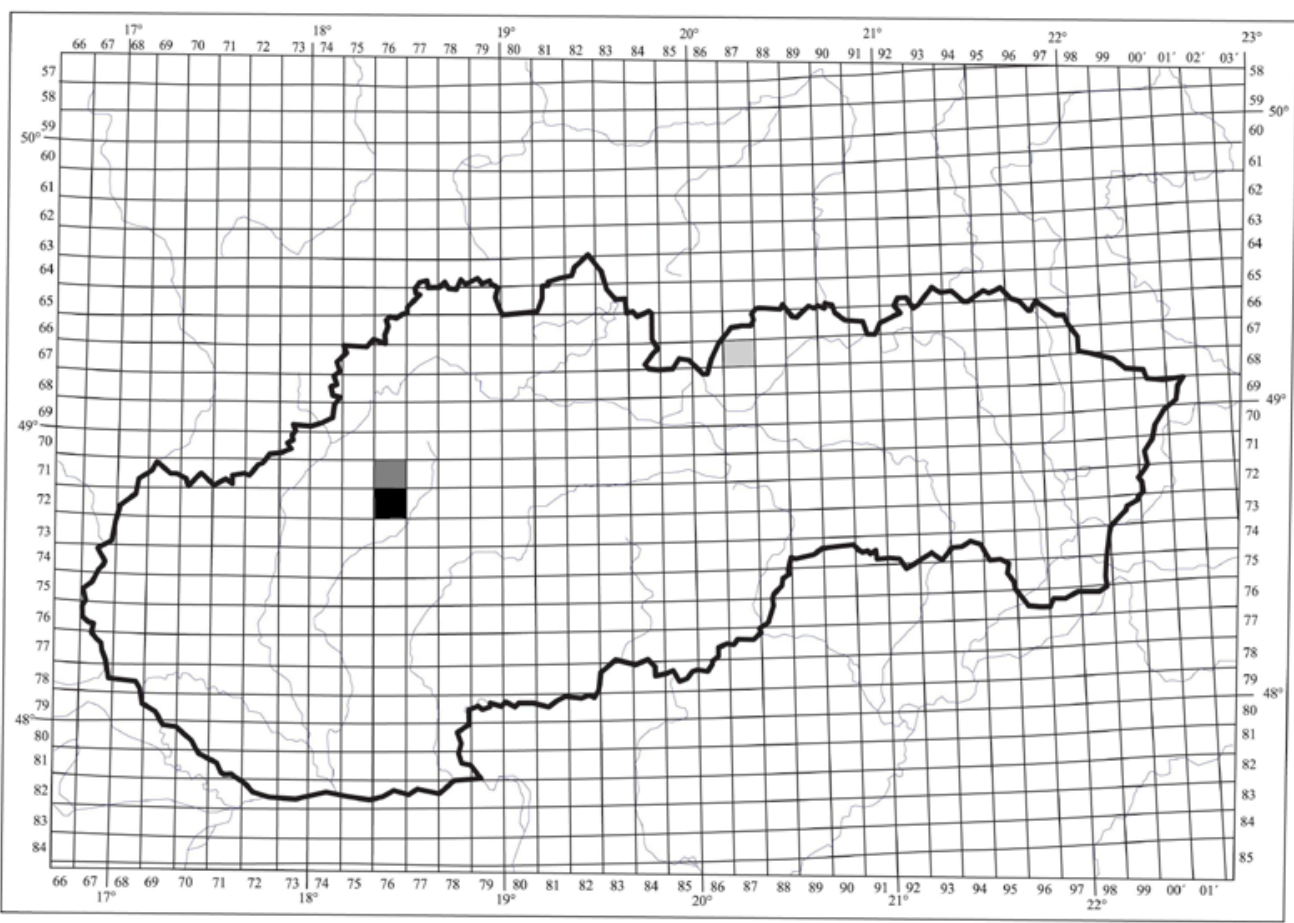

Map 4: Map showing Slovakian records of Jungiella (Jungiella) hygrophila Ježek, 1987, J. (J.) valachica (Vaillant, 1963) and J. (Psychocha) laminata (Szabó, 1960), all black square (DFS: 7276 - Olavec), Parajungiella prikryli Ježek, 1999 dark gray square (DFS: 7176 - Liešt’any) and Apsycha pusilla (Tonnoir, 1922) light gray square (DFS: 6787 - Ždiar). 


\section{Lepiseodina Enderlein, 1937}

\section{Lepiseodina tristis (Meigen, 1830)}

Published records: Diviacka Nová Ves (46), Vrbany (371) (Oboňa a Ježek 2012b).

Material examined: Strážovské vrchy Mts., Diviacka Nová Ves (46), ii.-v.2013, all reared from Quercus and Fagus, 20.ii.2013, 1M, reared from Ulmus sp., J. Oboňa leg., J. Ježek det., slides Inv. No. 20067, 20076, 20077, 20079, 20080, 20288, 20243, NMPC.

Comments: A rare dendrolimnobiontic species. The assessment of the current conservation status: CR.

\section{Panimerus Eaton, 1913}

\section{Panimerus notabilis (Eaton, 1893)}

Published records: Oravský Podzámok (219) (Szabó 1965a,b, Halgoš 1984); Javorina (101), Špania Dolina (280) (Ježek 1993); upper Váh river basin (345) (larvae, Bulánková 2003).

Material examined: Východoslovenská pahorkatina hills, Vranov nad Topl'ou (369), 25.v.1979, SW, 1M, J. Ježek leg. \& det., slide Inv. No. 20259, NMPC, veg.: Salix, Juglans, Alnus, Cerasus, Urtica, Chelidonium; Východoslovenská rovina flat, Král’ovský Chlmec (126), 29.v.1979, SW, 1M, J. Ježek leg. \& det., slide Inv. No. 20341, NMPC, veg.: Salix, Populus, Alisma, Ranunculus, Carex; Východoslovenská pahorkatina hills, Michal'any (180), 23.viii.1983, SW, 1M, J. Ježek leg. \& det., slide Inv. No. 20230, NMPC, veg.: Salix, Populus, Urtica, Heracleum, Plantago, Ranunculus, Lychnis; Laborecká vrchovina hills, Krásny Brod env. Medzilaborce, brook, well (128), 10.ix.1991, SW, 1M, J. Ježek leg. \& det., slide Inv. No. 20116, NMPC, veg.: Alnus, Salix, Corylus, Rubus, Caltha, Mentha, Carex, Dipsacus, Musci.

Comments: A common species known from Europe and Iran.

\section{Parajungiella Vaillant, 1972}

\section{Parajungiella consors (Eaton, 1893)}

Published record: Nižná Myšl’a (199) (Ježek 1984a).

Comments: A rare Europaen species, distributed from Great Britain to Denmark, Belgium, the Netherlands, Czech Republic and Slovakia.

\section{Parajungiella ellisi (Withers, 1987)}

Published record: Tatranské Matliare (321) (Ježek 2003).

Comments: A rare Europaen and West-Siberian species, the assessment of the current conservation status: CR.

\section{Parajungiella longicornis (Tonnoir, 1919)}

Published records: Bystrá (29), Dúbrava (56), Javorina (101), Liptovské Kl'ačany (144), Lubel'a (150), Pavčina Lehota (223), Tatranská Lomnica (319) (Ježek 1993); Hrochotská dolina valley (87) (Ježek 2009a).

Material examined: Košická kotlina basin, Dulova Ves nr. Prešov (59), 23.v.1979, SW, 1M, J. Ježek leg. \& det., slide Inv. No. 19979, NMPC, veg.: Quercus, Scirpus, Urtica, Ranunculus, Rosa; Východoslovenská pahorkatina hills, Čaklov (30), 25.v.1979, SW, 1M, J. Ježek leg. \& det., slide Inv. No. 20403, NMPC, veg.: Populus, Juglans, Sambucus, Crataegus, Urtica, Lappa; Východoslovenská rovina flat, Sečovce env.Trebišov (264), 27.v.1979, SW, 1M, J. Ježek leg. \& det., slide Inv. No. 19959, NMPC, veg.: Alnus, Salix, Lappa, Symphytum, Ranunculus; Východoslovenská pahorkatina hills, Michal'any (180), 23.viii.1983, SW, 1 M, J. Ježek leg. \& det., slide Inv. No. 20234, NMPC, veg.: Salix, Populus, Urtica, Heracleum, Plantago, Ranunculus, Lychnis; Liptovská kotlina basin, Pavčina Lehota nr. Liptovský Mikuláš (223), 10.vi.1987, SW, 1M, J. Ježek leg. \& det., slide Inv. No. 19943, NMPC, veg.: Salix, Rubus, Caltha, Alisma, Filipendula, Geum, Urtica; Popradská kotlina basin, Lučivná - Sviniarka (153), 938 m a.s.l., 13.vii.1987, SW, 1M, J. Ježek leg. \& det., slide Inv. No. 19940, NMPC, veg.: Picea, Alnus, Caltha, Filipendula, Equisetum; Podunajská rovina flat, Šúr (309), 7.viii.2009, MT, 1M, J. Lukáš leg., J. Ježek det., slide Inv. No. 20638, NMPC; Hornonitrianska kotlina basin, Liešt'any, meanders of a brook (140), 23.v.2013, SW, 1 M, J. Oboňa leg., J. Ježek det., slide Inv. No. 20074, NMPC.

Comments: Generally a very common European and Westsiberian species. 


\section{Parajungiella prikryli Ježek, 1999}

Materiala examined: Hornonitrianska kotlina basin, Liešt'any, meanders of a brook (140 - Map 4), 48 50' 41.3" N 18²8' 14.4" E, 290 m a.s.l., 23.v.2013, SW, 1M, J. Oboňa leg., J. Ježek det., slide Inv. No. 20073, NMPC.

Distribution: Probably a rare central European species, known only from the Czech Republic - Ježek (1999), Ježek \& Omelková (2012). New species for Slovakia. The assessment of the current conservation status: VU.

\section{Parajungiella pseudolongicornis (Wagner, 1975)}

Published records: Sečovce (264) (Ježek 2003); Hrochotská dolina valley (87) (Ježek 2009a).

Material examined: Východoslovenská rovina plain, Kapušianske Kl’ačany (107), 29.v.1979, SW, 1M, J. Ježek leg., det., slide Inv. No. 20588, NMPC, veg.: Quercus, Fraxinus, Populus, Salix, Iris; Liptovská kotlina basin, Pavčina Lehota nr. Liptovský Mikuláš (223), 10.vi.1987, SW, 1M, J. Ježek leg. \& det., slide Inv. No. 19951, NMPC, veg.: Salix, Rubus, Caltha, Alisma, Filipendula, Geum, Urtica.

Comments: A rare European species, the assessment of the current conservation status: CR.

\section{Parajungiella serbica (Krek, 1985)}

Published record: Lubel'a (149) (Ježek 2003).

Material examined: Východoslovenská pahorkatina hills, Michal'any (180), 23.viii.1983, SW, 1M, J. Ježek leg. \& det., slide Inv. No. 20233, NMPC, veg.: Salix, Populus, Urtica, Heracleum, Plantago, Ranunculus, Lychnis.

Comments: Quite a rare species, known from Czech Republic, Slovakia, Greece and Serbia. The assessment of the current conservation status: CR.

\section{Paramormia Enderlein, 1935 Duckhousiella Vaillant, 1972}

\section{Paramormia (Duckhousiella) ustulata (Walker, 1856)}

Published record: Nová Vieska (209) (Ježek 1990a).

Material examined: Laborecká vrchovina hills, Dedačov (44), 9.ix.1979, SW, 1M, J. Ježek leg. \& det., slide Inv. No. 20595, NMPC, veg.: Salix, Alnus, Corylus, Asarum, Urtica; Hornonitrianska kotlina basin, Koš wetlands (119), 26.ix.2012, SW, 1M, J. Oboňa leg., J. Ježek det., slide Inv. No. 20020, NMPC; Hornonitrianska kotlina basin, Prievidza (239), 4.x.2012, HC, 1F, J. Oboňa leg., J. Ježek det., slide Inv. No. 20021, NMPC; Borská nížina lowlands, Marheček wetlands (170), 19.iv.2013, SW, 1 M, J. Oboňa leg., J. Ježek det., slide Inv. No. 20072, NMPC; Tríbeč hill, Hrušov (91), 24.iv.2013, SW, 1M, J. Oboňa leg., J. Ježek det., slide Inv. No. 20055, NMPC.

Comments: A species known from Europe and north Africa.

\section{Paramormia s. str.}

\section{Paramormia (Paramormia) polyascoidea (Krek, 1971)}

Published records: Račková dolina valley (244) (Rozkošný 1971, Halgoš 1984); Dúbrava (56), Gerlachov (66), Lubel’a (150), Mlynica (183), Štôla (292), Tatranská Lesná (318) (Ježek 1993).

Material examined: Košická kotlina basin, Dulova Ves nr. Prešov (59), 23.v.1979, SW, 1M, J. Ježek leg. \& det., slide Inv. No. 19980, NMPC, veg.: Quercus, Scirpus, Urtica, Ranunculus, Rosa; Košická kotlina basin, Prešov (238), 23.v.1979, SW, 1M, J. Ježek leg. \& det., slide Inv. No. 20009, NMPC; Východoslovenská pahorkatina hills, Čaklov (30), 25.v.1979, SW, 1M, J. Ježek leg. \& det., slide Inv. No. 20402, NMPC, veg.: Populus, Juglans, Sambucus, Crataegus, Urtica, Lappa; Východoslovenská pahorkatina hills, Vranov nad Topl'ou (369), 25.v.1979, SW, 1M, J. Ježek leg. \& det., slide Inv. No. 20260, NMPC, veg.: Salix, Juglans, Alnus, Cerasus, Urtica, Chelidonium; Východoslovenská pahorkatina hills, Michal'any (180), 23.viii.1983, SW, 1M, J. Ježek leg. \& det., slide Inv. No. 20231, NMPC, veg.: Salix, Populus, Urtica, Heracleum, Plantago, Ranunculus, Lychnis; Popradská kotlina basin, Nová Lesná brook (206), 23.viii.1983, SW, 1M, J. Ježek leg. \& det., slide Inv. No. 20102, NMPC, veg.: Alnus, Salix, Urtica; Liptovská kotlina basin, Pavčina Lehota nr. Liptovský Mikuláš (223), 10.vi.1987, SW, 1M, J. Ježek leg. \& det., slide Inv. No. 19953, NMPC, veg.: Salix, Rubus, Caltha, Alisma, Filipendula, Geum, Urtica; Popradská kotlina basin, Lučivná - Sviniarka env., well, brook (153), 938 m a.s.l., 13.vii.1987, SW, 1 M, J. Ježek leg. \& det., slide Inv. No. 19984, NMPC, veg.: Picea, Alnus, Caltha, Filipendula, Equisetum. 
Comments: A common European and Westsiberian species.

\section{Phyllotelmatoscopus Vaillant, 1982}

\section{Paramormia (Phyllotelmatoscopus) acuta (Krek, 1971)}

Published record: Furmanec river (63) (Ježek 2006a).

Comments: A rare European species. The assessment of the current conservation status: NS.

\section{Peripsychoda Enderlein, 1935}

\section{Peripsychoda auriculata (Haliday in Curtis, 1839)}

Published records: Bardejov (11), Oravský Podzámok (219) (Szabó 1965a,b); Soblahov (273) (Halgoš 1973); Bardejov (11), Oravský Podzámok (219), Rimavská Sobota (256), Soblahov (273) (Halgoš 1984); Hrochotská dolina valley (87) (Ježek 2009a).

Material examined: Východoslovenská pahorkatina hills, Slančík (270), 28.v.1979, SW, 1M, J. Ježek leg. \& det., slide Inv. No. 20090, NMPC, veg.: Salix, Alnus, Corylus, Urtica, Asarum; Východoslovenská rovina flat, Somotor (277), 30.v.1979, SW, 1M, J. Ježek leg. \& det., slide Inv. No. 20144, NMPC. veg.: Salix, Nymphaea, Lemna, Urtica; Popradská kotlina basin, Lučivná - Sviniarka env., well, brook (153), 938 m a.s.l., 13.vii.1987, SW, 1M, J. Ježek leg. \& det., slide Inv. No. 19982, NMPC. veg.: Picea, Alnus, Caltha, Filipendula, Equisetum; Popradská kotlina basin, Červený potok brook - Nová Lesná (34), 22.vii.1990, SW, 1M, P. Chvojka leg., J. Ježek det., slide Inv. No. 20411, NMPC; Bukovské vrchy Mts., Stinská slatinka meadow (291), 700 m a.s.l., 20.vii.2005, SW, 1M, P. Kment leg., J. Ježek det., slide Inv. No. 20435 NMPC; Malé Karpaty Mts., Fialkový potok brook (62), 247 m a.s.l., 21.v.2011, SW, 1M, J. Lukáš leg., J. Ježek det., slide Inv. No. 20631, NMPC; Malé Karpaty Mts., Mokrý potok brook (184), 309 m a.s.l., 25.viii.2011, SW, 1M, J. Lukáš leg., J. Ježek det., slide Inv. No. 20629, NMPC.

Comments: A very common European and Transcaucasian species.

\section{Peripsychoda fusca (Macquart, 1826)}

Published records: Fialkový potok brook (62), Vel'kolúcky potok brook (355) (Ježek et al. 2012).

Comments: Only locally common European species. The assessment of the current conservation status: CR.

\section{Psycmera Ježek, 1984}

\section{Psycmera integella (Jung, 1956)}

Published records: Brezno (22), Liptovské Kl’ačany (144), Lubel’a (150), Pavčina Lehota (223), Ploštín (229) (Ježek 1993); Nad Šenkárkou (193) (Straka \& Majzlan 2006).

Material examined: Liptovská kotlina basin, Pavčina Lehota nr. Liptovský Mikuláš (223), 10.vi.1987, SW, 1M, J. Ježek leg. \& det., slide Inv. No. 19947, NMPC, veg.: Salix, Rubus, Caltha, Alisma, Filipendula, Geum, Urtica.

Comments: A European and West-Siberian species, known mainly from swampy alder-woods. The assessment of the current conservation status: $\mathrm{CR}$.

\section{Sciria Enderlein, 1935}

\section{Sciria advena (Eaton, 1893)}

Published records: Smrekovec (272) (Straka \& Majzlan 2009); Diviacka Nová Ves (46), Vrbany (370) (Oboňa \& Ježek 2012b).

Comments: A rare European species associated with dendrothelmata. The assessment of the current conservation status: CR. 


\section{Telmatoscopus Eaton, 1904}

\section{Telmatoscopus carpathicus Ježek, 1988}

Published records: Haliar (69), Pod Príslopom (231) (Ježek 1988); Čierna Lehota (37) (Straka \& Majzlan 2007).

Material examined: Liptovská kotlina basin, Pavčina Lehota nr. Liptovský Mikuláš (223), 10.vi.1987, SW, 1M, J. Ježek leg. \& det., slide Inv. No. 19949, NMPC, veg.: Salix, Rubus, Caltha, Alisma, Filipendula, Geum, Urtica.

Comments: An extremely rare species, known only from Slovakia and the Czech Republic. The assessment of the current conservation status: VU.

\section{Telmatoscopus carthusianus (Vaillant, 1972)}

Published record: Hrochotská dolina valley (87) (Ježek 2009a).

Material examined: Strážovské vrchy Mts., Diviacka Nová Ves (46), 22.v.2012, HC, 1M, J. Oboňa leg., J. Ježek det., slide Inv. No. 20026, NMPC.

Comments: Lokally a relative rare European species, sometimes collected together with T. gressicus (Vaillant, 1972).

\section{TRICHOPSYCHODINA \\ Philosepedon Eaton, 1904}

\section{Philosepedon s. str.}

\section{Philosepedon (Philosepedon) austriacum Vaillant, 1974}

Published records: Hiadel'ské sedlo saddleback (75), Ploštín (229) (Ježek 1993).

Material examined: Kremnické vrchy Mts., Železná Breznica env., brook (390), 29.iv.2012, SW, 1M, J. Oboňa leg., J. Ježek det., slide Inv. No. 20048, NMPC.

Comments: Lokally a relative rare European species.

\section{Philosepedon (Philosepedon) humerale (Meigen, 1818)}

Published records: Zbojská (385) (Szabó 1965a,b, Halgoš 1984); Liptovský Hrádok (146) (Ježek 1993); Zbojnícka jaskyňa cave (388) (Košel \& Horváth 1996).

Material examined: Kremnické vrchy Mts., Železná Breznica env., brook (390), 30.iv.2013, SW, 1M, J. Oboňa leg., J. Ježek det., slide Inv. No. 20061, NMPC.

Comments: Generally a very common and widely distributed European and North African species.

\section{Trichosepedon Krek, 1999}

\section{Philosepedon (Trichosepedon) balkanicum Krek, 1971}

Published records: Babí potok brook (3), Dolina 7 prameňov valley (49), Košiar env. Javorina (120), Patočiny (222) (Ježek 1993); Košiar env. Ždiar (121) (Ježek 1995).

Material examined: Popradská kotlina basin, Tatranská Polianka (320), 21.viii.1983, SW, 1M, J. Ježek leg. \& det., slide Inv. No. 20428, NMPC, veg.: Picea, Alnus, Rubus, Caltha, Lysimachia, Filipendula, Urtica; Slovenský raj, Vlčia jaskyňa cave (363), 4.viii.1994, HC, 1M, V. Košel leg., J. Ježek det., slide Inv. No. 7824, NMPC; Slovenský raj, Vojenská jaskyňa cave (365), 5.viii.1994, HC, 1M, V. Košel leg., J. Ježek det., slide Inv. No. 19963, NMPC.

Comments: Generally a rare European species. The assessment of the current conservation status: CR.

\section{Threticus Eaton, 1904}

\section{Threticus balkaneoalpinus Krek, 1972}

Published records: Kardolina (108), Pod Príslopom (231), Podspády (234), Tatranská Kotlina (317), Tokárensky potok brook (326), Vojtasova pol'ana meadow (366) (Ježek 1993); Hrochotská dolina valley (87), Zadná Pol’ana (382) (Ježek 2009a). 
Material examined: Laborecká vrchovina hills, Krásny Brod env. Medzilaborce, brook, well (128), 10.ix.1991, SW, 1M, J. Ježek leg. \& det., slide Inv. No. 20111, NMPC, veg.: Alnus, Salix, Corylus, Rubus, Caltha, Mentha, Carex, Dipsacus, Musci.

Comments: A not common Europaen species.

\section{Threticus incurvus Krek, 1972}

Published records: Baláže (6), Čierna dolina valley (36), Dolný Jelenec (52), Il'anovská dolina valley (95), Špania Dolina (280), Tatranská Kotlina (317), Ul'anka (341) (Ježek 1993); Tatranská Kotlina (317) (Ježek 1995). Material examined: Čierna hora Mt., Čermel’ nr. Košice (31), 19.v.1980, SW, 1M, J. Ježek leg. \& det., slide Inv. No. 20365, NMPC, veg.: Alnus, Salix, Carpinus, Petasites, Urtica.

Comments: A rare Europaen species. The assessment of the current conservation status: EN.

\section{Threticus lucifugus (Walker, 1856)}

Published records: Zbojská (388) (Szabó 1960, 1965a,b, Halgoš 1984); Marková dolina valley (171), Piesky (224), Prostredná stream (241), Staré Hory (287), Ul’anka (341), Východná (372) (Ježek 1993); Hrončecký grúň slope (90) (Ježek 2009a); Hron river basin (89), upper Váh river basin (345) (larvae, Bulánková 2003).

Material examined: Popradská kotlina basin, Tatranská Polianka (320), 21.viii.1983, SW, 1M, J. Ježek leg. \& det., slide Inv. No. 20426, NMPC, veg.: Picea, Alnus, Rubus, Caltha, Lysimachia, Filipendula, Urtica; Nízke Tatry Mts., Liptovská Lúžna, brook (141), 13.ix.1985, SW, 1M, J. Ježek leg. \& det., slide Inv. No. 20321, NMPC, veg.: Picea, Sphagnum, Caltha, Juncus, Mentha, Poaceae; Laborecká vrchovina hills, Korejovce (117), 13.ix.1991, SW, 1M, J. Ježek leg. \& det., slide Inv. No. 20163, NMPC, veg.: Salix, Alnus, Petasites, Dipsacus, Impatiens, Musci, Poaceae; Spišsko-šarišské medzihorie Imts., Stará Lubbovňa (284), 28.ix.1992, SW, 1M, J. Ježek leg. \& det., slide Inv. No. 20655, NMPC, Alnus, Salix, Prunus, Corylus, Ribes, Colchicum, Geranium, Mentha, Urtica.

Comments: A Europaen species.

\section{Threticus negrobovi Vaillant, 1972}

Published records: Dolina 7 prameňov valley (49), Kežmarské Žl'aby (111), Tatranská Kotlina (317), Ždiar (389) (Ježek 1993); Hrochotská dolina valley (87) (Ježek 2009a).

Comments: A rare Europaen species. The assessment of current conservation status: CR.

\section{Threticus silvaticus Ježek, 1985}

Published records: Hiadel'ské sedlo saddleback (75), Marková dolina valley (171), Piesky (224), Prostredná stream (241), Východná (372) (Ježek 1993).

Material examined: Ondavská vrchovina hills, Nová Polianka env. Svidník (207), 12.xi.1991, SW, 1M, J. Ježek leg. \& det., slide Inv. No. 20383, NMPC, veg.: Salix, Alnus, Corylus, Rubus, Typha, Lappa, Urtica, Daucaceae; Čergov Mts., Olejníkov env. Majdan (213), 26.ix.1992, SW, 1M, J. Ježek leg. \& det., slide Inv. No. 20499, NMPC, veg.: Alnus, Salix, Corylus, Urtica; Spišsko-šarišské medzihorie Imts., Čírč (41), 27.ix.1992, SW, 1M, J. Ježek leg. \& det., slide Inv. No. 20401, NMPC, veg.: Fagus, Pinus, Caltha, Mentha, Musci, Pteropsida.

Comments: Not common Europaen species, the assessment of the current conservation status: VU.

\section{Trichopsychoda Tonnoir, 1922}

\section{Trichopsychoda hirtella (Tonnoir, 1919)}

Published records: Tále (316) (Ježek 1993); Hrochotská dolina valley (87) (Ježek 2009a).

Material examined: Malé Karpaty Mts., Fialkový potok brook (62), 247 m a.s.l., 4.ix.2011, SW, 1M, J. Lukáš leg., J. Ježek det., slide Inv. No. 20635, NMPC; Hornonitrianska kotlina basin, Vrbany env. Diviacka Nová Ves (371), 20.v.2012, SW, 1F, J. Oboňa leg., J. Ježek det., slide Inv. No. 20053, NMPC.

Comments: Generally a common Europaen species. 


\section{PSYCHODINI}

Apsycha Ježek, 2007

\section{Apsycha pusilla (Tonnoir, 1922)}

Material examined: Podtatranská brázda furrow, Ždiar (389 - Map 4), 26.ix.-2.x.1993, YPT, 1M, P. Chvojka leg., J. Ježek det., slide Inv. No. 20483, NMPC.

Distribution: Species mainly of cow-sheds, therefore overlooked, previously recorded only from USA, Czech Republic and Poland (Ježek 2007). New species for Slovakia.

\section{Chodopsycha Ježek, 1984}

\section{Chodopsycha buxtoni (Withers, 1988)}

Published record: Nová Sedlica (208) (Ševčík 2004, 2010).

Material examined: Bukovské vrchy Mts., Topol’a (328), 8.ix.1979, SW, 1M, J. Ježek leg. \& det., slide Inv. No. 20418, NMPC, veg.: Alnus, Corylus, Urtica.

Comments: Probably rare European species, associated with fungi. The assessment of the current conservation status: NS.

\section{Chodopsycha lobata (Tonnoir, 1940)}

Published records: Brtkovica (23), Bukovecká dolina valley (28), Bystrá (29), Črmné (42), Hiadel'ská dolina valley (74), Horné Lazy (81), Kochul'a (114), Podbanské (232), Tri studničky well (332), Vyšné Hágy (376) (Ježek 1993); Nová Sedlica (208) (Ševčík 1994); Kyslinky (136), Zadná Pol’ana (382) (Ježek 2009a); Hrochotská dolina valley (87), Nová Sedlica (208), Muránska planina plain (191) (Ševčík 2010).

Material examined: Bukovské vrchy Mts., Kolbasov (116), 6.ix.1979, SW, 1F, J. Ježek leg. \& det., slide Inv. No. 20610, NMPC, veg.: Sambucus, Salix, Alnus, Urtica; Vihorlatské vrchy Mts., Zemplínske Hámre, brook (392), 6.ix.1979, SW, 1M, J. Ježek leg. \& det., slide Inv. No. 20338, NMPC, veg.: Salix, Alnus, Urtica; Vysoké Tatry Mts., Grúnik (68), 15.viii.1983, SW, 1M, J. Ježek leg. \& det., slide Inv. No. 20501, NMPC, veg.: Picea; Popradská kotlina basin, Eurocamp FICC env. Tatranská Lomnica (61), 24.viii.1983, SW, 1M, J. Ježek leg. \& det., slide Inv. No. 20591, NMPC, veg.: Picea, Pinus, Alnus, Frangula, Sorbus, Caltha, Urtica; Nízke Tatry Mts., Bansko, brook (nr. Liptovská Lúžna) (9), 1181 m a.s.l., 13.ix.1985, SW, 1F, J. Ježek leg. \& det., slide Inv. No. 20562, NMPC, veg.: Picea, Betula, Sambucus, Rubus, Mentha; Nízke Tatry Mts., Liptovská Lúžna (141), 13.ix.1985, SW, 1F, J. Ježek leg. \& det., slide Inv. No. 20473, NMPC, veg.: Picea, Mentha, Urtica; Zvolenská kotlina basin, Vážna nr. Lučatín (350), 15.ix.1985, SW, 1F, J. Ježek leg. \& det., slide Inv. No. 20329, NMPC, veg.: Picea, Alnus, Petasites, Urtica; Nízke Tatry Mts., Púchalky (243), 9.ix.1986, SW, 1F, J. Ježek leg. \& det., slide Inv. No. 20297, NMPC, veg.: Picea, Rubus, Pteropsida; Stolické vrchy Mts., Muránska Zdychava (192), 19.vii.1989, SW, 1M, J. Ježek leg. \& det., slide Inv. No. 20140, NMPC, veg.: Alnus, Rubus, Filipendula, Impatiens, Caltha, Peropsida; Stolické vrchy Mts., Muránska Lehota (190), 20.vii.1989, SW, 1M, J. Ježek leg. \& det., slide Inv. No. 19962, NMPC, veg.: Alnus, Corylus, Petasites, Scirpus, Impatiens, Urtica; Laborecká vrchovina hills, Malá Pol’ana (164), 10.ix.1991, SW, 1M, J. Ježek leg. \& det., slide Inv. No. 20352, NMPC, veg.: Fagus, Carpinus, Corylus, Rubus, Melampyrum, Daucaceae; Ondavská vrchovina hills, Krajné Čierno (125), 12.ix.1991, SW, 1F, J. Ježek leg. \& det., slide Inv. No. 20440, NMPC, veg.: Fagus, Carpinus, Betula, Salix, Petasites, Juncus, Impatiens, Poaceae; Ondavská vrchovina hills, Bardejovské Kúpele (12), 15.ix.1991, SW, 1F, J. Ježek leg. \& det., slide Inv. No. 20465, NMPC, veg.: Tilia, Salix, Corylus, Frangula, Rubus, Daucaceae, Lamiaceae; Spišská Magura Mts., Osturňa (220), 6.x.1991, SW, 1F, P. Chvojka leg., J. Ježek det., slide Inv. No. 20652, NMPC; Spišsko-šarišské medzihorie Imts., Stará Lubovňa (284), 28.ix.1992, SW, 1M, J. Ježek leg. \& det., slide Inv. No. 20656, NMPC, veg.: Alnus, Salix, Prunus, Corylus, Ribes, Colchicum, Geranium, Mentha, Urtica; Bukovské vrchy Mts., Jarabá skala rock (99), 12.viii.2003, SW, 1M, J. Ševčík leg., J. Ježek det., slide Inv. No. 12021, NMPC; Vysoké Tatry Mts., Úplaz env. Javorová dolina valley (344), 27.viii.2004, reared from fungus, 1M, V. Košel leg., J. Ježek det., slide Inv. No. 13050, NMPC.

Comments: A very common European - Transcaucasian species, widespread in lowlands as well as in hills and mountains; associated with fungi (Ježek \& Omelkévá 2012, Ježek et al. 2013). 


\section{Copropsychoda Vaillant, 1971}

\section{Copropsychoda brevicornis (Tonnoir, 1940)}

Published record: Brtkovica (23) (Ježek 1993).

Material examined: Bukovské vrchy Mts., Zboj (386), 7.ix.1979, SW, 1F, J. Ježek leg. \& det., slide Inv. No. 20532, NMPC, veg.: Alnus, Corylus, Humulus, Mentha; Nízke Tatry Mts., Vel'ký Brankov (356), 12.ix.1985, SW, 1F, J. Ježek leg. \& det., slide Inv. No. 20489, NMPC, veg.: Picea, Rubus, Musci; Čergov Mts., Hromacký vrch hill env. Livov (88), 830 m a.s.l., 26.ix.1992, SW, 1M, J. Ježek leg. \& det., slide Inv. No. 20582, NMPC, veg.: Fagus, Picea, Pinus, Abies, Betula, Mentha, Poaceae.

Comments: A saprophagous species with a western Palaearctic distribution. Probably common in Slovakia, however, overlooked so far.

\section{Logima Eaton, 1904}

\section{Logima albipennis (Zetterstedt, 1850)}

Published records: Bardejov (11) (as Psychoda severini Tonnoir, 1922), Demänovská dolina valley (45), Ružomberok (261) (Szabó 1965a,b); Bratislava (19), Malacky (167) (as Psychoda (Psychoda) severini partenogenetika Tonnoir, 1922) (Halgoš 1973); Nižná Myšla (199) (Ježek 1983); Bratislava (19), Demänovská dolina valley (45), Malacky (167), Ružomberok (as Psychoda severini Tonnoir, 1920) (261), Bardejov (11) (Halgoš 1984); Brtkovica (23), Dúbrava (56), Gerlachov (66), Hiadel'ská dolina valley (74), Kl'ačianská dolina valley (112), Ludrová (154), Lupčianska dolina valley (157), Mengusovce (178), Mlynica (183), Piesky (224), Ploštín (229), Prostredná stream (241), Ružomberok (261), Tichá dolina valley (323), Tri studničky well (332), Východná (372), Vyšné Hágy (376) (Ježek 1993); Hrochotská dolina valley (87) (Ježek 2009a).

Material examined: Košická kotlina basin, Košický Klečenov (124), 26.v.1979, SW, 1F, J. Ježek leg. \& det., slide Inv. No. 20524, NMPC, veg.: Fagus, Asarum, Trifolium, Asperula; Východoslovenská rovina plain, Vel'ký Horeš (358), 29.v.1979, SW, 1F, J. Ježek leg. \& det., slide Inv. No. 20540, NMPC, veg.: Salix, Juglans, Iris, Carex, Dactylis, Alisma; Beskydské predhorie Mts., Kamienka (106), 5.ix.1979, SW, 1F, J. Ježek leg. \& det., slide Inv. No. 20570, NMPC, veg.: Fagus, Betula, Picea, Petasites; Laborecká vrchovina hills, Belá nad Cirochou brook (14), 6.ix.1979, SW, 1F, J. Ježek leg. \& det., slide Inv. No. 20000, NMPC, veg.: Salix, Alnus, Caltha, Urtica; Laborecká vrchovina hills, Dedačov (44), 9.ix.1979, SW, 1M, J. Ježek leg. \& det., slide Inv. No. 20600, NMPC, veg.: Salix, Alnus, Corylus, Asarum, Urtica; Laborecká vrchovina hills, Hankovce (70), 9.ix.1979, SW, 1M, J. Ježek leg. \& det., slide Inv. No. 20515, NMPC, veg.: Salix, Sambucus, Cornus, Urtica; Volovské vrchy Mts., Rudňany (259), 21.v.1980, SW, 1F, J. Ježek leg. \& det., slide Inv. No. 20535, NMPC, veg.: Picea, Caltha, Mentha; Vysoké Tatry Mts., Škaredý žl'ab trough env. Kôprová dolina valley (268), 15.viii.1983, SW, 1M, J. Ježek leg. \& det., slide Inv. No. 20518, NMPC, veg.: Picea; Vysoké Tatry Mts., Pálenica (221), 15.viii.1985, SW, 1F, J. Ježek leg. \& det., slide Inv. No. 20527, NMPC, veg.: Picea, Sorbus, Acer, Petasites; Nízke Tatry Mts., Vel'ký Brankov (356), 12.ix.1985, SW, 1F, J. Ježek leg. \& det., slide Inv. No. 20492, NMPC, veg.: Picea, Rubus, Musci; Nízke Tatry Mts., Bansko brook (nr. Liptovská Lúžna) (9), 1181 m a.s.l., 13.ix.1985, SW, 1F, J. Ježek leg. \& det., slide Inv. No. 20566, NMPC, veg.: Picea, Betula, Sambucus, Rubus, Mentha; Nízke Tatry Mts., Brusno env. Brezno (24), 16.ix.1985, SW, 1F, J. Ježek leg. \& det., slide Inv. No. 20101, NMPC, veg.: Salix, Alnus, Corylus, Urtica, Asarum; Stolické vrchy Mts., Slanská dolina valley env. Rejdová (271), 18.vii.1989, SW, 2F, J. Ježek leg. \& det., slides Inv. No. 20607, 20662, NMPC, veg.: Picea, Caltha, Mentha, Impatiens, Pteropsida; Revúcka vrchovina Mts., Revúca (253), 19.vii.1989, SW, 1F, J. Ježek leg. \& det., slide Inv. No. 20601, NMPC, veg.: Salix, Populus, Rubus, Impatiens, Urtica, Humulus, Daucaceae, Poaceae; Ondavská vrchovina hills, Rakovč́́k (246), 12.ix.1991, SW, 1F, J. Ježek leg. \& det., slide Inv. No. 20295, NMPC, veg.: Alnus, Prunus, Corylus, Sambucus, Salix, Cirsium, Poaceae, Daucaceae; Ondavská vrchovina hills, Jurkova Vol’a (103), 14.ix.1991, SW, 1M, J. Ježek leg. \& det., slide Inv. No. 20509, NMPC, veg.: Salix, Rubus, Petasites, Mentha, Lappa, Artemisia, Urtica, Poaceae; Ondavská vrchovina hills, Kružlová (131), 14.ix.1991, SW, 1M, J. Ježek leg. \& det., slide Inv. No. 20360, NMPC, veg.: Alnus, Salix, Pinus, Pirus, Betula, Rubus, Petasites, Equisetum; Ondavská vrchovina hills, Bardejovské Kúpele (12), 15.ix.1991, SW, 1F, J. Ježek leg. \& det., slide Inv. No. 20468, NMPC, veg.: Tilia, Salix, Corylus, Frangula, Rubus, Daucaceae, Lamiaceae; Ondavská vrchovina hills, Gerlachov env. Bardejov (65), 17.ix.1991, SW, 1M, J. Ježek leg. \& det., slide Inv. No. 20375, NMPC, veg.: Alnus, Salix, Rubus, Urtica, Poaceae; Čergov Mts., Tokáreň (325), 25.ix.1992, SW, 1F, J. Ježek leg. \& det., slide Inv. No. 20444, NMPC, veg.: Acer, Picea, Fagus, Corylus, Mentha, Caltha; Čergov Mts., Olejníkov env. Majdan (213), 26.ix.1992, SW, 1M, Ježek leg. \& det., slide Inv. No. 20500, NMPC, veg.: Alnus, Salix, Corylus, Urtica; Podbeskydská vrchovina Mts., Biela Orava nr. Lokca (19), 29.vi.1992, SW, 1F, P. Chvojka leg., J. Ježek det., slide Inv. No. 20575, NMPC; Vel'ká Fatra Mts., Lubochnianska dolina valley (151), 6.xi.2006, reared, 1F, J. Lukáš leg., J. Ježek det., slide Inv. No. 20640, 
NMPC; Strážovské vrchy Mts., Diviacka Nová Ves (45), 3.vi. and 24.xi.2012, 2F, all HC, J. Oboňa leg., J. Ježek det., slides Inv. No. 20034, 20071, NMPC; Kremnické vrchy Mts., Železná Breznica env., brook (390), 30.iv.2013, SW, 1F, J. Oboňa leg., J. Ježek det., slide Inv. No. 20058, NMPC.

Comments: A cosmopolitan species, very common from lowlands to mountains.

\section{Logima erminea (Eaton, 1893)}

Published records: Hiadel'ská dolina valley (74), Hiadel'ské sedlo saddleback (75), Kochul'a (114), Liptovský Hrádok (146), Mengusovce (178), Pohronský Bukovec (235) (Ježek 1993).

Material examined: Laborecká vrchovina hills, Dedačov (44), 9.ix.1979, SW, 1M, J. Ježek leg. \& det., slide Inv. No. 20598, NMPC, veg.: Salix, Alnus, Corylus, Asarum, Urtica; Nízke Tatry Mts., Vel'ký Brankov (356), 12.ix.1985, SW, 1F, J. Ježek leg. \& det., slide Inv. No. 20487, NMPC, veg.: Picea, Rubus; Nízke Tatry Mts., Bansko, brook (nr. Liptovská Lúžna) (9), 1181 m a.s.l., 13.ix.1985, SW, 1M, J. Ježek leg. \& det., slide Inv. No. 20568, NMPC, veg.: Picea, Betula, Sambucus, Rubus, Mentha; Laborecká vrchovina hills, Malá Pol’ana (164), 10.ix.1991, SW, 1M, J. Ježek leg. \& det., slide Inv. No. 20353, NMPC, veg.: Fagus, Carpinus, Corylus, Rubus, Melampyrum, Daucaceae; Ondavská vrchovina hills, Bardejovské Kúpele (12), 15.ix.1991, SW, 1F, J. Ježek leg. \& det., slide Inv. No. 20466, NMPC, veg.: Tilia, Salix, Corylus, Frangula, Rubus, Daucaceae, Lamiaceae; Čergov Mts., Tokáreň (325), 25.ix.1992, SW, 1F, J. Ježek leg. \& det., slide Inv. No. 20443, NMPC, veg.: Acer, Picea, Fagus, Corylus, Mentha, Caltha.

Comments: Common Palaearctic species.

\section{Logima satchelli (Quate, 1955)}

Published records: Brezno (22), Il’anovo (94), Il’anovská dolina valley (95), Krámec (127), Kropácová (130), Ludrová (154), Pavčina Lehota (223), Ploštín (229), Štôla (292), Svätý Kríž (310) (Ježek 1993); Hrochotská dolina valley (87), Zadná Pol’ana (382) (Ježek 2009a).

Material examined: Košická kotlina basin, Košický Klečenov (124), 26.v.1979, SW, 1F, J. Ježek leg. \& det., slide Inv. No. 20525, NMPC, veg.: Fagus, Asarum, Trifolium, Asperula; Východoslovenská rovina plain, Vel'ký Horeš (358), 29.v.1979, SW, 1F, J. Ježek leg. \& det., slide Inv. No. 20541, NMPC, veg.: Salix, Juglans, Iris, Carex, Dactylis, Alisma; Beskydské predhorie Mts., Kamienka (106), 5.ix.1979, SW, 1M, J. Ježek leg. \& det., slide Inv. No. 20571, NMPC, veg.: Fagus, Betula, Picea, Petasites; Vihorlatské vrchy Mts., Zemplínske Hámre, brook (392), 6.ix.1979, SW, 1M, J. Ježek leg. \& det., slide Inv. No. 20339, NMPC, veg.: Salix, Alnus, Urtica; Laborecká vrchovina hills, Nižná Čabina (197), 9.ix.1979, SW, 1F, J. Ježek leg. \& det., slide Inv. No. 20308, NMPC, veg.: Salix, Alnus, Urtica, Phragmites; Popradská kotlina basin, Čierna dolina valley env. Tatranská Kotlina (36), 16.vi.1982, SW, 1F, J. Ježek leg. \& det., slide Inv. No. 19996, NMPC, veg.: Picea, Petasites, Caltha; Vysoké Tatry Mts., Zadná Kopa env. Javorina, brook (381), 26.vi.1982, SW, 1M, J. Ježek leg. \& det., slide Inv. No. 19992, NMPC, veg.: Picea, Sorbus, Petasites, Asplenium, Pinguicula; Vysoké Tatry Mts., Zadné Med'odoly (383), 27.vi.1982, SW, 1F, J. Ježek leg. \& det., slide Inv. No. 20430, NMPC, veg.: Picea, Vaccinium, Caltha; Západné Tatry Mts., Tomanová dolina valley (327), 14.viii.1983, SW, 1M, J. Ježek leg. \& det., slide Inv. No. 20553, NMPC, veg.: Picea, Petasites; Vysoké Tatry Mts., Grúnik (68), 15.viii.1983, SW, 1M, J. Ježek leg. \& det., slide Inv. No. 20503, NMPC, veg.: Picea; Vysoké Tatry Mts., Pálenica (221), 15.viii.1985, SW, 1F, J. Ježek leg. \& det., slide Inv. No. 20526, NMPC, veg.: Picea, Sorbus, Acer, Petasites; Vysoké Tatry Mts., Škaredý žl’ab trough env. Kôprová dolina valley (268), 15.viii.1983, SW, 1M, J. Ježek leg. \& det., slide Inv. No. 20516, NMPC, veg.: Picea; Východoslovenská pahorkatina hills, Michal'any (180), 23.viii.1983, SW, 1M, J. Ježek leg. \& det., slide Inv. No. 20232, NMPC, veg.: Salix, Populus, Urtica, Heracleum, Plantago, Ranunculus, Lychnis; Popradská kotlina basin, Eurocamp FICC env. Tatranská Lomnica (61), 24.viii.1983, SW, 1M, J. Ježek leg. \& det., slide Inv. No. 20590, NMPC, veg.: Picea, Pinus, Alnus, Frangula, Sorbus, Caltha, Urtica; Nízke Tatry Mts., Bansko, brook (nr. Liptovská Lúžna) (9), 1181 m a.s.l., 13.ix.1985, SW, 1M, J. Ježek leg. \& det., slide Inv. No. 20564, NMPC, veg.: Picea, Betula, Sambucus, Rubus, Mentha; Zvolenská kotlina basin, Vážna nr. Lučatín (349), 15.ix.1985, SW, 1F, J. Ježek leg. \& det., slide Inv. No. 20330, NMPC, veg.: Picea, Alnus, Petasites, Urtica; Nízke Tatry Mts., Brusno env. Brezno (24) 16.ix.1985, SW, 1M, J. Ježek leg. \& det., slide Inv. No. 20099, NMPC, veg.: Salix, Alnus, Corylus, Urtica, Asarum; Vysoké Tatry Mts., Studenovodské vodopády waterfalls (299), 11.vii.1987, SW, 1M, J. Ježek leg. \& det., slide Inv. No. 20537, NMPC, veg.: Picea, Petasites, Vaccinium; Stolické vrchy Mts., Slanská dolina valley env. Rejdová (271), 18.vii.1989, SW, 1M, J. Ježek leg. \& det., slide Inv. No. 20608, NMPC, veg.: Picea, Caltha, Mentha, Impatiens, Pteropsida; Laborecká vrchovina hills, Korejovce (117), 13.ix.1991, SW, 1M, J. Ježek leg. \& det., slide Inv. No. 20167, NMPC, veg.: Salix, Alnus, Petasites, Dipsacus, Impatiens, Poaceae; Ondavská vrchovina, Jurkova Vol'a (103), 14.ix.1991, SW, 1M, J. Ježek leg. \& det., slide Inv. No. 20508, NMPC, veg.: Salix, Rubus, Petasites, Mentha, Lappa, Artemisia, Urtica, Poaceae; Ondavská vrchovina hills, Nižná Pisaná brook (200), 14.ix.1991, SW, 1M, J. Ježek leg. \& det., slide Inv. No. 20214, NMPC, veg.: Alnus, Salix, Carpinus, Geranium, Artemisia, Urtica, Pteropsida, Poaceae; Ondavská vrchovina hills, Bardejovské Kúpele (12), 15.ix.1991, SW, 
1F, J. Ježek leg. \& det., slide Inv. No. 20467, NMPC, veg.: Tilia, Salix, Corylus, Frangula, Rubus, Daucaceae, Lamiaceae; Ondavská vrchovina hills, Šarišské Čierne (263), 15.ix.1991, SW, 1F, J. Ježek leg. \& det., slide Inv. No. 20189, NMPC, veg.: Salix, Alnus, Pirus, Frangula, Evonymus, Rubus, Urtica, Daucaceae; Spišská Magura Mts., Osturňa (220), 6.x.1991, SW, 1F, P. Chvojka leg., J. Ježek det., slide Inv. No. 20654, NMPC, Oravské Beskydy Mts., Sol'ný potok brook (276), 750 m a.s.l., 15.x.1991, SW, 1F, P. Chvojka leg., J. Ježek det., slide Inv. No. 20155, NMPC, Čergov Mts., Kyjov (134), 27.ix.1992, SW, 1M, J. Ježek leg. \& det., slide Inv. No. 20318, NMPC, veg.: Picea, Fagus, Acer, Corylus, Rubus, Urtica; Vel'ká Fatra Mts., Donovaly (54), 25.x.1994, HC, 1M, P. Lauterer leg., J. Ježek det., slide Inv. No. 20289, NMPC; Vel'ká Fatra Mts., Lubochnianska dolina valley (151), 6.xi.2006, reared, 1F, J. Lukáš leg., J. Ježek det., slide Inv. No. 20624, NMPC; Strážovské vrchy Mts., Diviacka Nová Ves (46), 21.v.2011, 3.vi., 24.xi. and 6.xii.2012, 4M, all HC, J. Oboňa leg., J. Ježek det., slides Inv. No. 20005, 20033, 20069, 20089, NMPC; Hornonitrianska kotlina basin, Vrbany env. Diviacka Nová Ves (371), 17.v.2012, SW, 1F, J. Oboňa leg., J. Ježek det., slide Inv. No. 20052, NMPC.

Comments: A common Holarctic species.

\section{Logima zetterstedti Ježek, 1983}

Published records: Lubel’a (150), Mlynica (183), Pavčina Lehota (223), Ploštín (229), Východná (372) (Ježek 1993).

Material examined: Vysoké Tatry Mts., Zadná Kopa env. Javorina brook (381), 26.vi.1982, SW, 1M, J. Ježek leg. \& det., slide Inv. No. 19994, NMPC, veg.: Picea, Sorbus, Petasites, Asplenium, Pinguicula; Liptovská kotlina basin, Pavčina Lehota nr. Liptovský Mikuláš (223), 10.vi.1987, SW, 1M, J. Ježek leg. \& det., slide Inv. No. 19952, NMPC, veg.: Salix, Rubus, Caltha, Alisma, Filipendula, Geum, Urtica; Ondavská vrchovina hills, Bukovce (25), 11.ix.1991, SW, 1M, J. Ježek leg. \& det., slide Inv. No. 20152, NMPC, veg.: Salix, Alnus, Acer, Rubus, Humulus, Petasites, Lappa, Urtica; Strážovské vrchy Mts., Laborecká vrchovina hills, Korejovce (117), 13.ix.1991, SW, 1M, J. Ježek leg. \& det., slide Inv. No. 20164, NMPC veg.: Salix, Alnus, Petasites, Dipsacus, Impatiens, Musci, Poaceae; Strážovské vrchy Mts., Diviacka Nová Ves (46), 22.iv. and 22.v.2012, SW, 2F, J. Oboňa leg., J. Ježek det., slides Inv. No. 20008, 20025, NMPC; Hornonitrianska kotlina basin, Vrbany env. Diviacka Nová Ves (371), 17.v.2012, 1F, SW, J. Oboňa leg., J. Ježek det., slide Inv. No. 20051, NMPC.

Comments: Common European and western Siberian species.

\section{Psycha Ježek, 1984}

\section{Psycha grisescens (Tonnoir, 1922)}

Published records: Demänovská dolina valley (45) (Szabó 1965a,b, Halgoš 1984); Červená Magura (32), Il'anovo (94), Ludrová (154), Patočiny (222), Pohronský Bukovec (235), Sväty Kríž (310), Štrbské Pleso (295) (Ježek 1993); Šúr (308) (Straka \& Majzlan 2010a); Šišulákov mlyn mill (266) (Straka \& Majzlan 2013).

Material examined: Vysoké Tatry Mts., Svišt'ovský potok brook (314), 25.vi.1982, SW, 1F, J. Ježek leg. \& det., slide Inv. No. 20584, NMPC, veg.: Picea; Nízke Tatry Mts., Vel'ký Brankov (356), 12.ix.1985, SW, 1F, J. Ježek leg. \& det., slide Inv. No. 20491, NMPC, veg.: Picea, Rubus, Musci; Nízke Tatry Mts., Bansko, brook (nr. Liptovská Lúžna) (9), 1181 m a.s.l., 13.ix.1985, SW, 1F, J. Ježek leg. \& det., slide Inv. No. 20565, NMPC, veg.: Picea, Betula, Sambucus, Rubus, Mentha; Nízke Tatry Mts., Liptovská Lúžna (141), brook, 13.ix.1985, SW, 2M, 1F, J. Ježek leg. \& det., slides Inv. No. 20287, 20324 and 20474, NMPC, veg.: Picea, Salix, Grossularia, Petasites, Sphagnum, Caltha, Urtica, Juncus, Mentha, Poaceae; Nízke Tatry Mts., Brusno env. Brezno (24), 16.ix.1985, SW, 1M, J. Ježek leg. \& det., slide Inv. No. 20098, NMPC, veg.: Salix, Alnus, Corylus, Urtica, Asarum; Vysoké Tatry Mts., Malý Studený potok brook nr. Nálepkova chata chalet (168), 10.vii.1987, SW, 2F, J. Ježek leg. \& det., slides Inv. No. 20425, 20332, NMPC, veg.: Picea, Petasites; Vysoké Tatry Mts., Malá Svišt'ovka env. Tatranská Lomnica (166), 9.viii.1987, SW, 1M, J. Ježek leg. \& det., slide Inv. No. 20558, NMPC, veg.: Picea, Vaccinium, Caltha, Pteropsida; Volovské vrchy Mts., Úhorná (340), 14.vii.1989, SW, 1F, J. Ježek leg. \& det., slide Inv. No. 20314, NMPC, veg.: Picea, Myosotis, Caltha, Vaccinium, Poaceae; Stolické vrchy Mts., Slanská dolina valley env. Rejdová (271), 18.vii.1989, SW, 1M, J. Ježek leg. \& det., slide Inv. No. 20606, NMPC, veg.: Picea, Caltha, Mentha, Impatiens, Pteropsida; Ondavská vrchovina hills, Bukovce (25), 11.ix.1991, SW, 1M, J. Ježek leg. \& det., slide Inv. No. 20153, NMPC, veg.: Salix, Alnus, Acer, Rubus, Humulus, Petasites, Lappa, Urtica; Ondavská vrchovina hills, Nižná Pisaná, brook (200), 14.ix.1991, SW, 1M, J. Ježek leg. \& det., slide Inv. No. 20212, NMPC, veg.: Alnus, Salix, Carpinus, Geranium, Artemisia, Urtica, Pteropsida, Poaceae; Spišská Magura Mts., Osturňa (220), 6.x.1991, SW, 1M, P. Chvojka leg., J. Ježek det., slide Inv. No. 20653, NMPC, Chočské vrchy Mts., Valaská Dubová, well (347), 4.vii.1992, SW, 1M, P. Chvojka leg., J. Ježek det., slide Inv. No. 20198, NMPC; Spišsko-šarišské medzihorie, Sabinov (262), 24.ix.1992, SW, 1F, J. Ježek leg. \& det., slide Inv. No. 20249, NMPC, veg.: Salix, Corylus, Mentha, Impatiens; Čergov Mts., Tokáreň (325), 25.ix.1992, SW, 1F, J. Ježek leg. \& det., slide Inv. No. 20445, NMPC, veg.: Acer, Picea, Fagus, Corylus, Mentha, Caltha; Čergov Mts., Hromacký vrch env. Livov (88), 830 m a.s.l., 26.ix.1992, 
SW, 1M, J. Ježek leg. \& det., slide Inv. No. 20579, NMPC, veg.: Fagus, Picea, Pinus, Abies, Betula, Mentha, Poaceae; Spišsko-šarišské medzihorie Imts., Stará Lubovňa (284), 28.ix.1992, SW, 1M, J. Ježek leg. \& det., slide Inv. No. 20659, NMPC, veg.: Alnus, Salix, Prunus, Corylus, Ribes, Colchicum, Geranium, Mentha, Urtica.

Comments: A European and North African species.

\section{Psychoda Latreille, 1796}

\section{Psychoda alticola Vaillant, 1973}

(syn. dolomitica Salamanna \& Sarà, 1980 - more see Kvifte \& Andersen 2012)

Material examined: Laborecká vrchovina hills, Dedačov (44), 9.ix.1979, SW, 1M, J. Ježek leg. \& det., slide Inv. No. 20599, NMPC, veg.: Salix, Alnus, Corylus, Asarum, Urtica; Vysoké Tatry Mts., Zadná Kopa env. Javorina brook (381), 26.vi.1982, SW, 1M, J. Ježek leg. \& det., slide Inv. No. 19995, NMPC, veg.: Picea, Sorbus, Petasites, Asplenium, Pinguicula; Vysoké Tatry Mts., Grúnik (68), 15.viii.1983, SW, 1M, J. Ježek leg. \& det., slide Inv. No. 20502, NMPC, veg.: Picea; Vysoké Tatry Mts., Malý Studený potok brook nr. Nálepkova chata chalet (168), 10.vii.1987, SW, 1M, J. Ježek leg. \& det., slide Inv. No. 20331, NMPC, veg.: Picea, Petasites; Vysoké Tatry Mts., Studenovodské vodopády waterfalls (299), 11.vii.1987, SW, 1M, J. Ježek leg. \& det., slide Inv. No. 20536, NMPC, veg.: Picea, Petasites, Vaccinium; Vysoké Tatry Mts., Malá Svišt'ovka env. Tatranská Lomnica (166), 9.viii.1987, SW, 1M, J. Ježek leg. \& det., slide Inv. No. 20583, NMPC, veg.: Picea, Vaccinium, Caltha, Pteropsida; Volovské vrchy Mts., Okrúhly chrbát saddle env. Úhorná (211), 14.vii.1989, SW, 1F, J. Ježek leg. \& det., slide Inv. No. 20594, NMPC, veg.: Picea, Sphagnum, Myosotis, Poaceae; Stolické vrchy Mts., Slanská dolina valley env. Rejdová (271), 17.vii.1989, SW, 1F, J. Ježek leg. \& det., slide Inv. No. 20663, NMPC, veg. Picea, Alnus, Rubus, Filipendula, Scirpus, Pteropsida; Revúcka vrchovina Mts., Rejdová (250), 18.vii.1989, SW, 2F, J. Ježek leg. \& det., slides Inv. No. 20455, 20480, NMPC, veg.: Picea, Fagus, Salix, Sambucus, Caltha, Scirpus, Daucaceae; Podtatranská brázda furrow, Ždiar (389), 26.ix.-2.x.1993, YPT, 1M, P. Chvojka leg., J. Ježek det., slide Inv. No. 20484, NMPC.

Comments: All mentioned localities see in Map 5. The species was previously recorded from arctic-alpine localities in Austria, Czech Republic, France, Finland, Germany, Italy, Norway and Rumania (Wagner 1977, Salamanna \& Sarà 1980, Ježek 2006b, Kvifte \& Andersen 2012). New species for Slovakia.

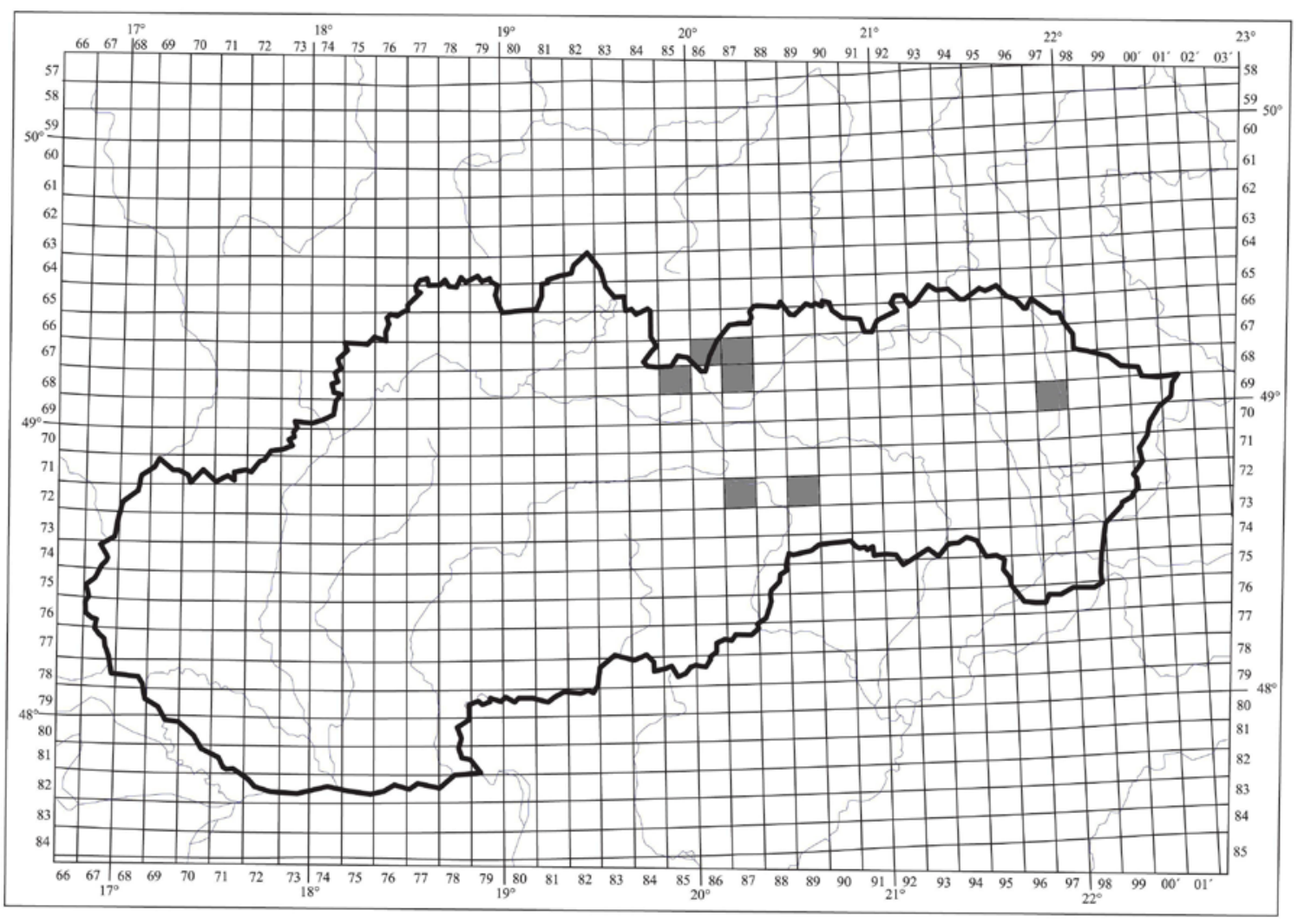

Map 5: Map showing records of Psychoda alticola Vaillant, 1973 from Slovakia (DFS: 6786 - Zadná Kopa, 6787 - Ždiar, 6885 - Grúnik, 6887 - Malá Svišt’ovka, Malý Studený potok, Studenovodské vodopády waterfalls, 6997 - Dedačov, 7287 - Rejdová, Slanská dolina valley, 7289 - Okrúhly chrbát ridge). 


\section{Psychoda phalaenoides (Linnaeus, 1758)}

Published records: Oravský Podzámok (219) (Szabó 1965a); Oravský Podzámok (219), Demänovská dolina valley (45) (Szabó 1965b); Bratislava (19), Malacky (167), Nová Baňa (204) (Halgoš 1973); Bratislava (19), Demänovská dolina valley (45), Malacky (167), Nitra - Zobor (194), Nová Baňa (204), Oravksy Podzámok (219) (Halgoš 1984); Brezno (22), Črmné (42), Dúbrava (56), Il’anovo (94), Il’anovská dolina valley (95), Ludrová (153), Mengusovce (178), Patočiny (222), Pohronský Bukovec (235), Štôla (292) (Ježek 1993); Dunaj river basin (60), Hron river basin (89), lower Váh river basin (149), Morava river basin (186), Poprad - Dunajec river basin (236), upper Váh river basin (345) (larvae, Bulánková 2003); Hrochotská dolina valley (87), Hrončecký grúň slope (90), Kyslinky (136), Zadná Pol’ana (382) (Ježek 2009a); Dubová (55), Súčanka (303) (Straka 2009); Šúr (309) (Straka \& Majzlan 2010a).

Material examined: Košická kotlina basin, Košický Klečenov (124), 26.v.1979, SW, 1M, J. Ježek leg. \& det., slide Inv. No. 20522, NMPC, veg.: Fagus, Asarum, Trifolium, Asperula; Bukovské vrchy Mts., Kolbasov (116), 6.ix.1979, SW, 1F, J. Ježek leg. \& det., slide Inv. No. 20613, NMPC, veg.: Sambucus, Salix, Alnus, Urtica; Bukovské vrchy Mts., Uličské Krivé (343), 7.ix.1979, SW, 1M, J. Ježek leg. \& det., slide Inv. No. 20618, NMPC, veg.: Alnus, Salix, Scirpus; Bukovské vrchy Mts., Zboj (386), 7.ix.1979, SW, 1M, J. Ježek leg. \& det., slide Inv. No. 20529, NMPC, veg.: Alnus, Corylus, Humulus, Mentha; Laborecká vrchovina hills, Nižná Čabina (197), 9.ix.1979, SW, 1F, J. Ježek leg. \& det., slide Inv. No. 20309, NMPC, veg.: Salix, Alnus, Urtica, Phragmites; Laborecká vrchovina hills, Volica (367), 9.ix.1979, SW, 1M, J. Ježek leg. \& det., slide Inv. No. 20651, NMPC, veg.: Salix, Urtica; Vysoké Tatry Mts., Zadná Kopa env. Javorina, brook (381), 26.vi.1982, SW, 1F, J. Ježek leg. \& det., slide Inv. No. 19993, NMPC, veg.: Picea, Sorbus, Petasites, Asplenium, Pinguicula; Nízke Tatry Mts., Vel'ký Brankov (356), 12.ix.1985, SW, 1F, J. Ježek leg. \& det., slide Inv. No. 20488, NMPC, veg.: Picea, Rubus; Nízke Tatry Mts., Bansko, brook (nr. Liptovská Lúžna) (9), 1181 m a.s.l., 13.ix.1985, SW, 1M, J. Ježek leg. \& det., slide Inv. No. 20561, NMPC, veg.: Picea, Betula, Sambucus, Rubus, Mentha; Nízke Tatry Mts., Liptovská Lúžna (141), 13.ix.1985, SW, 2M, J. Ježek leg. \& det., slides Inv. No. 20471, 20283, NMPC, veg.: Salix, Grossularia, Petasites, Mentha, Urtica; Nízke Tatry Mts., Brusno env. Brezno (24), 16.ix.1985, SW, 1M, J. Ježek leg. \& det., slide Inv. No. 20093, NMPC, veg.: Salix, Alnus, Corylus, Urtica, Asarum; Liptovská kotlina basin, Pavčina Lehota nr. Liptovský Mikuláš (223), 10.vi.1987, SW, 1M, J. Ježek leg. \& det., slide Inv. No. 19941, NMPC, veg.: Salix, Rubus, Caltha, Alisma, Filipendula, Geum, Urtica; Volovské vrchy Mts., Úhorná (340), 14.vii.1989, SW, 1F, J. Ježek leg. \& det., slide Inv. No. 20313, NMPC, veg.: Picea, Myosotis, Caltha, Vaccinium, Poaceae; Stolické vrchy Mts., Slanská dolina valley env. Rejdová (271), 18.vii.1989, SW, 1M, J. Ježek leg. \& det., slide Inv. No. 20602, NMPC, veg.: Picea, Caltha, Mentha, Impatiens, Pteropsida; Popradská kotlina basin, Červený potok brook - Nová Lesná (34), 22.vii.1990, SW, 1M, P. Chvojka leg., J. Ježek det., slide Inv. No. 20412, NMPC; Laborecká vrchovina hills, Krásny Brod env. Medzilaborce, brook, well (128), 10.ix.1991, SW, 1M, J. Ježek leg. \& det., slide Inv. No. 20110, NMPC, veg.: Alnus, Salix, Corylus, Rubus, Caltha, Mentha, Carex, Dipsacus, Musci; Ondavská vrchovina hills, Bukovce (25), 11.ix.1991, SW, 1M, J. Ježek leg. \& det., slide Inv. No. 20149, NMPC, veg.: Salix, Alnus, Acer, Rubus, Humulus, Petasites, Lappa, Urtica; Ondavská vrchovina hills, Mrázovce (189), 11.ix.1991, SW, 1F, J. Ježek leg. \& det., slide Inv. No. 20180, NMPC, veg.: Salix, Alnus, Pirus, Frangula, Evonymus, Rubus, Urtica, Daucaceae; Ondavská vrchovina hills, Krajné Čierno (125), 12.ix.1991, SW, 1F, J. Ježek leg. \& det., slide Inv. No. 20439, NMPC, veg.: Fagus, Carpinus, Betula, Salix, Petasites, Juncus, Impatiens, Poaceae; Ondavská vrchovina hills, Nižná Pisaná brook (200), 14.ix.1991, SW, 1M, J. Ježek leg. \& det., slide Inv. No. 20207, NMPC, veg.: Alnus, Salix, Carpinus, Geranium, Artemisia, Urtica, Pteropsida, Poaceae; Ondavská vrchovina hills, Bardejovské Kúpele (12), 15.ix.1991, SW, 1F, J. Ježek leg. \& det., slide Inv. No. 20464, NMPC, veg.: Tilia, Salix, Corylus, Frangula, Rubus, Daucaceae, Lamiaceae; Ondavská vrchovina hills, Gerlachov env. Bardejov (65), 17.ix.1991, SW, 1M, J. Ježek leg. \& det., slide Inv. No. 20373, NMPC, veg.: Alnus, Salix, Rubus, Urtica, Poaceae; Podtatranská brázda furrow, Ždiar (389), 26.ix.-2.x.1993, YPT, 1M, P. Chvojka leg., J. Ježek det., slide Inv. No. 20481, NMPC; Chočské vrchy Mts., Valaská Dubová, well (347), 6.vii.1994, SW, 1F, 1M, P. Lauterer leg., J. Ježek det., slides Inv. No. 20459, 20585, NMPC; Hornonitrianska kotlina basin, Liešt'any, meanders of a brook (140), 23.v.2013, SW, 1M, J. Oboňa leg., J. Ježek det., slide Inv. No. 20075, NMPC.

Comments: A very common Holarctic polyvoltine species.

\section{Psychoda uniformata Haseman, 1907}

Materiala examined: Východoslovenská rovina plain, Ptrukša (242), 7.ix.1979, SW, 1F, J. Burda leg., J. Ježek det., slide Inv. No. 20497, NMPC; Bukovské vrchy Mts., Ulič (341), 7.ix.1979, SW, 1F, J. Ježek leg. \& det., slide Inv. No. 20496, NMPC, veg.: Alnus, Mentha, Scirpus; Bukovské vrchy Mts., Uličské Krivé (343), 7.ix.1979, SW, 1M, J. Ježek leg. \& det., slide Inv. No. 20620, NMPC, veg.: Alnus, Salix, Scirpus; Bukovské vrchy Mts., Zboj (386), 7.ix.1979, SW, 1F, J. Ježek leg. \& det., slide Inv. No. 20531, NMPC, veg.: Alnus, Corylus, Humulus, Mentha; Laborecká vrchovina hills, Hankovce (70), 9.ix.1979, SW, 1F, J. Ježek leg. \& det., slide Inv. No. 20514, NMPC, veg.: Salix, Sambucus, Cornus, Urtica; Ondavská vrchovina hills, Kružlová (131), 
14.ix.1991, SW, 1F, J. Ježek leg. \& det., slide Inv. No. 20359, NMPC, veg.: Alnus, Salix, Pinus, Pirus, Betula, Rubus, Petasites, Equisetum; Ondavská vrchovina hills, Nižná Pisaná, brook (200), 14.ix.1991, SW, 1F, J. Ježek leg. \& det., slide Inv. No. 20211, NMPC, veg.: Alnus, Salix, Carpinus, Geranium, Artemisia, Urtica, Pteropsida, Poaceae; Ondavská vrchovina, Šarišské Čierne (263), 15.ix.1991, SW, 2F, J. Ježek leg. \& det., slides Inv. No. 20187, 20188, NMPC, veg.: Salix, Alnus, Pirus, Frangula, Evonymus, Rubus, Urtica, Daucaceae.

Comments: Above mentioned localities of this species see Map 6. Distribution: A Holarctic species, recorded from Austria, Czech Republic, Italy, Slovenia, Greece, Turkey, Iran, Israel, Mongolia, and the USA (Ježek \& Omelková 2012). New species for Slovakia.

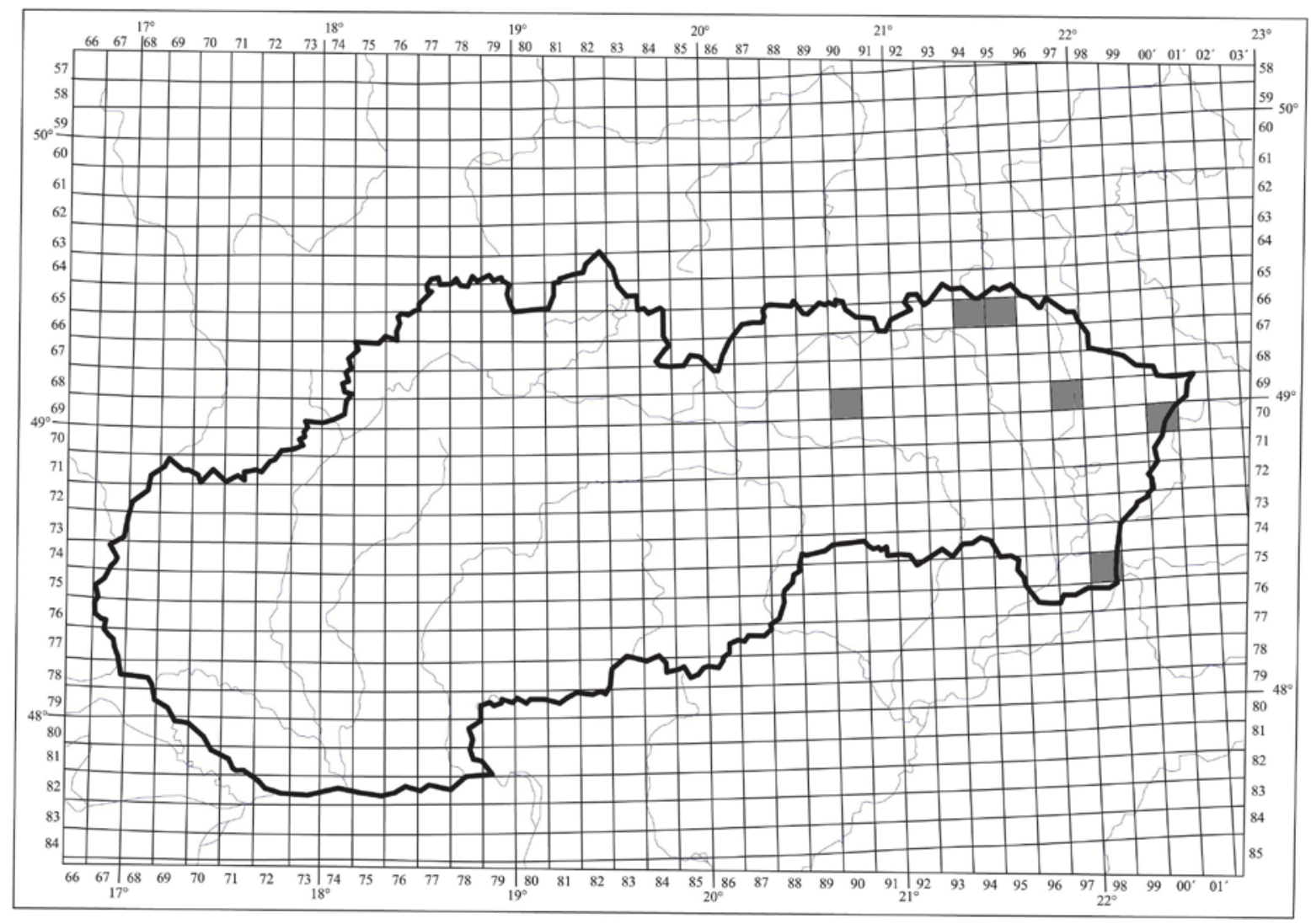

Map 6: Map showing records of Psychoda uniformata Haseman, 1907 from Slovakia (DFS: 6694 - Šarišské Čierne, 6695 - Kružlová, Nižná Pisaná, 6900 - Zboj, 6997 - Hankovce, 7000 - Ulič, Uličské Krivé, 7598 Ptrukša).

\section{Psychodocha Ježek, 1984}

\section{Psychodocha cinerea (Banks, 1894)}

Published records: Bratislava (19) (Halgoš 1984); Suchý vrch Mt. (306) (Ježek 1990b); Hradište (83), Il’anovská dolina valley (95), Kochul'a (114), Kršahy, Litvorový Žl’ab (147), Ludrová (153), Sopotnická dolina valley (278), Tokárensky potok brook (326) (Ježek 1993); Zbojnícka jaskyňa cave (387) (Košel \& Horváth 1996); Hron river basin (89), Poprad - Dunajec river basin (236), upper Váh river basin (345) (larvae, Bulánková 2003); Nad Šenkárkou (193) (Straka \& Majzlan 2006); Diviacka Nová Ves (45), Vrbany (371) (Oboňa a Ježek 2012b).

Material examined: Východoslovenská rovina plain, Kapušianske Kl’ačany (107), 29.v.1979, SW, 1M, J. Ježek leg. \& det., slide Inv. No. 20589, NMPC, veg.: Quercus, Fraxinus, Populus, Salix, Iris; Vysoké Tatry Mts., Škaredý žl'ab trough env. Kôprová dolina valley (268), 15.viii.1983, SW, 1M, J. Ježek leg. \& det., slide Inv. No. 20517, NMPC, veg.: Picea; Nízke Tatry Mts., Liptovská Lúžna (141), 13.ix.1985, SW, 1F, J. Ježek leg. \& det., slide Inv. No. 20475, NMPC, veg.: Picea, Mentha, Urtica; Ondavská vrchovina hills, Bukovce (25), 11.ix.1991, SW, 1M, J. Ježek leg. \& det., slide Inv. No. 20151, NMPC, veg.: Salix, Alnus, Acer, Rubus, Humulus, Petasites, Lappa, Urtica; Ondavská vrchovina hills, Bardejovské Kúpele (12), 15.ix.1991, SW, 1F, J. Ježek leg. \& det., slide Inv. No. 20463, NMPC, veg.: Tilia, Salix, Corylus, Frangula, Rubus, Daucaceae, 
Lamiaceae; Belianske Tatry Mts., Alabastrová jaskyňa cave (1), 10.viii.2000, HC, 1M, TE, V. Košel leg., J. Ježek det., slide Inv. No. 9851, NMPC; Vel'ká Fatra Mts., Lubochnianska dolina valley (151), 6.xi.2006, reared, 1F, J. Lukáš leg., J. Ježek det., slide Inv. No. 20625, NMPC; Malé Karpaty Mts., Mokrý potok brook (184), 210 m a.s.l., 25.viii.2011, SW, 1F, J. Lukáš leg., J. Ježek det., slide Inv. No. 20646, NMPC; Strážovské vrchy Mts., Diviacka Nová Ves (46), 3.vi., 15.viii., 3.ix. and 24.xi.2012, 1M, 3F, all HC, J. Oboňa leg., J. Ježek det., slides Inv. No. 20035, 20022, 20036 and 20070, NMPC; Zvolenská kotlina basin, Zvolen (396), 297 m a.s.l., 7.viii.2012, 1F, HC, J. Oboňa leg., J. Ježek det., slide Inv. No. 20006, NMPC; Považské podolie basin, Opatovské jazero lake (215), 20.iv.2013, 1F, SW, J. Oboňa \& M. Svitok leg., J. Ježek det., slide Inv. No. 20087, NMPC; Hornonitrianska kotlina basin, Vrbany env. Diviacka Nová Ves (371), 28.viii.2013, 1M, HC from Quercus tree holes, J. Oboňa leg., J. Ježek det., slide Inv. No. 20248, NMPC.

Comments: A very common cosmopolitan species.

\section{Psychodocha gemina (Eaton, 1904)}

Published records: Baláže (6), Brtkovica (23), Bukovec (26), Bukovecká dolina valley (28), Črmné (42), Hiadel'ská dolina valley (74), Il’anovo (94), Kežmarské Žl’aby (111), Kochul'a (114), Krámec (127), Liptovská Porúbka (142), Liptovský Hrádok (146), Ludrová (153), Mengusovce (178), Patočiny (222), Pohronský Bukovec (235), Sväty Kríž (310), Tri studničky well (332) (Ježek 1993); Hučava stream (93), Mičová stream (181) (larvae, Bulánková 1999); Hučava stream (93), Mičová stream (181) (larvae, Bitušík et al. 2000); Gidra stream (67) (larvae, Bulánková et al. 2001, 2002); Hron river basin (89), Poprad - Dunajec river basin (236), upper Váh river basin (345) (larvae, Bulánková 2003); Hrochotská dolina valley (87), Zadná Pol’ana (382) (Ježek 2009a).

Material examined: Východoslovenská pahorkatina hills, Zámutov env. Vranov nad Toplou, brook (384), 24.v.1979, SW, 1F, J. Ježek leg. \& det., slide Inv. No. 20586, NMPC, veg.: Fagus, Betula, Mentha, Urtica; Košická kotlina basin, Košický Klečenov (124), 26.v.1979, SW, 1F, J. Ježek leg. \& det., slide Inv. No. 20523, NMPC, veg.: Fagus, Asarum, Trifolium, Asperula; Beskydské predhorie Mts., Kamienka (106), 5.ix.1979, SW, 1F, J. Ježek leg. \& det., slide Inv. No. 20572, NMPC, veg.: Fagus, Betula, Picea, Petasites; Bukovské vrchy Mts., Ulič (342), 7.ix.1979, SW, 1M, J. Ježek leg. \& det., slide Inv. No. 20495, NMPC, veg.: Alnus, Mentha, Scirpus; Bukovské vrchy Mts., Uličské Krivé (343), 7.ix.1979, SW, 1M, J. Ježek leg. \& det., slide Inv. No. 20616, NMPC, veg.: Alnus, Salix, Scirpus; Veporské vrchy Mts., Príslop (240), 8.ix.1979, SW, 1M, J. Ježek leg. \& det., slide Inv. No. 20262, NMPC, veg.: Alnus, Juncus, Mentha, Carex; Laborecká vrchovina hills, Hankovce (70), 9.ix.1979, SW, 1M, J. Ježek leg. \& det., slide Inv. No. 20512, NMPC, veg.: Salix, Sambucus, Cornus, Urtica; Laborecká vrchovina hills, Nižná Čabina (197), 9.ix.1979, SW, 1F, J. Ježek leg. \& det., slide Inv. No. 20311, NMPC, veg.: Salix, Alnus, Urtica, Phragmites; Popradská kotlina basin, Čierna dolina valley env. Tatranská Kotlina (36), 16.vi.1982, SW, 1M, J. Ježek leg. \& det., slide Inv. No. 19997, NMPC, veg.: Picea, Petasites, Caltha; Vysoké Tatry Mts., Svišt'ovský potok brook (314), 25.vi.1982, SW, 1F, J. Ježek leg. \& det., slide Inv. No. 20557, NMPC, veg.: Picea; Vysoké Tatry Mts., Zadné Med'odoly (383), 27.vi.1982, SW, 1F, J. Ježek leg. \& det., slide Inv. No. 20429, NMPC, veg.: Picea, Vaccinium, Caltha; Popradská kotlina basin, Tatranská Polianka (320), 21.viii.1983, SW, 1M, J. Ježek leg. \& det., slide Inv. No. 20427, NMPC, veg.: Picea, Alnus, Rubus, Caltha, Lysimachia, Filipendula, Urtica; Nízke Tatry Mts., Vel'ký Brankov (356), 12.ix.1985, SW, 1F, J. Ježek leg. \& det., slide Inv. No. 20485, NMPC, veg.: Picea, Rubus, Musci; Nízke Tatry Mts., Bansko, brook (nr. Liptovská Lúžna) (9), 1181 m a.s.l., 13.ix.1985, SW, 1F, J. Ježek leg. \& det., slide Inv. No. 20560, NMPC, veg.: Picea, Betula, Sambucus, Rubus, Mentha; Nízke Tatry Mts., Liptovská Lúžna (141), 13.ix.1985, SW, 2F, J. Ježek leg. \& det., slides Inv. No. 20472, 20285, NMPC, veg.: Salix, Grossularia, Petasites, Mentha, Urtica; Zvolenská kotlina basin, Vážna nr. Lučatín (350), 15.ix.1985, SW, 1F, J. Ježek leg. \& det., slide Inv. No. 20327, NMPC, veg.: Picea, Alnus, Petasites, Urtica; Nízke Tatry Mts., Brusno env. Brezno (24), 16.ix.1985, SW, 1M, J. Ježek leg. \& det., slide Inv. No. 20096, NMPC, veg.: Salix, Alnus, Corylus, Urtica, Asarum; Nízke Tatry Mts., Čierny Diel env. Ráztoka (38), 1144 m a.s.l., 18.ix.1985, SW, 1M, J. Ježek leg. \& det., slide Inv. No. 20408, NMPC, veg.: Picea, Salix, Acer, Corylus, Eupatorium, Caltha, Pinguicula; Nízke Tatry Mts., Stanišovské sedlo saddleback nr. Liptovská Porúbka (283), 8.ix.1986, SW, 1M, J. Ježek leg. \& det., slide Inv. No. 19974, NMPC; Rožňavská kotlina basin, Rožňava (257), 13.vii.1989, SW, 1M, J. Ježek leg. \& det., slide Inv. No. 20405, NMPC, veg.: Alnus, Carpinus, Juncus, Impatiens, Pteropsida; Revúcka vrchovina Mts., Rejdová (250), 18.vii.1989, SW, 2M, J. Ježek leg. \& det., slides Inv. No. 20479, 20604, NMPC, veg.: Picea, Fagus, Salix, Sambucus, Caltha, Scirpus, Daucaceae; Stolické vrchy Mts., Slanská dolina valley env. Rejdová (271), 18.vii.1989, SW, 1M, J. Ježek leg. \& det., slide Inv. No. 20660, NMPC, veg.: Picea, Caltha, Mentha, Impatiens, Pteropsida; Stolické vrchy Mts., Muránska Zdychava (192), 19.vii.1989, SW, 1M, J. Ježek leg. \& det., slide Inv. No. 20141, NMPC, veg.: Alnus, Rubus, Filipendula, Impatiens, Caltha, Pteropsida; Laborecká vrchovina hills, Krásny Brod env. Medzilaborce, brook, well (128), 10.ix.1991, SW, 1M, J. Ježek leg. \& det., slide Inv. No. 20117, NMPC, veg.: Alnus, Salix, Corylus, Rubus, Caltha, Mentha, Carex, Dipsacus, Musci; Laborecká vrchovina hills, Malá Pol’ana (164), 10.ix.1991, SW, 1M, J. Ježek leg. \& det., slide Inv. No. 20351, NMPC, veg.: Fagus, Carpinus, Corylus, Rubus, Melampyrum, Daucaceae; Ondavská vrchovina hills, Makovce (161), 10.ix.1991, SW, 1M, J. Ježek leg. \& det., slide Inv. No. 20451, NMPC, veg.: Alnus, Salix, 
Rubus, Polygonum, Lappa, Urtica, Poaceae; Ondavská vrchovina hills, Mrázovce (189), 11.ix.1991, SW, 1F, J. Ježek leg. \& det., slide Inv. No. 20179, NMPC, veg.: Salix, Alnus, Pirus, Frangula, Evonymus, Rubus, Urtica, Daucaceae; Ondavská vrchovina hills, Krajné Čierno (125), 12.ix.1991, SW, 1F, J. Ježek leg. \& det., slide Inv. No. 20438, NMPC, veg.: Fagus, Carpinus, Betula, Salix, Petasites, Juncus, Impatiens, Poaceae; Ondavská vrchovina hills, Rakovčík (246), 12.ix.1991, SW, 1M, J. Ježek leg. \& det., slide Inv. No. 20294, NMPC, veg.: Alnus, Prunus, Corylus, Sambucus Salix, Cirsium, Poaceae, Daucaceae; Ondavská vrchovina hills, Svidník (313), 12.ix.1991, SW, 1M, J. Ježek leg. \& det., slide Inv. No. 20456, NMPC, veg.: Salix, Alnus, Petasites, Symphytum, Mentha, Impatiens, Poaceae; Laborecká vrchovina hills, Korejovce (117), 13.ix.1991, SW, 1M, Ježek leg. \& det., slide Inv. No. 20166, NMPC, veg.: Salix, Alnus, Petasites, Dipsacus, Impatiens, Musci, Poaceae; Ondavská vrchovina hills, Jurkova Vol'a (103), 14.ix.1991, SW, 1M, J. Ježek leg. \& det., slide Inv. No. 20505, NMPC, veg.: Salix, Rubus, Petasites, Mentha, Lappa, Artemisia, Urtica, Poaceae; Ondavská vrchovina hills, Kružlová (131), 14.ix.1991, SW, 1M, J. Ježek leg. \& det., slide Inv. No. 20358, NMPC, veg.: Alnus, Salix, Pinus, Pirus, Betula, Rubus, Petasites, Equisetum; Ondavská vrchovina hills, Nižná Pisaná, brook (200), 14.ix.1991, SW, 1M, J. Ježek leg. \& det., slide Inv. No. 20210, NMPC, veg.: Alnus, Salix, Carpinus, Geranium, Artemisia, Urtica, Pteropsida, Poaceae; Ondavská vrchovina hills, Bardejovské Kúpele (12), 15.ix.1991, SW, 1F, J. Ježek leg. \& det., slide Inv. No. 20462, NMPC, veg.: Tilia, Salix, Corylus, Frangula, Rubus, Daucaceae, Lamiaceae; Ondavská vrchovina hills, Gerlachov env. Bardejov (65), 17.ix.1991, SW, 1M, J. Ježek leg. \& det., slide Inv. No. 20374, NMPC, veg.: Alnus, Salix, Rubus, Urtica, Poaceae; Čergov Mts., Tokáreň (325), 25.ix.1992, SW, 1F, J. Ježek leg. \& det., slide Inv. No. 20442, NMPC, veg.: Acer, Picea, Fagus, Corylus, Mentha, Caltha; Čergov Mts., Hromacký vrch env. Livov (88), 830 m a.s.l., 26.ix.1992, SW, 1F, J. Ježek leg. \& det., slide Inv. No. 20578, NMPC, veg.: Fagus, Picea, Pinus, Abies, Betula, Mentha, Poaceae; Čergov Mts., Olejníkov (214), 26.ix.1992, SW, 1M, J. Ježek leg. \& det., slide Inv. No. 20390, NMPC, veg.: Alnus, Rubus, Petasites, Caltha, Urtica, Mentha, Marchantiopsida; Spišsko-šarišské medzihorie Imts., Stará Lubovňa (284), 28.ix.1992, SW, 1M, J. Ježek leg. \& det., slide Inv. No. 20658, NMPC, Alnus, Salix, Prunus, Corylus, Ribes, Colchicum, Geranium, Mentha, Urtica; Muránska planina Mts., Hrdzavá (84), 19.vii.1999, SW, 1M, P. Chvojka leg., J. Ježek det., slide Inv. No. 20554, NMPC; Bukovské vrchy Mts., Jarabá skala rock (99), 12.viii.2003, SW, 1M, J. Ševčík leg., J. Ježek det., slide Inv. No. 12022, NMPC; Laborecká vrchovina hills, Starina env., brook (288), 13.viii.2003, SW, 1M, J. Ševčík leg., J. Ježek det., slide Inv. No. 12025, NMPC, veg. Alnus, Fagus, Carpinus; Malé Karpaty Mts., Mokrý potok brook (184), 210 m a.s.l., 25.viii.2011, SW, 1F, J. Lukáš leg., J. Ježek det., slide Inv. No. 20645, NMPC; Zvolenská kotlina basin, Zvolen (396), 297 m a.s.l., 7.8.2012, 1F, HC, J. Oboňa leg., J. Ježek det., slide Inv. No. 20007, NMPC; Strážovské vrchy Mts., Trstenec well env. Diviacka Nová Ves (334), 6.v. and 1.vii.2013, 1F, 1M, SW, J. Oboňa leg., J. Ježek det., slide Inv. No. 20066, 20225, NMPC.

Comments: A common European species.

\section{Psychodula Ježek, 1984}

\section{Psychodula minuta (Banks, 1894)}

Published records: Hiadel'ské sedlo saddleback (75) (Ježek 1990b); Brtkovica (23), Ráztoka (249) (Ježek 1993); Nad Šenkárkou (193) (Straka \& Majzlan 2006); Čierna Lehota (37) (Straka \& Majzlan 2007); Zadná Pol’ana (382) (Ježek 2009a); Šúr (309) (Straka \& Majzlan 2010a); Diviacka Nová Ves (46), Vrbany (371) (Oboňa a Ježek 2012b).

Material examined: Východoslovenská rovina plain, Vel'ký Horeš (358), 29.v.1979, SW, 1F, J. Ježek leg. \& det., slide Inv. No. 20538, NMPC, veg.: Salix, Juglans, Iris, Carex, Dactylis, Alisma; Nízke Tatry Mts., Bansko, brook (nr. Liptovská Lúžna) (9), 1181 m a.s.l., 13.ix.1985, SW, 1M, J. Ježek leg. \& det., slide Inv. No. 20567, NMPC, veg.: Picea, Betula, Sambucus, Rubus, Mentha; Zvolenská kotlina basin, Vážna nr. Lučatín (350), 15.ix.1985, SW, 1M, J. Ježek leg. \& det., slide Inv. No. 20328, NMPC, veg.: Picea, Alnus, Petasites, Urtica; Stolické vrchy Mts., Slanská dolina valley env. Rejdová (271), 18.vii.1989, SW, 1M, J. Ježek leg. \& det., slide Inv. No. 20605, NMPC, veg.: Picea, Caltha, Mentha, Impatiens, Pteropsida; Spišsko-šarišské medzihorie Imts., Stará Lubovňa (284), 28.ix.1992, SW, 1F, J. Ježek leg. \& det., slide Inv. No. 20657, NMPC, Alnus, Salix, Prunus, Corylus, Ribes, Colchicum, Geranium, Mentha, Urtica; Podtatranská brázda furrow, Ždiar (389), 26.ix.-2.x.1993, YPT, 1M, P. Chvojka leg., J. Ježek det., slide Inv. No. 20482, NMPC; Slovenský raj, Stratená jaskyňa cave (293), 5.viii.1994, HC, 1M, V. Košel leg., J. Ježek det., slide Inv. No. 19964, NMPC; Strážovské vrchy Mts., Diviacka Nová Ves (46), 25.v.2012, 1F, HC, J. Oboňa leg., J. Ježek det., slide Inv. No. 20029, NMPC.

Comments: A generally common Holarctic species. 


\title{
Psychomora Ježek, 1984
}

\section{Psychomora mycophila (Vaillant, 1988)}

\author{
(syn. vanharai Ježek, 1995)
}

Published records: Nová Sedlica (208) (Ševčík 2004, 2010); Hrochotská dolina valley (87) (Ježek 2009a).

Comments: Rather a rare European species associated with fungi.

\section{Psychomora trinodulosa (Tonnoir, 1922)}

Published records: Sopotnická dolina valley (278) (Ježek 1990b); Ludrová (153), Mlynica (183), Patočiny (222) (Ježek 1993).

Material examined: Východoslovenská rovina plain, Vel’ký Horeš (358), 29.v.1979, SW, 1F, J. Ježek leg. \& det., slide Inv. No. 20543, NMPC, veg.: Salix, Juglans, Iris, Carex, Dactylis, Alisma; Bukovské vrchy Mts., Kolbasov (116), 6.ix.1979, SW, 1M, J. Ježek leg. \& det., slide Inv. No. 20611, NMPC, veg.: Sambucus, Salix, Alnus, Urtica; Vihorlatské vrchy Mts., Zemplínske Hámre, brook (392), 6.ix.1979, SW, 1M, J. Ježek leg. \& det., slide Inv. No. 20340, NMPC, veg.: Salix, Alnus, Urtica; Bukovské vrchy Mts., Uličské Krivé (343), 7.ix.1979, SW, 1M, J. Ježek leg. \& det., slide Inv. No. 20615, NMPC, veg.: Alnus, Salix, Scirpus; Bukovské vrchy Mts., Ulič (342), 7.ix.1979, SW, 1M, J. Ježek leg. \& det., slide Inv. No. 20494, NMPC, veg.: Alnus, Mentha, Scirpus; Bukovské vrchy Mts., Zboj (386), 7.ix.1979, SW, 1M, J. Ježek leg. \& det., slide Inv. No. 20530, NMPC, veg.: Alnus, Corylus, Humulus, Mentha; Laborecká vrchovina hills, Nižná Čabina (197), 9.ix.1979, SW, 1M, J. Ježek leg. \& det., slide Inv. No. 20310, NMPC, veg.: Salix, Alnus, Urtica, Phragmites; Bukovské vrchy Mts., Topol’a (328), 8.ix.1979, SW, 1M, J. Ježek leg. \& det., slide Inv. No. 20419, NMPC, veg.: Alnus, Corylus, Urtica; Laborecká vrchovina hills, Volica (367), 9.ix.1979, SW, 1M, J. Ježek leg. \& det., slide Inv. No. 20650, NMPC, veg.: Salix, Urtica; Nízke Tatry Mts., Vel’ký Brankov (356), 12.ix.1985, SW, 1F, J. Ježek leg. \& det., slide Inv. No. 20486, NMPC, veg.: Picea, Rubus; Nízke Tatry Mts., Bansko, brook (nr. Liptovská Lúžna) (9), 1181 m a.s.l., 13.ix.1985, SW, 1F, J. Ježek leg. \& det., slide Inv. No. 20569, NMPC, veg.: Picea, Betula, Sambucus, Rubus, Mentha; Nízke Tatry Mts., Liptovská Lúžna (141), 13.ix.1985, SW, 1M, J. Ježek leg. \& det., slide Inv. No. 20286, NMPC, veg.: Salix, Grossularia, Petasites, Mentha, Urtica; Nízke Tatry Mts., Brusno env. Brezno (24), 16.ix.1985, SW, 1F, J. Ježek leg. \& det., slide Inv. No. 20100, NMPC, veg.: Salix, Alnus, Corylus, Urtica, Asarum; Nízke Tatry Mts., Čierny Diel env. Ráztoka (38), 1144 m a.s.l., 18.ix.1985, SW, 1M, J. Ježek leg. \& det., slide Inv. No. 20407, NMPC, veg.: Picea, Salix, Acer, Corylus, Eupatorium, Caltha, Pinguicula; Ondavská vrchovina hills, Makovce (161), 10.ix.1991, SW, 1M, J. Ježek leg. \& det., slide Inv. No. 20450, NMPC, veg.: Alnus, Salix, Rubus, Polygonum, Lappa, Urtica, Poaceae; Ondavská vrchovina hills, Bukovce (25), 11.ix.1991, SW, 1M, J. Ježek leg. \& det., slide Inv. No. 20147, NMPC, veg.: Salix, Alnus, Acer, Rubus, Humulus, Petasites, Lappa, Urtica; Ondavská vrchovina hills, Nová Polianka env. Svidník (207), 12.ix.1991, SW, 1M, J. Ježek leg. \& det., slide Inv. No. 20382, NMPC, veg.: Salix, Alnus, Corylus, Rubus, Typha, Lappa, Urtica, Daucaceae; Laborecká vrchovina hills, Svidnícke sedlo saddleback env. Vyšný Komárnik, brook (312), 13.ix.1991, SW, 1M, J. Ježek leg. \& det., slide Inv. No. 20156, NMPC, veg.: Corylus, Acer, Pirus, Carpinus, Salix, Rubus, Urtica; Ondavská vrchovina hills, Nižná Pisaná, brook (200), 14.ix.1991, SW, 1M, J. Ježek leg. \& det., slide Inv. No. 20209, NMPC, veg.: Alnus, Salix, Carpinus, Geranium, Artemisia, Urtica, Pteropsida, Poaceae; Ondavská vrchovina hills, Bardejovské Kúpele (12), 15.ix.1991, SW, 1F, J. Ježek leg. \& det., slide Inv. No. 20461, NMPC, veg.: Tilia, Salix, Corylus, Frangula, Rubus, Daucaceae, Lamiaceae; Ondavská vrchovina hills, Sveržov (311), 16.ix.1991, SW, 1M, J. Ježek leg. \& det., slide Inv. No. 20219, NMPC, veg.: Salix, Alnus, Polygonum, Petasites, Poaceae; Čergov Mts., Hromacký vrch env. Livov (88), 830 m a.s.l., 26.ix.1992, SW, 1F, J. Ježek leg. \& det., slide Inv. No. 20580, NMPC, veg.: Fagus, Picea, Pinus, Abies, Betula, Mentha, Poaceae; Turčianska kotlina basin, Martin (172), 7.v.1996, 1F, V. Straka leg., J. Ježek det., slide Inv. No. 20664, NMPC; Muránska planina Mts., Muránska planina plain (191), 22.vii.1999, SW, 1M, P. Chvojka leg., J. Ježek det., slide Inv. No. 20300, NMPC; Strážovské vrchy Mts., Diviacka Nová Ves (46), 20.v.2013, 1M, HC, J. Oboňa leg., J. Ježek det., slide Inv. No. 20081, NMPC.

Comments: A very common Holarctic species.

\section{Tinearia Schellenberg, 1803}

\section{Tinearia alternata (Say, 1824)}

Published records: Nitra (195) (Thalhammer 1918); Ružomberok (261) (Szabó 1965a,b); Piešt’any (225) (Ježek 1977); Bratislava (19), Brehy (20), Levice (139), Malacky (167), Michal na Ostrove (179), Nitra (195), Píla (226), Soblahov (273), Trenčín (330), Velčice (351), Žarnovica (385) (Halgoš 1973); Bratislava (19), Brehy (20), Jasná (100), Levice (139), Malacky (167), Michal na Ostrave (177), Nitra (195), Píla (226), Ružomberok (261), Soblahov (273), Trenčín (330), Velčice (351), Žarnovica (385) (Halgoš 1984); Hiadel'ská dolina valley 
(74), Mlynica (183), Štôla (292) (Ježek 1993); Nad Šenkárkou (193) (Straka \& Majzlan 2006); Čierna Lehota (37), Žitná - Radiša (394) (Straka \& Majzlan 2007); Jamy (98), Smrekovec (272) (Straka \& Majzlan 2009); Š́r (309) (Straka \& Majzlan 2010a); Čičarovce (35) (larvae, Mišíková Elexová 2010); Šišulákov mlyn mill (266) (Straka \& Majzlan 2013).

Material examined: Východoslovenská rovina plain, Streda nad Bodrogom (296), 20.v.1977, SW, 1M, J. Burda leg., J. Ježek det., slide Inv. No. 20460, NMPC; Východoslovenská pahorkatina hills, Michal'any (180), 23.viii.1983, SW, 1M, J. Ježek leg. \& det., slide Inv. No. 20236, NMPC, veg.: Salix, Populus, Urtica, Heracleum, Plantago, Ranunculus, Lychnis; Bukovské vrchy Mts., Ubl'ianka (339), 16.vii.1990, SW, 1M, P. Chvojka leg., J. Ježek det., slide Inv. No. 20592, NMPC; Spišsko-šarišské medzihorie Imts., Stará Luubovňa (284), 28.ix.1992, SW, 1M, J. Ježek leg. \& det., slide Inv. No. 20392, NMPC, veg.: Salix, Alnus, Urtica, Lappa; Vel'ká Fatra Mts., Lubochnianska dolina valley (151), 6.xi.2006, reared, 1M, J. Lukáš leg., J. Ježek det., slide Inv. No. 20623, NMPC; Hornonitrianska kotlina basin, Vrbany env. Diviacka Nová Ves (371), 17.v.2012, SW, 1F, J. Oboňa leg., J. Ježek det., slide Inv. No. 20050, NMPC; Strážovské vrchy Mts., Diviacka Nová Ves (46), 22.v., 3.vi. and 24.xi.2012, 3F, all HC, J. Oboňa leg., J. Ježek det., slides Inv. No. 20023, 20032, 20068, NMPC. Comments: A cosmopolitan species, generally very common.

\section{Tinearia lativentris (Berdén, 1952)}

Published record: Mengusovce (178) (Ježek 1993).

Material examined: Východoslovenská rovina plain, Vel'ký Horeš (358), 29.v.1979, SW, 1F, J. Ježek leg. \& det., slide Inv. No. 20542, NMPC, veg.: Salix, Juglans, Iris, Carex, Dactylis, Alisma; Spišská Magura Mts., Osturňa (220), 6.x.1991, SW, 1M, P. Chvojka leg., J. Ježek det., slide Inv. No. 20397, NMPC.

Comments: A Holarctic species, in Slovakia not common.

\section{Ypsydocha Ježek, 1984}

\section{Ypsydocha setigera (Tonnoir, 1922)}

Published records: Ludrová (153), Mlynica (183) (Ježek 1993).

Material examined: Nízke Tatry Mts., Vel'ký Brankov (356), 12.ix.1985, SW, 1F, J. Ježek leg. \& det., slide Inv. No. 20490, NMPC, veg.: Picea, Rubus, Musci; Nízke Tatry Mts., Bansko brook (nr. Liptovská Lúžna) (9), 1181 m a.s.l., 13.ix.1985, SW, 1F, J. Ježek leg. \& det., slide Inv. No. 20563, NMPC, veg.: Picea, Betula, Sambucus, Rubus, Mentha; Nízke Tatry Mts., Liptovská Lúžna, brook (141), 13.ix.1985, SW, 1M, J. Ježek leg. \& det., slide Inv. No. 20323, NMPC, veg.: Picea, Sphagnum, Caltha, Juncus, Mentha, Poaceae; Nízke Tatry Mts., Brusno env. Brezno (24), 16.ix.1985, SW, 1M, J. Ježek leg. \& det., slide Inv. No. 20097, NMPC, veg.: Salix, Alnus, Corylus, Urtica, Asarum; Nízke Tatry Mts., Čierny Diel env. Ráztoka (38), 1144 m a.s.l., 18.ix.1985, SW, 1M, J. Ježek leg. \& det., slide Inv. No. 20409, NMPC, veg.: Picea, Salix, Acer, Corylus, Eupatorium, Caltha, Pinguicula; Laborecká vrchovina hills, Krásny Brod env. Medzilaborce, brook, well (128), 10.ix.1991, SW, 1M, J. Ježek leg. \& det., slide Inv. No. 20121, NMPC, veg.: Alnus, Salix, Corylus, Rubus, Caltha, Mentha, Carex, Dipsacus, Musci; Čergov Mts., Hromacký vrch env. Livov (88), 830 m a.s.l., 26.ix.1992, SW, 1F, J. Ježek leg. \& det., slide Inv. No. 20581, NMPC, veg.: Fagus, Picea, Pinus, Abies, Betula, Mentha, Poaceae.

Comments: The Holarctic species. The larvae are saprobiontic and occur often in manure.

\section{PERICOMINI}

\section{Berdeniella Vaillant, 1976}

\section{Berdeniella gardinii Salamanna \& Raggio, 1984}

Published record: Štôla (292) (Ježek 2009b).

Comments: A very rare Europaen species, the assessment of the current conservation status: NS.

\section{Berdeniella helvetica (Sara, 1957)}

Published records: upper Váh river basin (345) (larvae, Bulánková 2003); Javorov potok brook (102) (larvae, Bulánkova et al. 2012).

Comments: Abnormally rare Europaen species, probably regionally eradicated or extinct, from Slovakia known only from larvae so far, the assessment of the current conservation status in accordance with suggests of the Czech Republic (Ježek 2005): CR. 


\section{Berdeniella illiesi Wagner, 1973}

Published records: Gader (64) (larvae, Bulánková 1991); Turiec (336) (larvae, Bulánková \& Degma 1996); Hučava (93) (larvae, Bulánková 1999, larvae, Bitušík et al. 2000); Hron river basin (89), Latorica river basin (137), Slaná river basin (269), upper Váh river basin (345) (larvae, Bulánková 2003); Nižne Temnosmrečinske pleso mountain lake (202), Vyšne Temnosmrečinske pleso mountain lake (378) (larvae, Bulánková \& Zat’ovičová 2006); Hluboký potok brook (76), Stužická rieka stream (302) (larvae, Novikmec 2007); Čierna Lehota (37), Čierny Hron (40), Liptovská Teplička (142), Liptovské Revúce (145), Liptovský Hrádok (146), Mengusovce (178), Moštenica (188), Nová Lehota (205), Stará Voda (286), Sučany (304), Utekáč (346), Val'kovňa (349), Vel'ký Dvor (357), Vernár (360), Vyšná Maša (375), Železná studnička well (391) (larvae, Mišíková Elexová 2010); Javorov potok brook (102) (larvae, Bulankova et al. 2012).

Comments: Not quite common Europaen species, from Slovakia known only from larvae, traps for a monitoring of the occurence of adults were not installed, unfortunatelly, on the mentioned localities so far.

\section{Berdeniella kocii Ježek, 2006}

Published record: Kyslinky (136) (Ježek 2009a).

Material examined: Vihorlatské vrchy Mts., Zemplínske Hámre, brook (392), 6.ix.1979, SW, 1M, J. Ježek leg. \& det., slide Inv. No. 20337, NMPC, veg.: Salix, Alnus, Urtica; Belianske Tatry Mts., Tristárska dolina valley env. Ždiar (333), 21.vi.1982, SW, 1M, J. Ježek leg. \& det., slide Inv. No. 20424, NMPC, veg.: Picea; Popradská kotlina basin, Tatranská Lesná (318), 23.viii.1983, SW, 1M, J. Ježek leg. \& det., slide Inv. No. 20547, NMPC, veg.: Alnus, Scirpus, Filipendula, Urtica; Vysoké Tatry Mts., Vel'ká Svišt'ovka (354), 9.vii.1987, SW, 1M, J. Ježek leg. \& det., slide Inv. No. 20520, NMPC, veg.: Pinus mugo; Revúcka vrchovina Mts., Rejdová (250), 18.vii.1989, SW, 1M, J. Ježek leg. \& det., slide Inv. No. 20454, NMPC, veg.: Picea, Fagus, Salix, Sambucus, Caltha, Scirpus, Daucaceae.

Comments: A rare montaneous species, know only from Czech Republic and Slovakia; the assessment of the current conservation status NS.

\section{Berdeniella manicata (Tonnoir, 1920)}

Published records: Valča (348) (larvae, Bulánková, 1991); Malá Homôlka (162) (larvae, Bulánková \& Halgoš 1995, larvae, Krno et al. 1995); Turiec (336) (larvae, Bulánková \& Degma 1996); Hučava stream (93), Mičová stream (181) (larvae, Bulánková 1999, larvae, Bitušík et al. 2000); Hron river basin (89), Slaná river basin (269), upper Váh river basin (345) (larvae, Bulánková 2003); Hluboký potok brook (76) (larvae, Novikmec 2007); Bretka (21) (larvae, Mišíková Elexová 2010).

Material examined: Košická kotlina basin, Ruská Nová Ves (260), 23.v.1979, SW, 1M, J. Ježek leg. \& det., slide Inv. No. 20138, NMPC, veg.: Populus, Salix, Fraxinus, Urtica, Ranunculus; Slanské vrchy Mts., Juskova Vol'a env., brook (104), 25.v.1979, SW, 1M, J. Ježek leg. \& det., slide Inv. No. 20196, NMPC, veg.: Fagus, Corylus, Carpinus, Rosa, Ranunculus; Popradská kotlina basin, Tatranská Kotlina (317), 18.vi.1982, SW, 1M, J. Ježek leg. \& det., slide Inv. No. 19970, NMPC, veg.: Picea, Petasites, Musci; Nízke Tatry Mts., Pohronský Bukovec (235), 18.ix.1985, SW, 1M, J. Ježek leg. \& det., slide Inv. No. 20010, NMPC, veg.: Alnus, Caltha, Filipendula; Vysoké Tatry Mts., Malá Studená dolina valley (165), 10.vii.1987, SW, 1M, J. Ježek leg. \& det., slide Inv. No. 20154, NMPC, veg.: Pinus mugo, Betula, Petasites, Luzula, Geranium; Popradská kotlina basin, Červený potok brook - Nová Lesná (34), 22.vii.1990, SW, 1M, P. Chvojka leg., J. Ježek det., slide Inv. No. 20414, NMPC; Spišsko-šarišské medzihorie Imts., Sabinov (262), 24.ix.1992, SW, 1F, J. Ježek leg. \& det., slide Inv. No. 20250, NMPC, veg.: Salix, Corylus, Mentha, Impatiens; Podbeskydská vrchovina Mts., Biela Orava river nr. Lokca (15), 29.vi.1992, SW, 1M, P. Chvojka leg., J. Ježek det., slide Inv. No. 20576, NMPC; Kremnické vrchy Mts., Železná Breznica env., brook (390), 29.iv.2012, 30.iv.2013, both SW, 2M, J. Oboňa leg., J. Ježek det., slides Inv. No. 20047, 20059, NMPC; Kremnické vrchy Mts., Kremnica (129), 17.v.2013, SW, 1M, M. Svitok \& J. Oboňa leg., J. Ježek det., slide Inv. No. 20054, NMPC.

Comments: Relative common European species. Until now, known from Slovakia only as larvae, the mentioned examined material represents first adult records of this species in Slovakia.

\section{Berdeniella matthesi (Jung, 1954)}

Published record: Dolina Bielych ples valley (49) (Ježek 2006a).

Material examined: Belianske Tatry Mts., Tristárska dolina valley env. Ždiar (333), 21.vi.1982, SW, 1M, J. Ježek leg. \& det., slide Inv. No. 20423, NMPC, veg.: Picea; Vysoké Tatry Mts., Holica (77), 1630 m a.s.l., 26.vi.1982, SW, 1M, J. Ježek leg. \& det., slide Inv. No. 20132, NMPC, veg.: Picea, Petasites, Musci; Vysoké Tatry Mts., Suchý vrch env. Javorina (306), 26.vi.1982, SW, 1M, J. Ježek leg. \& det., slide Inv. No. 20016, NMPC, veg.: Picea, Rubus, Petasites, Caltha, Trollius; Podtatranská kotlina basin, Kežmarská Biela Voda 
stream (110), 9.vii.1987, SW, 1M, J. Ježek leg. \& det., slide Inv. No. 20334, NMPC, veg.: Sorbus, Pinus mugo, Rubus, Caltha, Pteropsida.

Comments: A locally common species in Europe, known from hilly areas or mountains in shaded swamps mainly with flocculated iron.

\section{Berdeniella stavniensis (Krek, 1969)}

Published record: Dolný Jelenec (52) (Ježek 2006a).

Material examined: Popradská kotlina basin, Tatranská Kotlina env., Belianska jaskyňa cave, brook (316), 18.vi.1982, SW, 1M, J. Ježek leg. \& det., slide Inv. No. 19972, NMPC, veg.: Picea, Petasites, Musci; Vysoké Tatry Mts., Suchý vrch env. Javorina (306), 26.vi.1982, SW, 1M, J. Ježek leg. \& det., slide Inv. No. 20014, NMPC, veg.: Picea, Rubus, Petasites, Caltha, Trollius; Liptovská kotlina basin, Pavčina Lehota nr. Liptovský Mikuláš (223), 10.vi.1987, SW, 1M, J. Ježek leg. \& det., slide Inv. No. 19944, NMPC, veg.: Salix, Rubus, Caltha, Alisma, Filipendula, Geum, Urtica.

Comments: European species, apparently not quite common.

\section{Berdeniella unispinosa (Tonnoir, 1919)}

Published records: Mlynica (1823), Zbojská (388) (Szabó 1960); Štrbské Pleso (295) (Szabó 1965a); Štrbské pleso mountain lake (294), Zbojská (388) (Szabó 1965b); Štrbské pleso mountain lake (294), Zbojská (388) (Halgoš 1984); Megova dolina 1 valley (176) (larvae, Bulánková \& Halgoš 1995); Turiec (336) (larvae, Bulánková \& Degma 1996); Hučava stream (93), Mičová stream (181) (larvae, Bulánková 1999); Hučava stream (93), Mičová stream (181) (larvae, Bitušík et al. 2000); Hron river basin (89), Latorica river basin (137) (larvae, Bulánková 2003); Hluboký potok brook (76) (larvae, Novikmec 2007); Jamy (98) (Straka \& Majzlan 2009); Revúčka (254), Vyšná Maša (375) (larvae, Mišíková Elexová 2010).

Material examined: Popradská kotlina basin, Tatranská Kotlina env. Belianska jaskyňa cave, brook (317), 18.vi.1982, SW, 1M, J. Ježek leg. \& det., slide Inv. No. 19971, NMPC, veg.: Picea, Petasites, Musci; Liptovská kotlina basin, Tri Studničky env. Pavučia dolina valley (331), 16.viii.1983, SW, 1M, J. Ježek leg. \& det., slide Inv. No. 20416, NMPC, veg.: Picea, Caltha, Urtica; Podtatranská brázda furrow, Biela (16), 6.vii.1989, SW, 1M, P. Chvojka leg., J. Ježek det., slide Inv. No. 20534, NMPC; Revúcka vrchovina Mts., Rejdová (250), 18.vii.1989, SW, 1M, J. Ježek leg. \& det., slide Inv. No. 20453, NMPC, veg.: Picea, Fagus, Salix, Sambucus, Caltha, Scirpus, Daucaceae; Stolické vrchy Mts., Slanská dolina valley env. Rejdová (271), 18.vii.1989, SW, 1M, J. Ježek leg. \& det., slide Inv. No. 20603, NMPC, veg.: Picea, Caltha, Mentha, Impatiens, Pteropsida; Stolické vrchy Mts., Muránska Zdychava (192), 19.vii.1989, SW, 1M, J. Ježek leg. \& det., slide Inv. No. 20142, NMPC, veg.: Alnus, Rubus, Filipendula, Impatiens, Caltha, Pteropsida; Chočské vrchy Mts., Oblazy (210), 3.vii.1992, SW, 1M, P. Chvojka leg., J. Ježek det., slide Inv. No. 20398, NMPC; Chočské vrchy Mts., Valaská Dubová, well (347), 4.vii.1992, SW, 1M, P. Chvojka leg., J. Ježek det., slide Inv. No. 20315, NMPC. Comments: A European species, associated here with forest spring areas, brooks, streams, and wet avalanche scars.

\section{Berdeniella vimmeri Ježek, 1995}

Published record: Malá Lodina (163) (Ježek 2009b).

Material examined: Čierna hora Mts., Čermel’ nr. Košice (31), 19.v.1980, SW, 1M, J. Ježek leg. \& det., slide Inv. No. 20367, NMPC, veg.: Alnus, Salix, Carpinus, Petasites, Urtica; Kremnické vrchy Mts., Železná Breznica env., brook (390), 30.iv.2012, SW, 1M, J. Oboňa leg., J. Ježek det., slide Inv. No. 20043, NMPC.

Comments: A central European species (our knowledge is poor so far); inhabiting banks of forest streams, brooks, and spring areas.

\section{Clytocerus Eaton, 1904 \\ Boreoclytocerus Duckhouse, 1978}

\section{Clytocerus (Boreoclytocerus) dalii (Eaton, 1893)}

Published records: Bardejov (11) (Szabó 1965a,b, Halgoš 1984), Material examined: Čierna hora Mts., Kysak (135), 600 m a.s.l., 19.v.1980, SW, 1M, J. Ježek leg. \& det., slide Inv. No. 20171, NMPC, veg.: Fagus, Carpinus, Juncus, Mentha.

Comments: A European species, occuring rarely in outflows of upland peat-bogs, forest spring areas and inundated meadows. 


\section{Clytocerus (Boreoclytocerus) longicorniculatus Krek, 1987}

Published record: Dulova Ves (59) (Ježek 2009b).

Material examined: Slanské vrchy Mts., Červenica (33), 24.v.1979, SW, 1M, J. Ježek leg. \& det., slide Inv. No. 20278, NMPC, veg.: Alnus, Corylus, Sorbus, Scirpus, Juncus, Equisetum; Slanské vrchy Mts., Tuhrina (335), 24.v.1979, SW, 1M, J. Ježek leg. \& det., slide Inv. No. 20177, NMPC, veg.: Fagus, Carpinus, Alnus, Viola; Východoslovenská pahorkatina hills, Zámutov env. Vranov nad Topl'ou, brook (383), 24.v.1979, SW, 1M, J. Ježek leg. \& det., slide Inv. No. 20107, NMPC, veg.: Fagus, Betula, Mentha, Urtica; Slanské vrchy Mts., Juskova Vol'a env., brook (104), 25.v.1979, SW, 1M, J. Ježek leg. \& det., slide Inv. No. 20192, NMPC, veg.: Fagus, Corylus, Carpinus, Rosa, Ranunculus; Východoslovenská rovina plain, Kapušianske Kl'ačany (107), 29.v.1979, SW, 1M, J. Ježek leg. \& det., slide Inv. No. 20587, NMPC, veg.: Quercus, Fraxinus, Populus, Salix, Iris; Veporské vrchy Mts., Príslop (240), 8.ix.1979, SW, 1M, J. Ježek leg. \& det., slide Inv. No. 20264, NMPC, veg.: Alnus, Juncus, Mentha, Carex; Laborecká vrchovina hills, Stakčín (281), 8.ix.1979, SW, 1M, J. Ježek leg. \& det., slide Inv. No. 20556, NMPC, veg.: Equisetum, Mentha, Juncus, Scirpus, Carex; Čierna hora Mts., Kavečany, brook (109), 19.v.1980, SW, 1M, J. Ježek leg. \& det., slide Inv. No. 20200, NMPC, veg.: Fagus, Salix, Alnus, Petasites, Urtica; Čierna hora Mts., Margecany, brook (169), 20.v.1980, SW, 1M, J. Ježek leg. \& det., slide Inv. No. 20269, NMPC, veg.: Mentha, Carex, Urtica; Popradská kotlina basin, Tatranská Polianka (320), 21.viii.1983, SW, 1M, J. Ježek leg. \& det., slide Inv. No. 20345, NMPC, veg.: Picea, Alnus, Rubus, Caltha, Lysimachia, Filipendula, Urtica; Starohorské vrchy Mts., Stará Pila (285), 1.vi.1984, SW, 1M, J. Ježek leg. \& det., slide Inv. No. 20305, NMPC, veg.: Rubus, Carex, Juncus, Urtica, Musci; Laborecká vrchovina hills, Krásny Brod env. Medzilaborce, brook, well (128), 10.ix.1991, SW, 1M, J. Ježek leg. \& det., slide Inv. No. 20115, NMPC, veg.: Alnus, Salix, Corylus, Rubus, Caltha, Mentha, Carex, Dipsacus, Musci; Ondavská vrchovina hills, Rakovč́́k (246), 12.ix.1991, SW, 1M, J. Ježek leg. \& det., slide Inv. No. 20293, NMPC, veg.: Alnus, Prunus, Corylus, Sambucus, Salix, Cirsium, Poaceae, Daucaceae.

Comments: Probably not common European species. Known only from Bosnia and Herzegovina, Czech Republic and Slovakia (Ježek et al. 2013). The assessment of the current conservation status: NS.

\section{Clytocerus (Boreoclytocerus) ocellaris (Meigen, 1804)}

Published records: Zbojská (388), Remetské Hámre (251) (Szabó 1965a,b); Remetské Hámre (251), Vihorlat (361), Zbojská (388) (Szabó 1972-73); Nová Baňa (204), Revištské Podhradie (252) (Halgoš 1973); Nová Baňa (204), Remetské Hámre (251), Revištské Podhradie (252), Zbojská (388) (Halgoš 1984); Hron river basin (89), Latorica river basin (137) (larvae, Bulánková 2003); Hrochotská dolina valley (87) (Ježek 2009a).

Material examined: Zemplínske vrchy Mts., Viničky (362), 20.v.1977, SW, 1M, J. Burda leg., J. Ježek det., slide Inv. No. 20288, NMPC; Košická kotlina basin, Solivar env. Prešov (275), 23.v.1979, SW, 1M, J. Ježek leg. \& det., slide Inv. No. 20004, NMPC; Slanské vrchy Mts., Červenica (323), 24.v.1979, SW, 1M, J. Ježek leg. \& det.,slide Inv. No. 20281, NMPC, veg.: Alnus, Corylus, Sorbus, Scirpus, Juncus, Equisetum; Slanské vrchy Mts., Lúčina (152), 24.v.1979, SW, 1M, J. Ježek leg. \& det., slide Inv. No. 20258, NMPC, veg.: Betula, Alnus, Mentha, Ajuga, Scirpus; Slanské vrchy Mts., Tuhrina (335), 24.v.1979, SW, 1M, J. Ježek leg. \& det., slide Inv. No. 20178, NMPC, veg.: Fagus, Carpinus, Alnus, Viola; Východoslovenská pahorkatina hills, Zámutov env. Vranov nad Topl'ou, brook (384), 24.v.1979, SW, 1M, J. Ježek leg. \& det., slide Inv. No. 20106, NMPC, veg.: Fagus, Betula, Mentha, Urtica; Slanské vrchy Mts., Banské (8), 25.v.1979, SW, 1M, J. Ježek leg. \& det., slide Inv. No. 20549, NMPC, veg.: Picea, Rubus, Myosotis; Slanské vrchy Mts., Juskova Vol'a env., brook (104), 25.v.1979, SW, 1M, J. Ježek leg. \& det., slide Inv. No. 20195, NMPC, veg.: Fagus, Corylus, Carpinus, Rosa, Ranunculus; Košická kotlina basin, Herl'any (73), 26.v.1979, SW, 1M, J. Ježek leg. \& det., slide Inv. No. 20129, NMPC, veg.: Rosa, Rubus, Asperula, Urtica; Východoslovenská rovina plain, Vel'ký Horeš (358), 29.v.1979, SW, 1M, J. Ježek leg. \& det., slide Inv. No. 20539, NMPC, veg.: Salix, Juglans, Iris, Carex, Dactylis, Alisma; Veporské vrchy Mts., Príslop (240), 8.ix.1979, SW, 1M, J. Ježek leg. \& det., slide Inv. No. 20263, NMPC, veg.: Alnus, Juncus, Mentha, Carex; Laborecká vrchovina hills, Belá nad Cirochou, brook (14), 6.ix.1979, SW, 1M, J. Ježek leg. \& det., slide Inv. No. 19999, NMPC, veg.: Salix, Alnus, Caltha, Urtica; Laborecká vrchovina hills, Nižná Čabina (197), 9.ix.1979, SW, 1M, J. Ježek leg. \& det., slide Inv. No. 20307, NMPC, veg.: Salix, Alnus, Urtica, Phragmites; Čierna hora Mts., Čermel’ nr.Košice (31), 19.v.1980, SW, 1M, J. Ježek leg. \& det., slide Inv. No. 20366, NMPC, veg.: Alnus, Salix, Carpinus, Petasites, Urtica; Čierna hora Mts., Kysak (135), 600 m a.s.l., 19.v.1980, SW, 1M, J. Ježek leg. \& det., slide Inv. No. 20170, NMPC, veg.: Fagus, Carpinus, Juncus, Mentha; Vysoké Tatry Mts., Biely Váh (17), 16.viii.1983, SW, 1M, J. Ježek leg. \& det., slide Inv. No. 20385, NMPC, veg.: Picea, Rubus, Sphagnum, Scirpus, Juncus, Caltha; Vysoké Tatry Mts, Mengusovce (178), 19.viii.1983, SW, 1M, J. Ježek leg. \& det., slide Inv. No. 19985, NMPC, veg.: Betula, Picea, Caltha, Rumex, Urtica; Popradská kotlina basin, Tatranská Polianka (320), 21.viii.1983, SW, 1M, J. Ježek leg. \& det., slide Inv. No. 20346, NMPC, veg.: Picea, Alnus, Rubus, Caltha, Lysimachia, Filipendula, Urtica; Popradská kotlina basin, Dolný Smokovec (53), 22.viii.1983, SW, 1M, J. Ježek leg. \& det., slide Inv. No. 20206, NMPC, veg.: Alnus, Filipendula, Caltha, Urtica; Východoslovenská pahorkatina hills, Michal'any 
(180), 23.viii.1983, SW, 1M, J. Ježek leg. \& det., slide Inv. No. 20235, NMPC, veg.: Salix, Populus, Urtica, Heracleum, Plantago, Ranunculus, Lychnis; Starohorské vrchy Mts., Stará Pila (285), 1.vi.1984, SW, 1M, J. Ježek leg. \& det., slide Inv. No. 20304, NMPC, veg.: Rubus, Carex, Juncus, Urtica, Musci; Nízke Tatry Mts., Liptovská Lúžna, brook (141), 13.ix.1985, SW, 1M, J. Ježek leg. \& det., slide Inv. No. 20322, NMPC, veg.: Picea, Sphagnum, Caltha, Juncus, Mentha, Poaceae; Liptovská kotlina basin, Pavčina Lehota nr. Liptovský Mikuláš (223), 10.vi.1987, SW, 1M, J. Ježek leg. \& det., slide Inv. No. 19950, NMPC, veg.: Salix, Rubus, Caltha, Alisma, Filipendula, Geum, Urtica; Laborecká vrchovina hills, Krásny Brod env. Medzilaborce, brook, well (128), 10.ix.1991, SW, 1M, J. Ježek leg. \& det., slide Inv. No. 20119, NMPC, veg.: Alnus, Salix, Corylus, Rubus, Caltha, Mentha, Carex, Dipsacus, Musci; Ondavská vrchovina hills, Makovce (161), 10.ix.1991, SW, 1M, J. Ježek leg. \& det., slide Inv. No. 20448, NMPC, veg.: Alnus, Salix, Rubus, Polygonum, Lappa, Urtica, Poaceae; Ondavská vrchovina hills, Rakovčík (246), 12.ix.1991, SW, 1F, J. Ježek leg. \& det., slide Inv. No. 20292, NMPC, veg.: Alnus, Prunus, Corylus, Sambucus, Salix, Cirsium, Poaceae, Daucaceae; Laborecká vrchovina hills, Korejovce (117), 13.ix.1991, SW, 1M, J. Ježek leg. \& det., slide Inv. No. 20159, NMPC, veg.: Salix, Alnus, Petasites, Dipsacus, Impatiens, Musci, Poaceae; Ondavská vrchovina hills, Kružlová (131), 14.ix.1991, SW, 1M, J. Ježek leg. \& det., slide Inv. No. 20357, NMPC, veg.: Alnus, Salix, Pinus, Pirus, Betula, Rubus, Petasites, Equisetum; Nižná Pisaná, brook (200), 14.ix.1991, SW, 1M, J. Ježek leg. \& det., slide Inv. No. 20213, NMPC, veg.: Alnus, Salix, Carpinus, Geranium, Artemisia, Urtica, Pteropsida, Poaceae; Ondavská vrchovina hills, Šarišské Čierne (263), 15.ix.1991, SW, 1M, J. Ježek leg. \& det., slide Inv. No. 20186, NMPC, veg.: Salix, Alnus, Pirus, Frangula, Evonymus, Rubus, Urtica, Daucaceae; Podunajská rovina flat, Šúr (309), 7.viii.2009, MT, 1M, J. Lukáš leg., J. Ježek det., slide Inv. No. 20639, NMPC.

Comments: A very common central and western European species.

\section{Clytocerus (Boreoclytocerus) rivosus (Tonnoir, 1919)}

Published records: Račková dolina valley (244) (Rozkošný 1971, Halgoš 1984); upper Váh river basin (345) (larvae, Bulánková 2003); Š́r (309) (Straka \& Majzlan 2010a).

Material examined: Veporské vrchy Mts., Príslop (240), 8.ix.1979, SW, 1M, J. Ježek leg. \& det., slide Inv. No. 20265, NMPC, veg.: Alnus, Juncus, Mentha, Carex.

Comments: Not common European species, with a small abundance in suitable habitats, the assessment of the current conservation status: CR.

\section{Clytocerus (Boreoclytocerus) splendidus Ježek \& Hájek, 2007}

Materiala examined: Slanské vrchy Mts., Červenica (33), 24.v.1979, SW, 1M, J. Ježek leg. \& det., slide Inv. No. 20279, NMPC, veg.: Alnus, Corylus, Sorbus, Scirpus, Juncus, Equisetum; Východoslovenská rovina plain, Leles (138), 29.v.1979, SW, 1M, J. Ježek leg. \& det., slide Inv. No. 20406, NMPC, veg.: Populus, Salix, Iris; Bukovské vrchy Mts., Topol’a (328), 8.ix.1979, SW, 1M, J. Ježek leg. \& det., slide Inv. No. 20417, NMPC, veg.: Alnus, Corylus, Urtica; Popradská kotlina basin, Lučivná - Sviniarka, well, brook (153), 938 m a.s.l., 13.vii.1987, SW, 1M, J. Ježek leg. \& det., slide Inv. No. 19981, NMPC, veg.: Picea, Alnus, Caltha, Filipendula, Equisetum; Nízke Tatry Mts., Liptovská Lúžna, brook (141), 13.ix.1985, SW, 1M, J. Ježek leg. \& det., slide Inv. No. 20320, NMPC, veg.: Picea, Sphagnum, Caltha, Juncus, Mentha, Poaceae.

Comments: Known only from the Czech Republic and Poland (Ježek \& Hájek 2007, Ježek \& Omelková 2012a, Ježek et al. 2013). All above mentioned Slovak localities see Map 7. New species for Slovakia. The assessment of the current conservation status: NS.

\section{Clytocerus (Boreoclytocerus) tetracorniculatus Wagner, 1977}

Materiala examined: Muránska planina Mts., Muránska planina plain (191 - Map 8), 22.vii.1999, SW, 2M, P. Chvojka leg., J. Ježek det., slides Inv. No. 20301 and 20302 NMPC.

Comments: A very rare species, known only from Germany, Czech Republic and Finland (Ježek \& Omelková 2012). New species for Slovakia. The assessment of the current conservation status: CR. 


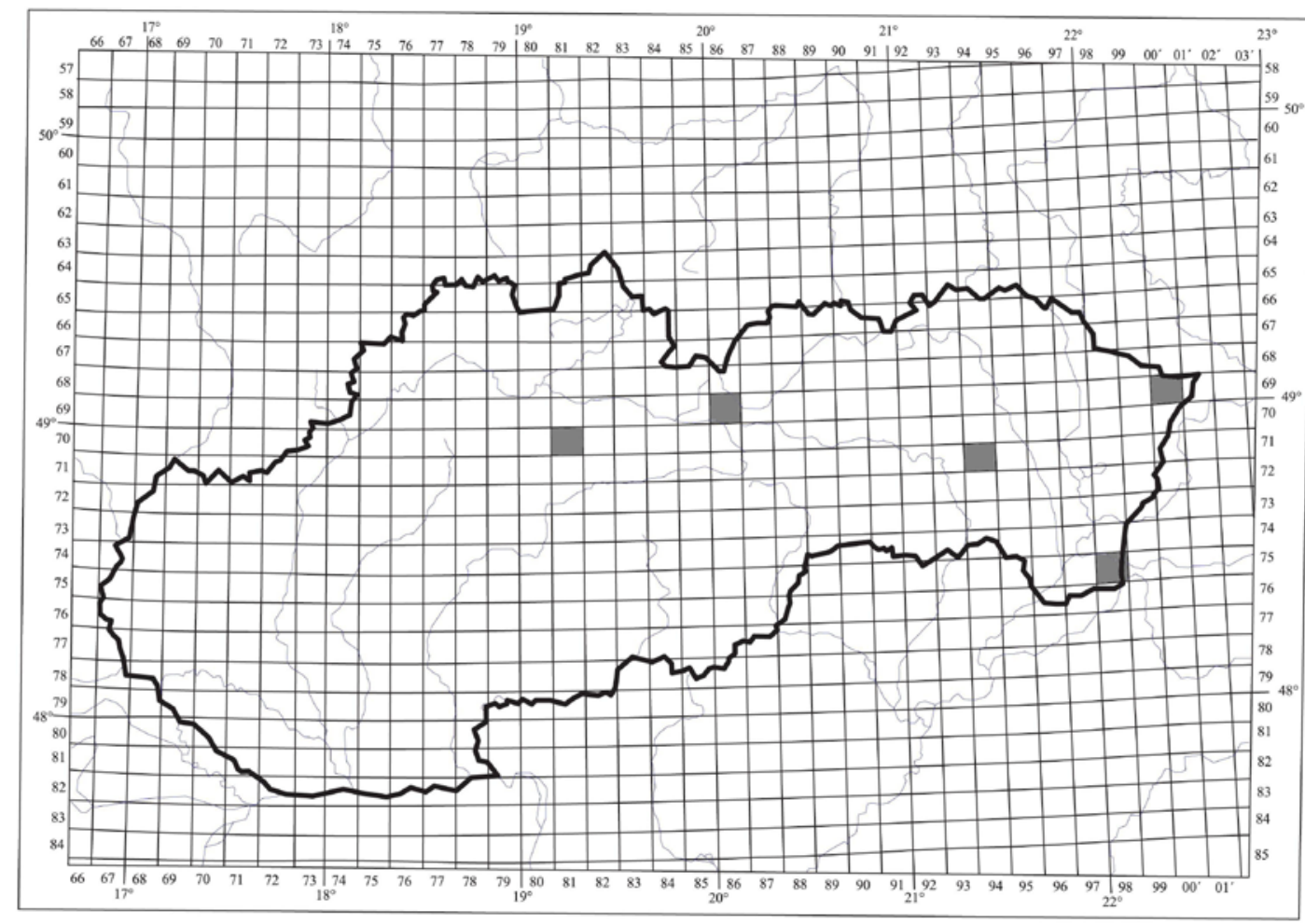

Map 7: Map showing records of Clytocerus (Boreoclytocerus) splendidus Ježek \& Hájek, 2007 from Slovakia (DFS: 6900 - Topol’a, 6986 - Lučivná - Sviniarka, 7081 - Liptovská Lúžna, 7194 - Červenica, 7598 - Leles).

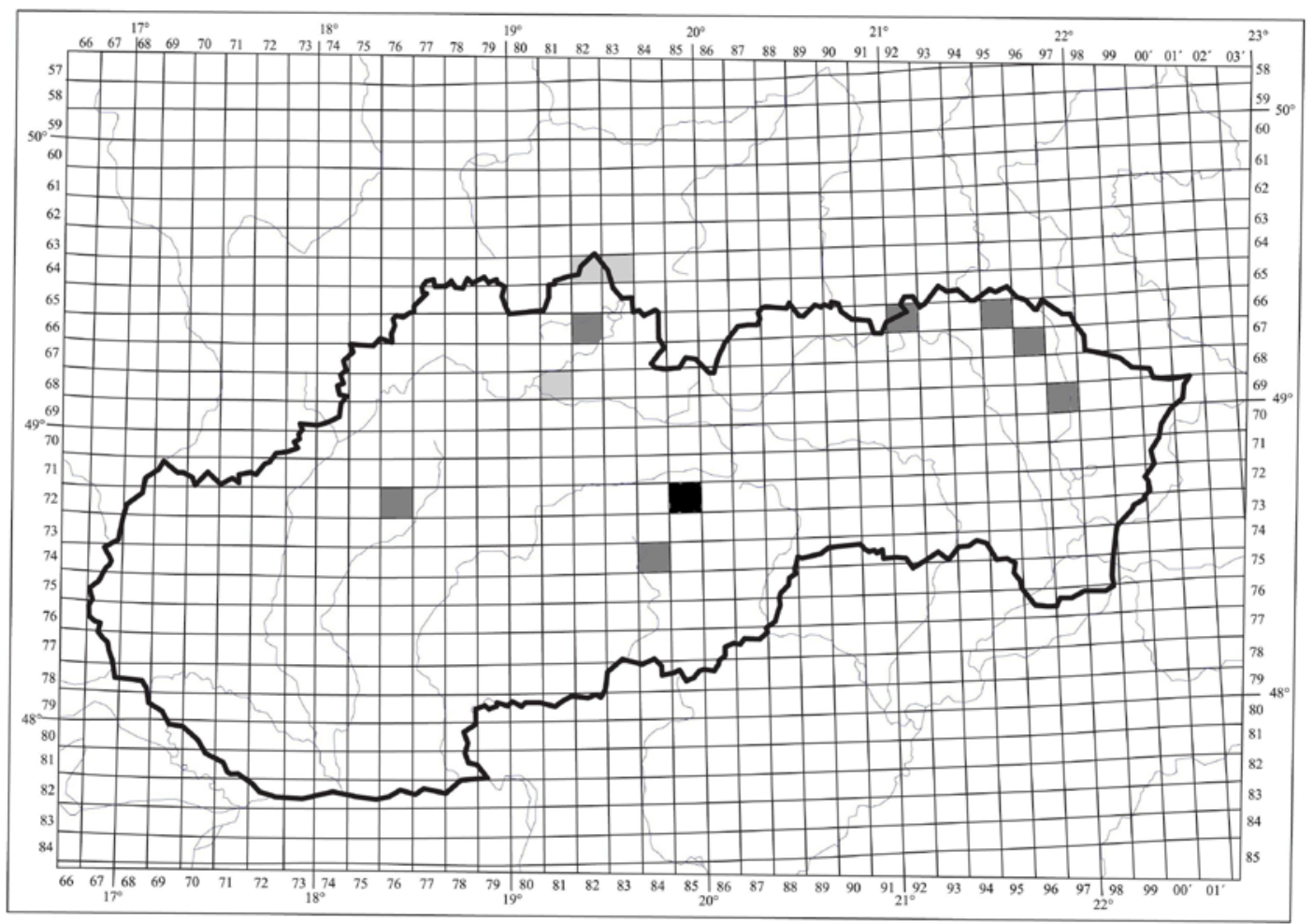

Map 8: Map showing Slovakian records of Clytocerus (Boreoclytocerus) tetracorniculatus Wagner, 1977 black square (DFS: 7285 - Muránska planina plain), Pericoma (Pericoma) exquisita Eaton, 1893 dark gray squares (DFS: 6682 - Biela Orava river, Oravská Jasenica, 6692 - Sveržov, 6695 - Jurkova Vol'a, Korejovce, Kružlová, 6796 - Bukovce, Makovce, 6997 - Hankovce, 7276 - Nitrianske Rudno, 7484 - Príslop) and Pericoma (Pericoma) ljubiniensis Krek, 1969 light gray squares (DFS: 6482 - Oravská Polhora, 6483 - Babia hora Mt., 6881 - Valaská Dubová). 


\section{Parabazarella Vaillant, 1983}

\section{Parabazarella subneglecta (Tonnoir, 1922)}

Published records: Vlkolínec (364) (Szabó 1965a); Hrhov (85) (Rozkošný 1971); Ružomberok (261) (Halgoš 1984); Pavčina Lehota (223) (Ježek 1993); Megova dolina 1, 2 valley $(176,177)$ (larvae, Bulánková \& Halgoš 1995); Turiec (336) (larvae, Bulánková \& Degma 1996); Hučava stream (93), Mičová stream (181) (larvae, Bulánková 1999); Hučava stream (93), Mičová stream (181) (larvae, Bitušík et al. 2000); Dunaj river basin (60), Hron river basin (89), Latorica river basin (137), lower Váh river basin (149), upper Váh river basin (345) (larvae, Bulánková 2003); Nižne Temnosmrečinske pleso mountain lake (202), Vyšne Temnosmrečinske pleso mountain lake (378) (larvae, Bulánková \& Zatovičová 2006); Stupavský potok brook (300) (larvae, Bulánková 2007); Meandre (174) (larvae, Bulánková \& Némethová 2007); Hluboký potok brook (76), Stužická rieka stream (302) (larvae, Novikmec et al. 2007); Jamy (98) (Straka \& Majzlan 2009); Dolný Harmanec (51), Kl'ačno (113), Liptovská Teplička (143), Majdan (160), Mengusovce (178), Stará Voda (286), Utekáč (346) (larvae, Mišíková Elexová 2010).

Material examined: Košická kotlina basin, Ruská Nová Ves (260), 23.v.1979, SW, 1M, J. Ježek leg. \& det., slide Inv. No. 20135, NMPC, veg.: Populus, Salix, Fraxinus, Urtica, Ranunculus; Laborecká vrchovina hills, Hankovce (70), 9.ix.1979, SW, 1M, J. Ježek leg. \& det., slide Inv. No. 20510, NMPC, veg.: Salix, Sambucus, Cornus, Urtica; Čierna hora Mts., Čermel’ nr. Košice (31), 19.v.1980, SW, 1M, J. Ježek leg. \& det., slide Inv. No. 20364, NMPC, veg.: Alnus, Salix, Carpinus, Petasites, Urtica; Popradská kotlina basin, Batizovce env., brook (13), 19.viii.1983, SW, 1M, J. Ježek leg. \& det., slide Inv. No. 19978, NMPC, veg.: Alnus, Grossularia, Caltha; Vysoké Tatry Mts, Mengusovce (178), 19.viii.1983, SW, 1M, J. Ježek leg. \& det., slide Inv. No. 19988, NMPC, veg.: Betula, Picea, Caltha, Rumex, Urtica; Popradská kotlina basin, Dolný Smokovec (53), 22.viii.1983, SW, 1M, J. Ježek leg. \& det., slide Inv. No. 20205, NMPC, veg.: Alnus, Filipendula, Caltha, Urtica; Popradská kotlina basin, Tatranská Lesná (318), 23.viii.1983, SW, 1M, J. Ježek leg. \& det., slide Inv. No. 20545, NMPC, veg.: Alnus, Scirpus, Filipendula, Urtica; Liptovská kotlina basin, Pavčina Lehota nr. Liptovský Mikuláš (223), 10.vi.1987, SW, 1M, J. Ježek leg. \& det., slide Inv. No. 19942, NMPC, veg.: Salix, Rubus, Caltha, Alisma, Filipendula, Geum, Urtica; Laborecká vrchovina hills, Krásny Brod env. Medzilaborce, brook, well (128), 10.ix.1991, SW, 1M, J. Ježek leg. \& det., slide Inv. No. 20114, NMPC, veg.: Alnus, Salix, Corylus, Rubus, Caltha, Mentha, Carex, Dipsacus, Musci; Ondavská vrchovina hills, Makovce (161), 10.ix.1991, SW, 1M, J. Ježek leg. \& det., slide Inv. No. 20447, NMPC, veg.: Alnus, Salix, Rubus, Polygonum, Lappa, Urtica, Poaceae; Ondavská vrchovina hills, Nová Polianka env. Svidník (207), 12.ix.1991, SW, 1M, J. Ježek leg. \& det., slide Inv. No. 20377, NMPC, veg.: Salix, Alnus, Corylus, Rubus, Typha, Lappa, Urtica, Daucaceae; Laborecká vrchovina hills, Korejovce (117), 13.ix.1991, SW, 1M, J. Ježek leg. \& det., slide Inv. No. 20160, NMPC, veg.: Salix, Alnus, Petasites, Dipsacus, Impatiens, Musci, Poaceae; Ondavská vrchovina hills, Jurkova Vol'a (103), 14.ix.1991, SW, 1M, J. Ježek leg. \& det., slide Inv. No. 20504, NMPC, veg.: Salix, Rubus, Petasites, Mentha, Lappa, Artemisia, Urtica, Poaceae; Ondavská vrchovina hills, Sveržov (311), 16.ix.1991, SW, 1M, J. Ježek leg. \& det., slide Inv. No. 20220, NMPC, veg.: Salix, Alnus, Polygonum, Petasites, Poaceae. Comments: A common species known from Europe and Turkey.

\section{Pericoma Walker, 1856 Pachypericoma Vaillant, 1978}

\section{Pericoma (Pachypericoma) blandula Eaton, 1893}

Published records: Rimavská Sobota (256), Vihorlat (361) (Szabó 1965a); Remetské Hámre (251) (Szabó 1965b); Podbanské (232) (Rozkošný 1971); Remetské Hámre (251) (Szabó 1972-73); Brehy (20), Revištské Podhradie (252), Soblahov (273) (Halgoš 1973); Brehy (20), Podbanské (232), Remetské Hámre (251), Revištské Podhradie (252), Soblahov (273) (Halgoš 1984); lower Váh river basin (149), Hron river basin (89), Slaná river basin (269), upper Váh river basin (345) (larvae, Bulánková 2003); Šišulákov mlyn mill (266) (Straka \& Majzlan 2013).

Material examined: Košická kotlina basin, Ruská Nová Ves (260), 23.v.1979, SW, 1M, J. Ježek leg. \& det., slide Inv. No. 20136, NMPC, veg.: Populus, Salix, Fraxinus, Urtica, Ranunculus; Košická kotlina basin, Solivar env. Prešov (275), 23.v.1979, SW, 1M, J. Ježek leg. \& det., slide Inv. No. 20003, NMPC; Košická kotlina basin, Teriakovce env. Prešov (322), 23.v.1979, SW, 1M, J. Ježek leg. \& det., slide Inv. No. 20519, NMPC; Východoslovenská pahorkatina hills, Čaklov (30), 25.v.1979, SW, 1M, J. Ježek leg. \& det., slide Inv. No. 20552, NMPC, veg.: Populus, Juglans, Sambucus, Crataegus, Urtica, Lappa; Východoslovenská pahorkatina hills, Sol' (274), 25.v.1979, SW, 1M, J. Ježek leg. \& det., slide Inv. No. 20434, NMPC, veg.: Alnus, Salix, Sambucus, Urtica; Laborecká vrchovina hills, Dedačov (44), 9.ix.1979, SW, 1M, J. Ježek leg. \& det., slide Inv. No. 20597, NMPC, veg.: Salix, Alnus, Corylus, Asarum, Urtica; Čierna hora Mts., Čermel’ nr. Košice (31), 
19.v.1980, SW, 1M, J. Ježek leg. \& det., slide Inv. No. 20371, NMPC, veg.: Alnus, Salix, Carpinus, Petasites, Urtica; Popradská kotlina basin, Tatranská Lesná (318), 23.viii.1983, SW, 1M, J. Ježek leg. \& det., slide Inv. No. 20546, NMPC, veg.: Alnus, Scirpus, Filipendula, Urtica; Popradská kotlina basin, Červený potok brook Nová Lesná (34), 22.vii.1990, SW, 1M, P. Chvojka leg., J. Ježek det., slide Inv. No. 20413, NMPC; Laborecká vrchovina hills, Krásny Brod env. Medzilaborce, brook, well (128), 10.ix.1991, SW, 1M, J. Ježek leg. \& det., slide Inv. No. 20122, NMPC, veg.: Alnus, Salix, Corylus, Rubus, Caltha, Mentha, Carex, Dipsacus, Musci; Ondavská vrchovina hills, Nová Polianka env. Svidník (207), 12.ix.1991, SW, 1M, J. Ježek leg. \& det., slide Inv. No. 20381, NMPC, veg.: Salix, Alnus, Corylus, Rubus, Typha, Lappa, Urtica, Daucaceae; Laborecká vrchovina hills, Svidnícke sedlo saddleback env. Vyšný Komárnik, brook (312), 13.ix.1991, SW, 1M, J. Ježek leg. \& det., slide Inv. No. 20157, NMPC, veg.: Corylus, Acer, Pirus, Carpinus, Salix, Rubus, Urtica; Muránska planina Mts., Muránska planina plain (191), 22.vii.1999, SW, 1M, P. Chvojka leg., J. Ježek det., slide Inv. No. 20216, NMPC; Strážovské vrchy Mts., Diviacka Nová Ves (46), 20.v.2013, 1M, HC, J. Oboňa leg., J. Ježek det., slide Inv. No. 20084, NMPC; Strážovské vrchy Mts., Trstenec well env. Diviacka Nová Ves (334), 1.vii.2013, 1M, SW, J. Oboňa leg., J. Ježek det., slide Inv. No. 20226, NMPC.

Comments: Species widespread in Europe and recorded also in Transcaucasia, Tunisia, and Morocco.

\section{Pericoma (Pachypericoma) fallax Eaton, 1893}

Published records: Hučava stream (93) (larvae, Bulánková 1999, larvae, Bitušík et al. 2000); Kružlovská Huta (132) (Ježek 2006a).

Material examined: Košická kotlina basin, Kokošovce (115), 23.v.1979, SW, 1M, J. Ježek leg. \& det., slide Inv. No. 20533, NMPC, veg.: Alnus, Populus, Salix, Ranunculus, Geranium, Urtica; Východoslovenská pahorkatina hills, Čaklov (30), 25.v.1979, SW, 1M, J. Ježek leg. \& det., slide Inv. No. 20551, NMPC, veg.: Populus, Juglans, Sambucus, Crataegus, Urtica, Lappa; Slanské vrchy Mts., Juskova Vol'a env., brook (104), 25.v.1979, SW, 1M, J. Ježek leg. \& det., slide Inv. No. 20507, NMPC, veg.: Fagus, Corylus, Carpinus, Rosa, Ranunculus; Východoslovenská pahorkatina hills, Sol' (274), 25.v.1979, SW, 1M, J. Ježek leg. \& det., slide Inv. No. 20433, NMPC, veg.: Alnus, Salix, Sambucus, Urtica; Bukovské vrchy Mts., Kolbasov (116), 6.ix.1979, SW, 1M, J. Ježek leg. \& det., slide Inv. No. 20612, NMPC, veg.: Sambucus, Salix, Alnus, Urtica; Vihorlatské vrchy Mts., Zemplínske Hámre brook (392), 6.ix.1979, SW, 1M, J. Ježek leg. \& det., slide Inv. No. 20336, NMPC, veg.: Salix, Alnus, Urtica; Laborecká vrchovina hills, Dedačov (44), 9.ix.1979, SW, 1M, J. Ježek leg. \& det., slide Inv. No. 20596, NMPC, veg.: Salix, Alnus, Corylus, Asarum, Urtica; Laborecká vrchovina hills, Hankovce (70), 9.ix.1979, SW, 1M, J. Ježek leg. \& det., slide Inv. No. 20513, NMPC, veg.: Salix, Sambucus, Cornus, Urtica; Popradská kotlina basin, Svit, brook (315), 13.vii.1987, SW, 1M, J. Ježek leg. \& det., slide Inv. No. 20396, NMPC, veg.: Salix, Petasites, Urtica; Beskydské predhorie Mts., Ubl’a (338), 16.vii.1990, SW, 1M, P. Chvojka leg., J. Ježek det., slide Inv. No. 20493, NMPC; Bukovské vrchy Mts., Ubl'ianka (339), 16.vii.1990, SW, 1M, P. Chvojka leg., J. Ježek det., slide Inv. No. 20593, NMPC; Oravská vrchovina Mts., Podbiel (233), 3.vi.1991, SW, 1M, P. Chvojka leg., J. Ježek det., slide Inv. No. 20123, NMPC; Laborecká vrchovina hills, Malá Pol'ana (164), 10.ix.1991, SW, 1M, J. Ježek leg. \& det., slide Inv. No. 20349, NMPC, veg.: Fagus, Carpinus, Corylus, Rubus, Melampyrum, Daucaceae; Ondavská vrchovina hills, Makovce (161), 10.ix.1991, SW, 1M, J. Ježek leg. \& det., slide Inv. No. 20452, NMPC, veg.: Alnus, Salix, Rubus, Polygonum, Lappa, Urtica, Poaceae; Ondavská vrchovina hills, Bukovce (25), 11.ix.1991, SW, 1M, J. Ježek leg. \& det., slide Inv. No. 20150, NMPC, veg.: Salix, Alnus, Acer, Rubus, Humulus, Petasites, Lappa, Urtica; Ondavská vrchovina hills, Svidník (313), 12.ix.1991, SW, 1M, J. Ježek leg. \& det., slide Inv. No. 20457, NMPC, veg.: Salix, Alnus, Petasites, Symphytum, Mentha, Impatiens, Poaceae; Laborecká vrchovina hills, Korejovce (117), 13.ix.1991, SW, 1M, J. Ježek leg. \& det., slide Inv. No. 20165, NMPC, veg.: Salix, Alnus, Petasites, Dipsacus, Impatiens, Musci, Poaceae; Ondavská vrchovina hills, Jurkova Vol’a (103), 14.ix.1991, SW, 1M, J. Ježek leg. \& det., slide Inv. No. 20194, NMPC, veg.: Salix, Rubus, Petasites, Mentha, Lappa, Artemisia, Urtica, Poaceae; Ondavská vrchovina hills, Kružlová (131), 14.ix.1991, SW, 1M, J. Ježek leg. \& det., slide Inv. No. 20361, NMPC, veg.: Alnus, Salix, Pinus, Pirus, Betula, Rubus, Petasites, Equisetum; Ondavská vrchovina hills, Nižná Pisaná, brook (200), 14.ix.1991, SW, 1M, J. Ježek leg. \& det., slide Inv. No. 20215, NMPC, veg.: Alnus, Salix, Carpinus, Geranium, Artemisia, Urtica, Pteropsida, Poaceae; Ondavská vrchovina hills, Sveržov (311), 16.ix.1991, SW, 1M, J. Ježek leg. \& det., slide Inv. No. 20222, NMPC, veg.: Salix, Alnus, Polygonum, Petasites, Poaceae; Ondavská vrchovina hills, Gerlachov env. Bardejov (65), 17.ix.1991, SW, 1M, J. Ježek leg. \& det., slide Inv. No. 20376, NMPC, veg.: Alnus, Salix, Rubus, Urtica, Poaceae; Podbeskydská vrchovina Mts., Biela Orava nr. Lokca (15), 29.vi.1992, SW, 1M, P. Chvojka leg., J. Ježek det., slide Inv. No. 20574, NMPC; Chočské vrchy Mts., Kvačianka (133), 2.vii.1992, SW, 1M, P. Chvojka leg., J. Ježek det., slide Inv. No. 20521, NMPC; Ipel’ská pahorkatina hills, Ipel' nr. Šahy (96), 5.vii.1992, 1M, SW, P. Chvojka leg., J. Ježek det., slide Inv. No. 20559, NMPC; Strážovské vrchy Mts., Diviacka Nová Ves (46), 25.v.2012, 20.v.2013, 2M, all HC, J. Oboňa leg., J. Ježek det., slides Inv. No. 20024 and 20085, NMPC.

Comments: A European and western Siberian species. 


\section{Pericoma (Pachypericoma) nielseni Kvifte, 2010}

syn. formosa Nielsen, 1964

Published record: Kružlovská Huta (132) (Ježek 2006a).

Material examined: Ondavská vrchovina hills, Bukovce (25), 11.ix.1991, SW, 1M, J. Ježek leg. \& det., slide Inv. No. 20146, NMPC, veg.: Salix, Alnus, Acer, Rubus, Humulus, Petasites, Lappa, Urtica; Strážovské vrchy Mts., Diviacka Nová Ves (46), 20.v.2013, 1M, HC, J. Oboňa leg., J. Ježek det., slide Inv. No. 20083, NMPC.

Comments: Not common European species, overlooked, published as Pericoma formosa Nielsen, 1964 in the past. The synonymy was explained by Kvifte (2010).

\section{Pericoma s. str.}

\section{Pericoma (Pericoma) calcilega Feuerborn, 1923}

Published records: Soblahov (273) (Halgoš 1973); Oravský Podzámok (219), Soblahov (273) (Halgoš 1984); lower Váh river basin (149), upper Váh river basin (345) (larvae, Bulánková 2003).

Comments: Rather rare European species. The assessment of the current conservation status: EN.

\section{Pericoma (Pericoma) diversa Tonnoir, 1919}

Published records: Bukovinka (28) (larvae, Krno 1992); upper Váh river basin (344) (larvae, Bulánková 2003). Comments: For Slovakia seemingly mysterious species recently. Generally rare European species, from Slovakia known only from larvae so far, traps for a monitoring of the occurence of adults must be installed in future in meanders of the Váh river. The assessment of the current conservation status: NS.

\section{Pericoma (Pericoma) exquisita Eaton, 1893}

Material examined: Veporské vrchy Mts., Príslop (240), 8.ix.1979, SW, 1M, J. Ježek leg. \& det., slide Inv. No. 20267, NMPC, veg.: Alnus, Juncus, Mentha, Carex; Laborecká vrchovina hills, Hankovce (70), 9.ix.1979, SW, 1M, J. Ježek leg. \& det., slide Inv. No. 20511, NMPC, veg.: Salix, Sambucus, Cornus, Urtica; Ondavská vrchovina hills, Makovce (161), 10.ix.1991, SW, 1M, J. Ježek leg. \& det., slide Inv. No. 20449, NMPC, veg.: Alnus, Salix, Rubus, Polygonum, Lappa, Urtica, Poaceae; Ondavská vrchovina hills, Bukovce (25), 11.ix.1991, SW, 1M, J. Ježek leg. \& det., slide Inv. No. 20145, NMPC, veg.: Salix, Alnus, Acer, Rubus, Humulus, Petasites, Lappa, Urtica; Laborecká vrchovina hills, Korejovce (117), 13.ix.1991, SW, 1M, J. Ježek leg. \& det., slide Inv. No. 20162, NMPC, veg.: Salix, Alnus, Petasites, Dipsacus, Impatiens, Musci, Poaceae; Ondavská vrchovina hills, Jurkova Vol'a (103), 14.ix.1991, SW, 1M, J. Ježek leg. \& det., slide Inv. No. 20506, NMPC, veg.: Salix, Rubus, Petasites, Mentha, Lappa, Artemisia, Urtica, Poaceae; Ondavská vrchovina hills, Kružlová (131), 14.ix.1991, SW, 1M, J. Ježek leg. \& det., slide Inv. No. 20356, NMPC, veg.: Alnus, Salix, Pinus, Pirus, Betula, Rubus, Petasites, Equisetum; Ondavská vrchovina hills, Sveržov (311), 16.ix.1991, SW, 1M, J. Ježek leg. \& det., slide Inv. No. 20221, NMPC, veg.: Salix, Alnus, Polygonum, Petasites, Poaceae; Podbeskydská vrchovina Mts., Biela Orava nr. Lokca (15), 29.vi.1992, SW, 1M, P. Chvojka leg., J. Ježek det., slide Inv. No. 20573, NMPC; Oravská kotlina basin, Oravská Jasenica (216), 29.vi.1992, SW, 1M, P. Chvojka leg., J. Ježek det., slide Inv. No. 20614, NMPC; Hornonitrianska kotlina basin, Nitrianske Rudno, dyke drain, southern part of water reservoir (196), $48^{\circ} 48^{\prime} 10.1$ " N, 18²9' 8.6" E, 305 m a.s.1., 28.vii.2012, SW, 2M, J. Oboňa leg., J. Ježek det., slides Inv. No. 20027 and 20028, NMPC.

Comments: Species widespread in Europe, North Africa and Transcaucasia. In Europe known from Albania, Austria, Belgium, Bulgaria, Czech Republic, Crete, Croatia, France, Germany, Great Britain, Greece, Hungary, Ireland, Poland and Slovenia (Ježek 2009, Wagner 2011, Kvifte et al. 2013). Morphological characters of the species see Figs 5, 6: 1-14. All above mentioned localities are included in Map 8. New species for Slovakia. The assessment of the current conservation status: EN. 


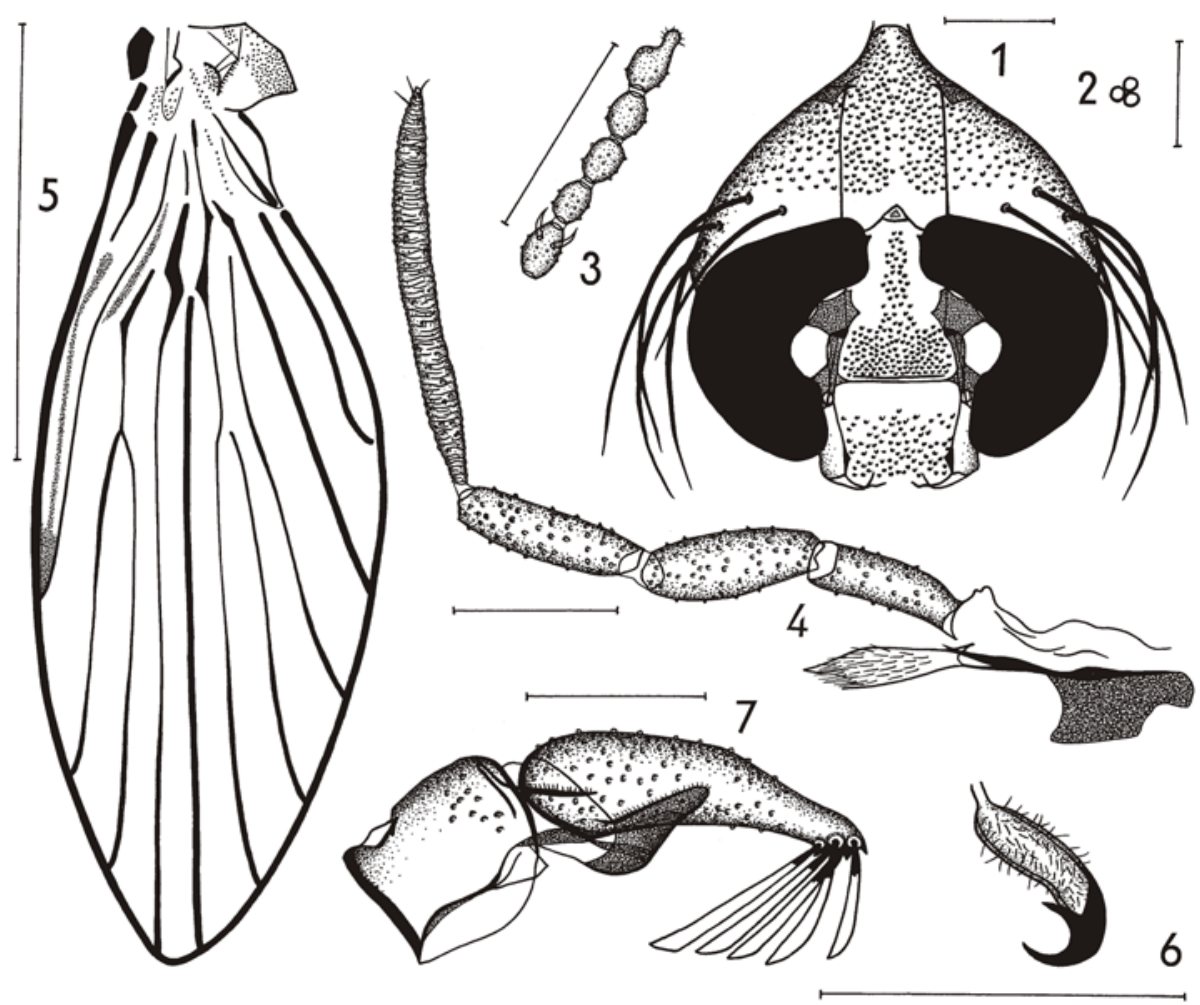

Fig 5: Pericoma (Pericoma) exquisita Eaton, 1893, male. 1 - head; 2 - facets; 3 - terminal antennomeres; 4 maxilla and palpus maxillaris; 5 - wing; 6 - claw of P1; 7 - epandrium and surstyli, lateral view. Scales mostly $0.1 \mathrm{~mm}, 0.05 \mathrm{~mm}$ (6), $1 \mathrm{~mm}$ (5).

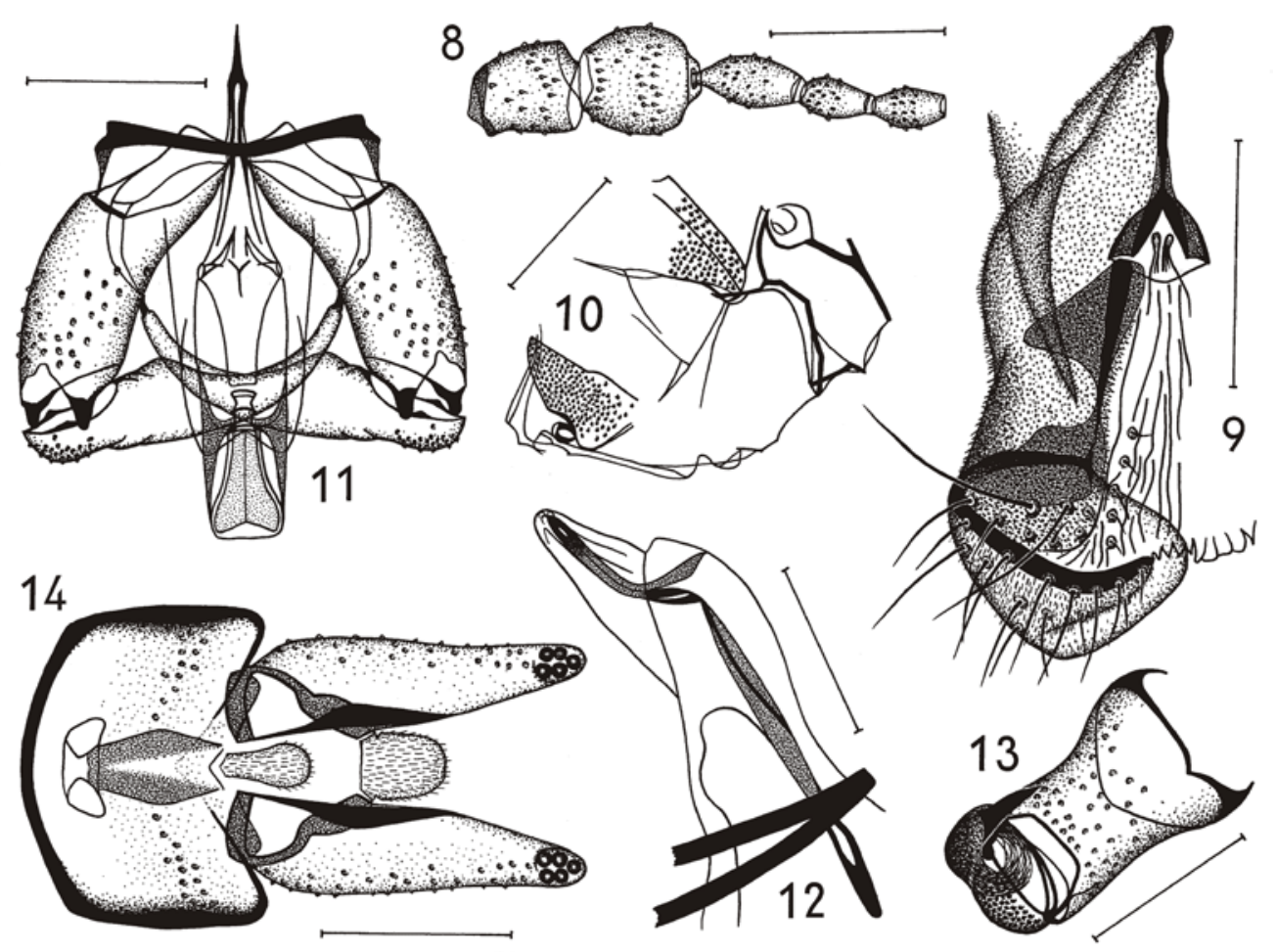

Fig 6: Pericoma (Pericoma) exquisita Eaton, 1893, male. 8 - basal antennomeres; 9 - terminal lobe of labium; 10 - thoracal sclerites; 11 - aedeagal complex and gonopods, dorsall view; 12 - aedeagal complex, lateral view; 13 - gonopod, lateral view; 14 - epandrium and surstyli, dorsal view. Scales mostly $0.1 \mathrm{~mm}, 0.05 \mathrm{~mm}$ (9), 0.2 $\mathrm{mm}(10)$. 


\section{Pericoma (Pericoma) ljubiniensis Krek, 1969}

Materiala examined: Oravské Beskydy Mts., Babia hora Mt. env. Bystré (4), 1300 m a.s.l., 30.vi.1992, SW, 1M, P. Chvojka leg., J. Ježek det., slide Inv. No. 20326, NMPC; Podbeskydská brázda furrow, Oravská Polhora env. Borsučie (217), 30.vi.1992, SW, 1M, P. Chvojka leg., J. Ježek det., slide Inv. No. 20253, NMPC; Chočské vrchy Mts., Valaská Dubová, well (347), 4.vii.1992, SW, 1M, P. Chvojka leg., J. Ježek det., slide Inv. No. 20317, NMPC.

Comments: A scarce species, so far known only from Bosnia and the Czech Republic (Ježek \& Omelková 2012). All above mentioned localities are included in Map 8. New species for Slovakia. The assessment of the current conservation status: CR.

\section{Pericoma (Pericoma) pallida Vaillant, 1978}

Published record: Margecany (169) (Ježek 2009b).

Material examined: Nízke Tatry Mts., Pohronský Bukovec (235), 18.ix.1985, SW, 1M, J. Ježek leg. \& det., slide Inv. No. 20011, NMPC, veg.: Alnus, Caltha, Filipendula; Popradská kotlina basin, Lučivná - Sviniarka (153), 938 m a.s.l., 13.vii.1987, SW, 1M, J. Ježek leg. \& det., slide Inv. No. 19939, NMPC, veg.: Picea, Alnus, Caltha, Filipendula, Equisetum; Laborecká vrchovina hills, Krásny Brod env. Medzilaborce, brook, well (128), 10.ix.1991, SW, 1M, J. Ježek leg. \& det., slide Inv. No. 20120, NMPC, veg.: Alnus, Salix, Corylus, Rubus, Caltha, Mentha, Carex, Dipsacus, Musci; Chočské vrchy Mts., Valaská Dubová, well (347), 6.vii.1994, SW, 1F, P. Lauterer leg., J. Ježek det., slide Inv. No. 20316, NMPC; Strážovské vrchy Mts., env. Diviacka Nová Ves, well - Olavec (212), 290 m a.s.1., 22.vi.2012, SW, 1M, J. Oboňa leg., J. Ježek det., slide Inv. No. 20030, NMPC. Comments: A rare species known only from Spain, Czech Republic and Slovakia. The assessment of the current conservation status: NS.

\section{Pericoma (Pericoma) pingarestica Vaillant, 1978}

Published record: Furmanec river (63) (Ježek 2006a).

Material examined: Strážovské vrchy Mts., Trstenec well env. Diviacka Nová Ves (334), 1.vii.2013, 1M, SW, J. Oboňa leg., J. Ježek det., slide Inv. No. 20224, NMPC.

Comments: A rare European species. The assessment of the current conservation status: NS.

\section{Pericoma (Pericoma) pseudoexquisita Tonnoir, 1940}

Published records: Gidra stream (67) (larvae, Bulánková et al. 2001, Bulánková 2002); lower Váh river basin (149) (larvae, Bulánková 2003); Majdan (160), Mengusovce (178), Host’ovce (82) (larvae, Mišíková Elexová 2010).

Material examined: Strážovské vrchy Mts., Diviacka Nová Ves (46), 20.v.2013, 1M, HC, J. Oboňa leg., J. Ježek det., slide Inv. No. 20086, NMPC.

Comments: A not common European species. Morphological characters of the species see Figs 7, 8: 15-28. Until now, known from Slovakia only as larvae, this is first adult record of this species in Slovakia.

\section{Pericoma (Pericoma) tatrica Szabó, 1960}

Published records: Mlynica (183) (Szabó 1960, 1965a); upper Váh river basin (345) (larvae, Bulánková 2003). Comments: Rare central European species. The assessment of the current conservation status: NS.

\section{Pericoma (Pericoma) tonnoiri Vaillant, 1978}

syn. Pericoma calcilega auct. nec Feuerborn, 1923

Published records: Oravský Podzámok (219) (Tonnoir 1940 as calcilega, Szabó 1965a,b as calcilega, Vaillant 1978 as tonnoiri).

Comments: A rare European species. The assessment of the current conservation status: NS.

\section{Pericoma (Pericoma) trifasciata (Meigen, 1804)}

Published records: Vlkolínec (364) (Szabó 1965a); Ružomberok (261) (Szabó 1972-73, Halgoš 1984); Slaná river basin (269), upper Váh river basin (345) (larvae, Bulánková 2003).

Material examined: Liptovská kotlina basin, Liptovský Hrádok (146), 8.ix.1986, SW, 1M, J. Ježek leg. \& det., slide Inv. No. 19954, NMPC, veg.: Alnus, Cratageus, Salix, Picea, Petasites, Urtica.

Comments: A locally occurring European species. 


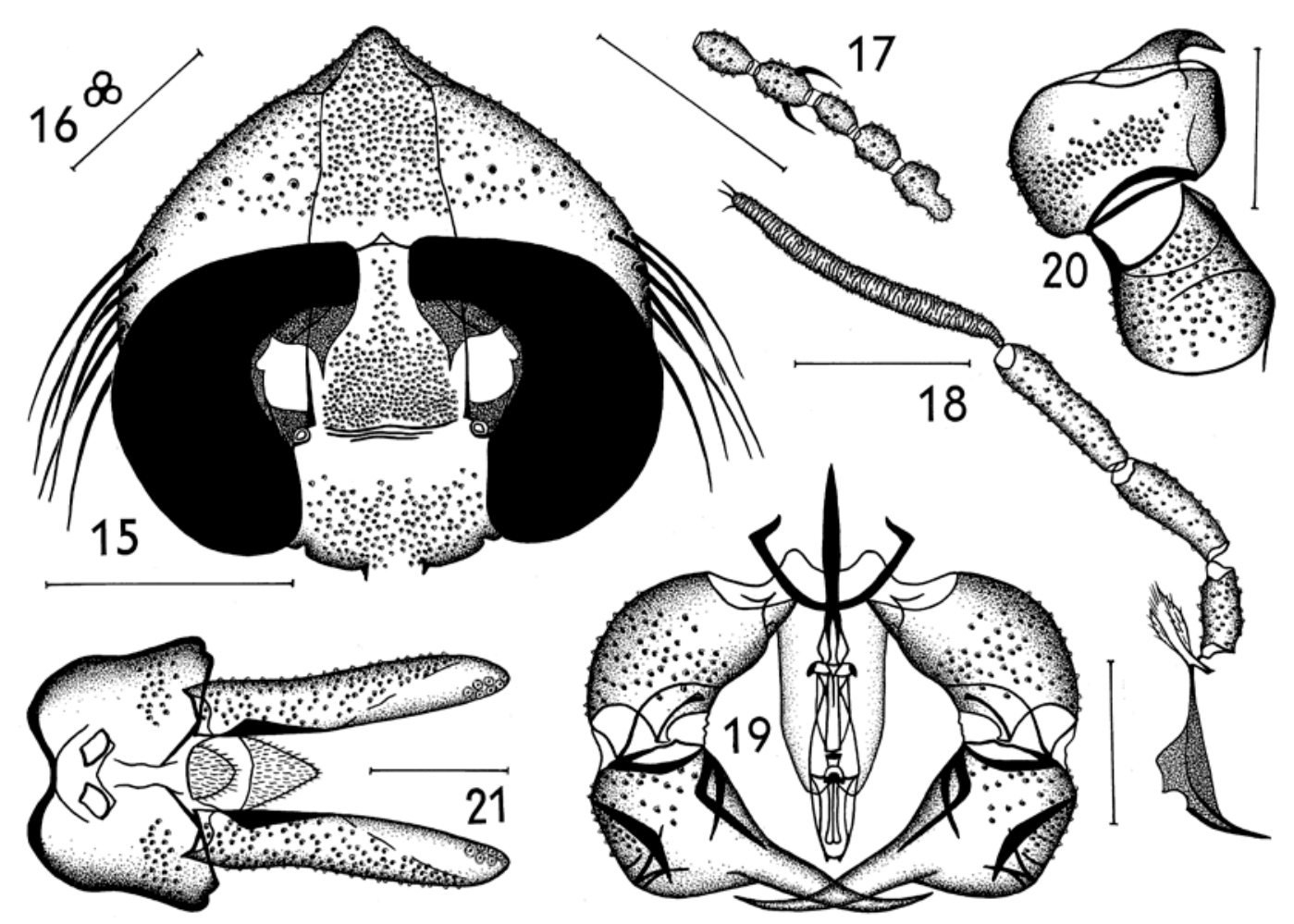

Fig 7: Pericoma (Pericoma) pseudoexquisita Tonnoir, 1940, male. 15 - head; 16 - facets; 17 - terminal antennomeres; 18 - maxilla and palpus maxillaris; 19 - aedeagal complex and gonopods, dorsall view; 20 gonopod, lateral view; 21 - epandrium and surstyli, dorsal view. Scales mostly $0.1 \mathrm{~mm}, 0.2 \mathrm{~mm}$ (15).

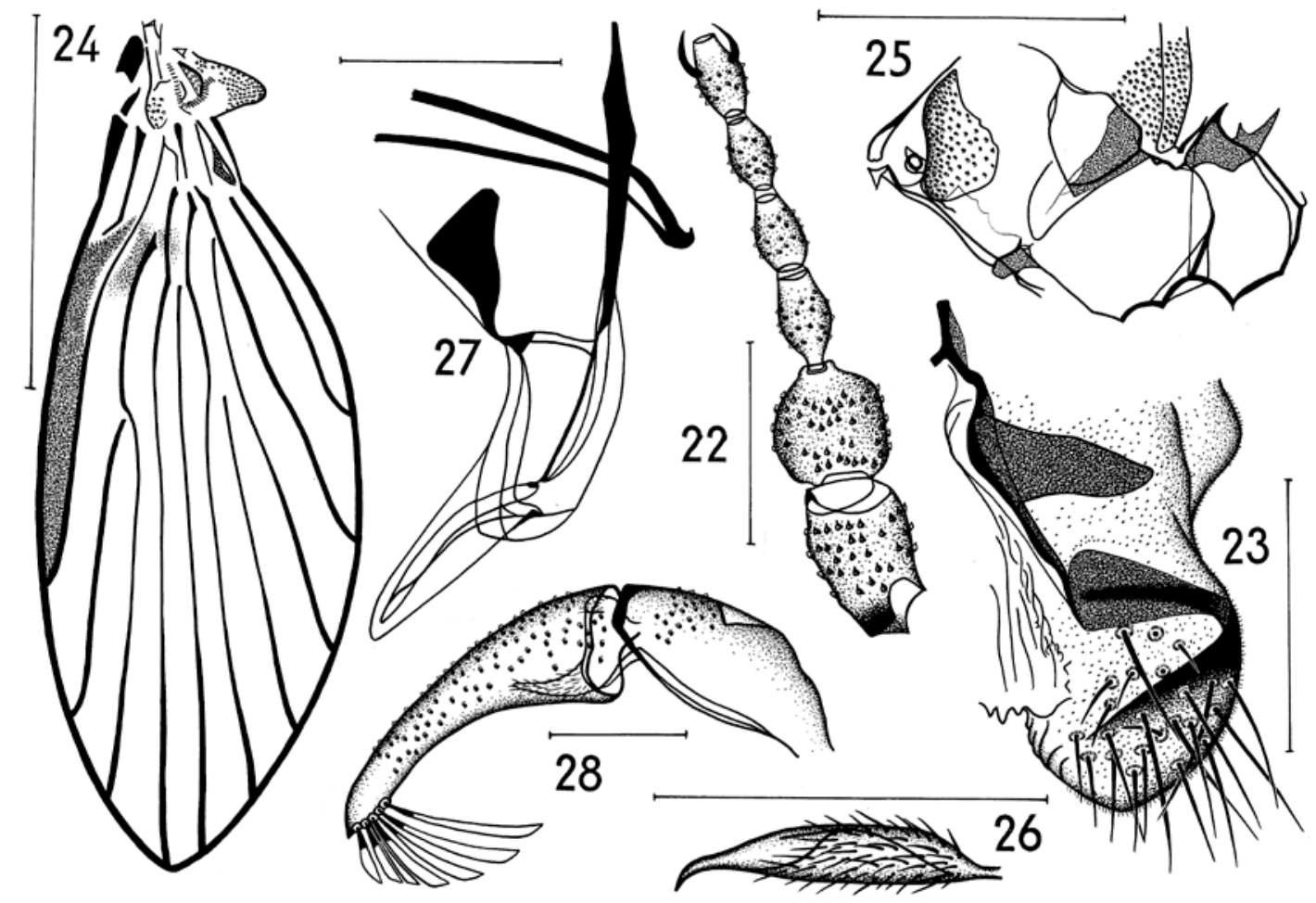

Fig 8: Pericoma (Pericoma) pseudoexquisita Tonnoir, 1940, male. 22 - basal antennomeres; 23 - terminal lobe of labium; 24 - wing; 25 - thoracal sclerites; 26 - claw of P1; 27 - aedeagal complex, lateral view; 28 epandrium and surstyli, lateral view. Scales mostly $0.1 \mathrm{~mm}, 1 \mathrm{~mm}$ (24), $0.5 \mathrm{~mm}$ (25), $0.05 \mathrm{~mm}$ (26). 


\section{Pericoma (Pericoma) vestita Vaillant \& Withers, 1993}

Materiala examined: Muránska planina Mts., Hrdzavá (84), 19.vii.1999, SW, 1M, P. Chvojka leg., J. Ježek det., slide Inv. No. 20555, NMPC.

Comments: A rare European species, known only from the Czech Republic, France and Germany (Ježek \& Omelková 2012). The above mentioned locality is included in Map 9. New species for Slovakia. The assessment of the current conservation status: NS.

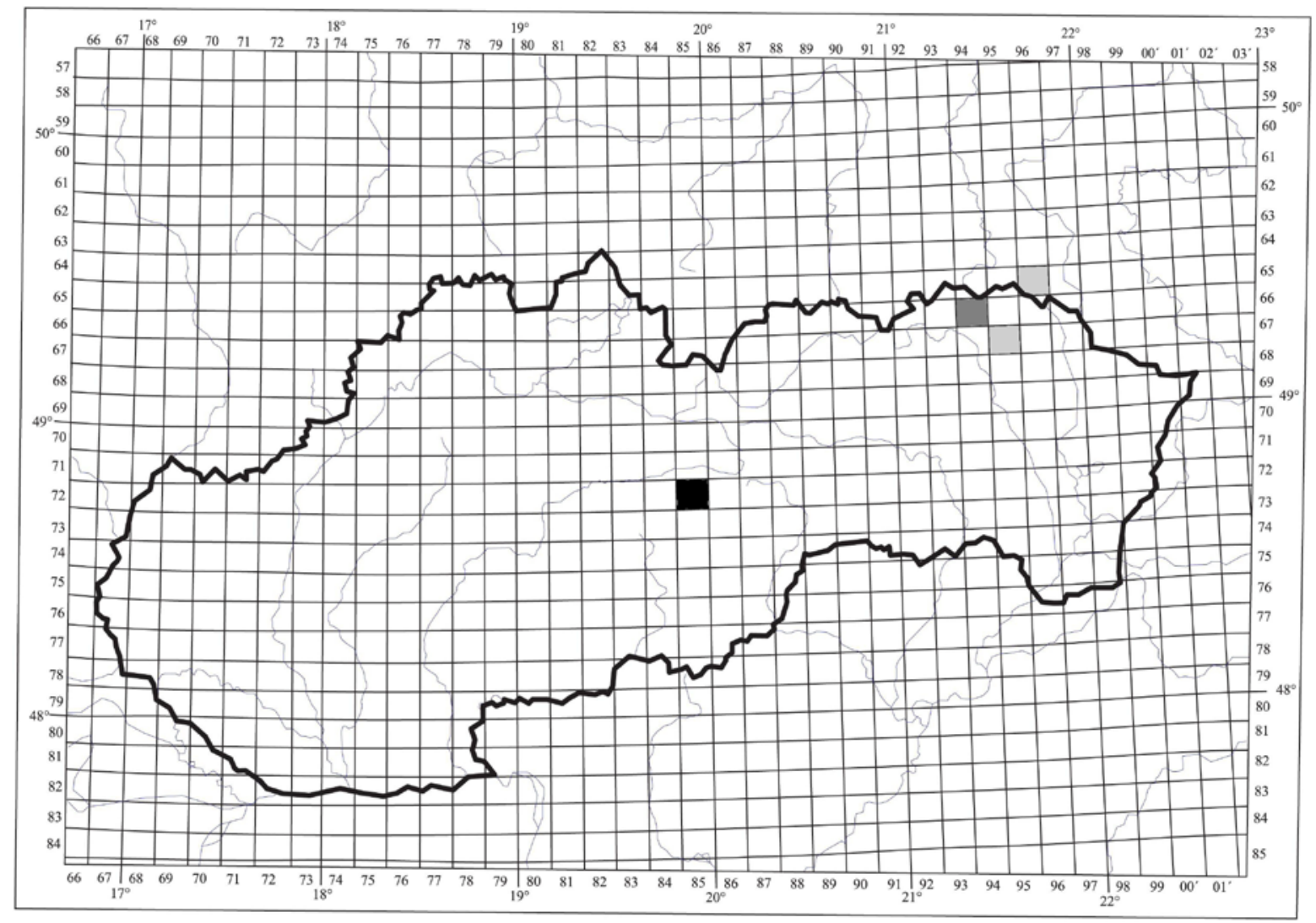

Map 9: Map showing Slovakian records of Pericoma (Pericoma) vestita Vaillant \& Withers, 1993 black square (DFS: 7285 - Hrdzavá), Pneumia compta (Eaton, 1893) dark gray square (DFS: 6694 - Šarišské Čierne) and Pneumia kabelaki Omelková \& Ježek, 2012 light gray squares (DFS: 6596 - Svidnícke sedlo saddleback, 6795 Nová Polianka).

\section{Pneumia Enderlein, 1935}

\section{Pneumia canescens (Meigen, 1804)}

Published records: Bratislava (19) (larvae, Mayer 1938); Il’anovo (94), Liptovské Kl'ačany (1434) (Ježek 1993); upper Váh river basin (345) (larvae, Bulánková 2003).

Material examined: Laborecká vrchovina hills, Krásny Brod env. Medzilaborce, brook, well (128), 10.ix.1991, SW, 1M, J. Ježek leg. \& det., slide Inv. No. 20112, NMPC, veg.: Alnus, Salix, Corylus, Rubus, Caltha, Mentha, Carex, Dipsacus, Musci; Malé Karpaty Mts., Dúbravka (57), 18.viii.2011, SW, 1M, J. Lukáš leg., J. Ježek det., slide Inv. No. 20627, NMPC; Malé Karpaty Mts., Mokrý potok brook (184), 210 - 309 m a.s.l., 25.viii.2011, SW, 2M, J. Lukáš leg., J. Ježek det., slides Inv. No. 20641 and 20649, NMPC; Malé Karpaty Mts., Fialkový potok brook (62), 224 - 247 m a.s.l., 4.ix.2011, SW, 2M, J. Lukáš leg., J. Ježek det., slides Inv. No. 20632 and 20636, NMPC.

Comments: A common species, widely distributed in Europe, western Siberia and Afghanistan. In Slovakia not quite common. 


\section{Pneumia compta (Eaton, 1893)}

Materiala examined: Ondavská vrchovina hills, Šarišské Čierne (263), 15.ix.1991, SW, 3M, J. Ježek leg. \& det., slides Inv. No. 20183, 20184 and 20185, NMPC, veg.: Salix, Alnus, Pirus, Frangula, Evonymus, Rubus, Urtica, Daucaceae.

Comments: A rare species known from Austria, Belgium, Czech Republic, Denmark, Germany, Great Britain, Ireland, Italy, and the Netherlands (Ježek \& Omelková 2012). The above mentioned locality is included in Map 9. New species for Slovakia. The assessment of the current conservation status: NS.

\section{Pneumia crispi (Freeman, 1953)}

Published record: Stužica (301) (Ježek 2006a).

Material examined: Bukovské vrchy Mts., Havešová (71), 12.viii.2003, SW, 1M, J. Ševčík leg., J. Ježek det., slide Inv. No. 12024, NMPC; Laborecká vrchovina hills, Starina env., brook (288), 13.viii.2003, SW, 1M, J. Ševčík leg., J. Ježek det., slide Inv. No. 12029, NMPC, veg. Alnus, Fagus, Carpinus.

Comments: A rare European species. The assessment of the current conservation status: EN.

\section{Pneumia cubitospinosa (Jung, 1954)}

Published record: Stužica (301) (Ježek 2006a).

Comments: A rare European species. The assessment of the current conservation status: EN.

\section{Pneumia fonticola (Szabó, 1960)}

Published records: Košická Belá (123), Zlatá Baňa (395) (Ježek 2003); Hrochotská dolina valley (87) (Ježek 2009a).

Material examined: Bukovské vrchy Mts., Nová Sedlica (208), 9.v.-3.vii. 2002, YPT, 1M, J. Ševčík leg., J. Ježek det., slide Inv. No. 19961, NMPC; Bukovské vrchy Mts., Havešová (71), 12.viii.2003, SW, 1M, J. Ševčík leg., J. Ježek det., slide Inv. No. 12023, NMPC; Laborecká vrchovina hills, Starina env., brook (2878), 13.viii.2003, SW, 1M, J. Ševčík leg., J. Ježek det., slide Inv. No. 12027, NMPC, veg. Alnus, Fagus, Carpinus; Kremnické vrchy Mts., Železná Breznica env., brook (390), 30.iv.2012, 30.iv.2013, SW, 2M, J. Oboňa leg., J. Ježek det., slides Inv. No. 20037 and 20060, NMPC.

Comments: A European species. The assessment of the current conservation status: NS.

\section{Pneumia gracilis gracilis (Eaton, 1893)}

Published records: Fialkový potok brook (62), Mokrý potok brook (184), Vel'kolúcky potok brook (355) (Ježek et al. 2012).

Material examined: Košická kotlina basin, Ruská Nová Ves (260), 23.v.1979, SW, 1M, J. Ježek leg. \& det., slide Inv. No. 20137, NMPC, veg.: Populus, Salix, Fraxinus, Urtica, Ranunculus; Čierna hora Mts., Kavečany brook (109), 19.v.1980, SW, 1M, J. Ježek leg. \& det., slide Inv. No. 20202, NMPC, veg.: Fagus, Salix, Alnus, Petasites, Urtica; Laborecká vrchovina hills, Krásny Brod env. Medzilaborce, brook, well (128), 10.ix.1991, SW, 1M, J. Ježek leg. \& det., slide Inv. No. 20118, NMPC, veg.: Alnus, Salix, Corylus, Rubus, Caltha, Mentha, Carex, Dipsacus, Musci; Kremnické vrchy Mts., Železná Breznica env., brook (390), 29.iv.2012, SW, 1M, J. Oboňa leg., J. Ježek det., slide Inv. No. 20046, NMPC.

Comments: A European species, published from several countries, not too frequent.

\section{Pneumia kabelaki Omelková \& Ježek, 2012}

Materiala examined: Ondavská vrchovina hills, Nová Polianka env. Svidník (207), 12.ix.1991, SW, 2M, J. Ježek leg. \& det., slides Inv. No. 20379 and 20380, NMPC, veg.: Salix, Alnus, Corylus, Rubus, Typha, Lappa, Urtica, Daucaceae; Laborecká vrchovina hills, Svidnícke sedlo saddleback env. Vyšný Komárnik, brook (313), 13.ix.1991, SW, 1M, J. Ježek leg. \& det., slide Inv. No. 20158, NMPC, veg.: Corylus, Acer, Pirus, Carpinus, Salix, Rubus, Urtica.

Comments: Probably a central European species, known only from the Czech Republic (Omelková \& Ježek 2012a). The above mentioned localities are included in Map 9. New species for Slovakia. The assessment of the current conservation status: NS. 


\section{Pneumia mutua (Eaton, 1893)}

Published records: Vyšné Hágy (376) (Szabó 1965a,b); Račková dolina valley (244 - Fig. 9) (Rozkošný 1971); Račková dolina valley (244), Vyšné Hágy (376) (Halgoš 1984); Baláže (6), Bystrá (29), Kropácová (130), Lomnistá dolina valley (148), Piesky (224), Staré Hory (287), Strmý vrštek hill (298), Špania Dolina (280), Tále (316) (Ježek 1993); Hučava stream (93), Mičová stream (181) (larvae, Bulánková 1999, larvae, Bitušík et al. 2000); Hron river basin (89), upper Váh river basin (345) (larvae, Bulánková 2003); Hrončecký grúň slope (90) (Ježek 2009a).

Material examined: Slanské vrchy Mts., Červenica (33), 24.v.1979, SW, 1M, J. Ježek leg. \& det., slide Inv. No. 20276, NMPC, veg.: Alnus, Corylus, Sorbus, Scirpus, Juncus, Equisetum; Slanské vrchy Mts., Tuhrina (335), 24.v.1979, SW, 1M, J. Ježek leg. \& det., slide Inv. No. 20173, NMPC, veg.: Fagus, Carpinus, Alnus, Viola; Košická kotlina basin, Herl'any (3), 26.v.1979, SW, 1M, J. Ježek leg. \& det., slide Inv. No. 20128, NMPC, veg.: Rosa, Rubus, Asperula, Urtica; Košická kotlina basin, Vyšná Kamenica (374), 26.v.1979, SW, 1M, J. Ježek leg. \& det., slide Inv. No. 20255, NMPC, veg.: Fagus, Acer, Impatiens, Asperula, Pteropsida; Košická kotlina basin, Košický Klečenov (124), 26.v.1979, SW, 1M, J. Ježek leg. \& det., slide Inv. No. 20432, NMPC, veg.: Fagus, Asarum, Trifolium, Asperula; Popradská kotlina basin, Tatranská Kotlina (317), 16.vi.1982, SW, 1M, J. Ježek leg. \& det., slide Inv. No. 19990, NMPC, veg.: Picea, Caltha; Vysoké Tatry Mts., Lysá Pol'ana env., Žabí potok brook (158), 24.vi.1982, SW, 1M, J. Ježek leg. \& det., slide Inv. No. 19998, NMPC, veg.: Picea; Vysoké Tatry Mts., Holica (77), 1630 m a.s.l., 26.vi.1982, SW, 1M, J. Ježek leg. \& det., slide Inv. No. 20134, NMPC, veg.: Picea, Petasites, Musci; Vysoké Tatry Mts., Suchý vrch env. Javorina (306), 26.vi.1982, SW, 1M, J. Ježek leg. \& det., slide Inv. No. 20017, NMPC, veg.: Picea, Rubus, Petasites, Caltha, Trollius; Vysoké Tatry Mts, Mengusovce (178), 19.viii.1983, SW, 1M, J. Ježek leg. \& det., slide Inv. No. 19987, NMPC, veg.: Betula, Picea, Caltha, Rumex, Urtica; Nízke Tatry Mts., Baláže (6), 3.vi.1984, SW, 1M, J. Ježek leg. \& det., slide Inv. No. 20477, NMPC, veg.: Fagus, Urtica; Popradská kotlina basin, Skalnatý potok brook env. Tatranská Lomnica (267), 8.vii.1987, SW, 1M, J. Ježek leg. \& det., slide Inv. No. 19957, NMPC, veg.: Picea, Rubus, Petasites, Geum; Oravské Beskydy Mts., Babia hora env. Bystré (4), 1300 m a.l.s., 30.vi.1992, SW, 1M, P. Chvojka leg., J. Ježek det., slide Inv. No. 20325, NMPC; Podbeskydská brázda furrow, Oravské Veselé (218), 29.vi.1992, SW, 1M, P. Chvojka leg., J. Ježek det., slide Inv. No. 20217, NMPC; Kremnické vrchy Mts., Železná Breznica env., brook (390), 29.iv.2012, SW, 1M, J. Oboňa leg., J. Ježek det., slide Inv. No. 20044, NMPC.

Comments: A common species distributed largely in western and central Europe and Scandinavia.

\section{Pneumia nubila (Meigen, 1818)}

Published records: Remetské Hámre (251), Rimavská Sobota (256) (Szabó 1965a,b); Revišské Podhradie (252), Soblahov (273) (Halgoš 1973); Remetské Hámre (251), Revišské Podhradie (252), Rimavská Sobota (256), Soblahov (273) (Halgoš 1984); Nad Šenkárkou (193) (Straka \& Majzlan 2006); Čierna Lehota (37), Žitná - Radiša (394) (Straka \& Majzlan 2007); Danielov dom (43), Štart (289) (Straka \& Majzlan 2009); Dubová (55), Dudváh (58), Súčanka (303) (Straka 2009); Š́r (309) (Straka \& Majzlan 2010a), Bábsky les forest (5) (Straka \& Majzlan 2010b); Šišulákov mlyn mill (266) (Straka \& Majzlan 2013).

Material examined: Košická kotlina basin, Solivar env. Prešov (275), 23.v.1979, SW, 1M, J. Ježek leg. \& det., slide Inv. No. 19966, NMPC; Slanské vrchy Mts., Červenica (33), 24.v.1979, SW, 1M, J. Ježek leg. \& det., slide Inv. No. 20277, NMPC, veg.: Alnus, Corylus, Sorbus, Scirpus, Juncus, Equisetum; Východoslovenská pahorkatina hills, Čaklov (30), 25.v.1979, SW, 1M, J. Ježek leg. \& det., slide Inv. No. 20550, NMPC, veg.: Populus, Juglans, Sambucus, Crataegus, Urtica, Lappa; Slanské vrchy Mts., Juskova Vol'a env., brook (104), 25.v.1979, SW, 1M, J. Ježek leg. \& det., slide Inv. No. 20190, NMPC, veg.: Fagus, Corylus, Carpinus, Rosa, Ranunculus; Košická kotlina basin, Herl’any (72), 26.v.1979, SW, 1M, J. Ježek leg. \& det., slide Inv. No. 20125, NMPC, veg.: Rosa, Rubus, Asperula, Urtica; Laborecká vrchovina hills, Belá nad Cirochou, brook (14), 6.ix.1979, SW, 1M, J. Ježek leg. \& det., slide Inv. No. 20001, NMPC, veg.: Salix, Alnus, Caltha, Urtica; Nízke Tatry Mts., Brusno env. Brezno (24), 16.ix.1985, SW, 1M, J. Ježek leg. \& det., slide Inv. No. 20092, NMPC, veg.: Salix, Alnus, Corylus, Urtica, Asarum; Laborecká vrchovina hills, Krásny Brod env. Medzilaborce, brook, well (128), 10.ix.1991, SW, 1M, J. Ježek leg. \& det., slide Inv. No. 20109, NMPC, veg.: Alnus, Salix, Corylus, Rubus, Caltha, Mentha, Carex, Dipsacus, Musci; Malé Karpaty Mts., Dúbravka (57), 18.viii.2011, SW, 2M, J. Lukáš leg., J. Ježek det., slides Inv. No. 20628 and 20643, NMPC; Malé Karpaty Mts., Mokrý potok brook (184), 210 - 309 m a.s.l., 25.viii.2011, SW, 2M, J. Lukáš leg., J. Ježek det., slides Inv. No. 20621 and 20647, NMPC; Malé Karpaty Mts., Fialkový potok brook (61), 247 m a.s.l., 4.ix.2011, SW, 1M, J. Lukáš leg., J. Ježek det., slide Inv. No. 20634, NMPC; Považský Inovec Mts., Lúka (155), 18.iv.2013, SW, 1M, J. Oboňa leg., J. Ježek det., slide Inv. No. 20078, NMPC; Kremnické vrchy Mts., Železná Breznica env., brook (390), 30.iv.2013, SW, 1M, J. Oboňa leg., J. Ježek det., slide Inv. No. 20057, NMPC; Strážovské vrchy Mts., Diviacka Nová Ves (46), 20.v.2013, 1M, HC, J. Oboňa leg., J. Ježek det., slide Inv. No. 20082, NMPC; Strážovské vrchy 
Mts., Trstenec well env. Diviacka Nová Ves (334), 1.vii.2013, 1M, SW, J. Oboňa leg., J. Ježek det., slide Inv. No. 20223, NMPC.

Comments: A very common species, recorded from throughout Europe and the Canary Islands.

\section{Pneumia palustris (Meigen, 1804)}

Published record: Solivar (275) (Ježek 2006a).

Material examined: Košická kotlina basin, Solivar env. Prešov (275), 23.v.1979, SW, 1M, J. Ježek leg. \& det., slide Inv. No. 19967, NMPC; Slanské vrchy Mts., Juskova Vol’a env., brook (104), 25.v.1979, SW, 1M, J. Ježek leg. \& det., slide Inv. No. 20193, NMPC, veg.: Fagus, Corylus, Carpinus, Rosa, Ranunculus; Čierna hora Mts., Čermel’ nr. Košice (31), 19.v.1980, SW, 1M, J. Ježek leg. \& det., slide Inv. No. 20369, NMPC, veg.: Alnus, Salix, Carpinus, Petasites, Urtica; Čierna hora Mts., Margecany brook (169), 20.v.1980, SW, 1 M, J. Ježek leg. \& det., slide Inv. No. 20273, NMPC, veg.: Mentha, Carex, Urtica; Laborecká vrchovina hills, Starina env., brook (288), 13.viii.2003. SW, 1M, J. Ševčík leg., J. Ježek det., slide Inv. No. 12026, NMPC, veg.: Alnus, Fagus, Carpinus; Malé Karpaty Mts., Mokrý potok brook (184), 210 m a.s.l., 25.viii.2011, SW, 1M, J. Lukáš leg., J. Ježek det., slide Inv. No. 20630, NMPC; Kremnické vrchy Mts., Železná Breznica env., brook (390), 30.iv.2012, SW, 1M, J. Oboňa leg., J. Ježek det., slide Inv. No. 20038, NMPC.

Comments: A species widespread in Europe, the Canary Islands and Turkey.

\section{Pneumia pilularia (Tonnoir, 1940)}

Published records: Šúr (309) (Halgoš 1973, Halgoš 1984); Dunaj river basin (60) (larvae, Bulánková 2003). Material examined: Nízke Tatry Mts., Brusno env. Brezno (24), 16.ix.1985, SW, 1M, J. Ježek leg. \& det., slide Inv. No. 20094, NMPC, veg.: Salix, Alnus, Corylus, Urtica, Asarum; Ondavská vrchovina hills, Kružlová (131), 14.ix.1991, SW, 1M, J. Ježek leg. \& det., slide Inv. No. 20355, NMPC, veg.: Alnus, Salix, Pinus, Pirus, Betula, Rubus, Petasites, Equisetum; Ondavská vrchovina hills, Gerlachov env. Bardejov (65), 17.ix.1991, SW, 1M, J. Ježek leg. \& det., slide Inv. No. 20372, NMPC, veg.: Alnus, Salix, Rubus, Urtica, Poaceae; Čergov Mts., Hromacký vrch env. Livov (88), 830 m a.s.l., 26.ix.1992, SW, 1M, J. Ježek leg. \& det., slide Inv. No. 20577, NMPC, veg.: Fagus, Picea, Pinus, Abies, Betula, Mentha, Poaceae; Čergov Mts., Olejníkov (214), 26.ix.1992, SW, 1M, J. Ježek leg. \& det., slide Inv. No. 20391, NMPC, veg.: Alnus, Rubus, Petasites, Caltha, Urtica, Mentha, Marchantiopsida.

Comments: Relatively sporadic European species.

\section{Pneumia plumicornis (Tonnoir, 1922)}

Published records: Demänovská dolina valley (45), Korytnica (118), Nižné Hágy (201), Tatranská Kotlina (317), Tatranská Lomnica (319), Vyšné Hágy (376) (Szabó 1965a,b); Horný Smokovec (79) (Szabó 1965b); Demänovská dolina valley (45), Horný Smokovec (79), Korytnica (118), Nižné Hágy (201), Tatranská Lomnica (319), Vyšné Hágy (376) (Halgoš 1984); Liptovská Porúbka (142), Ludrová (153), Patočiny (222), Stanišovská dolina valley (282), Stanišovské sedlo saddleback (283) (Ježek 1993); Antalova studnička well (2), Bánovo (7), Čierny Hron brook (39), Dlhý Grúň (47), Hiadel'ské sedlo saddleback (75), Prostredná stream (241), Rakytovo brook (247) (Ježek 1997); Štart (289) (Straka \& Majzlan 2009).

Material examined: Nízke Tatry Mts., Liptovská Lúžna (141), 13.ix.1985, SW, 1M, J. Ježek leg. \& det., slide Inv. No. 20284, NMPC, veg.: Salix, Grossularia, Petasites, Mentha, Urtica; Nízke Tatry Mts., Stanišovské sedlo saddleback nr. Liptovská Porúbka (283), 8.ix.1986, SW, 1M, J. Ježek leg. \& det., slide Inv. No. 19975, NMPC; Čergov Mts., Baranie (10), 25.ix.1992, SW, 1M, J. Ježek leg. \& det., slide Inv. No. 20437, NMPC, veg.: Betula, Pinus, Rubus, Mentha, Equisetum, Caltha, Hypericum, Pteropsida; Čergov Mts., Lysá (159), 1068 m a.s.l., 25.ix.1992, SW, 1M, J. Ježek leg. \& det., slide Inv. No. 20386, NMPC, veg.: Picea, Pinus, Betula, Corylus, Juniperus, Hypericum, Caltha; Čergov Mts., Tokáreň (325), 25.ix.1992, SW, 1F, J. Ježek leg. \& det., slide Inv. No. 20441, NMPC, veg.: Acer, Picea, Fagus, Corylus, Mentha, Caltha; Čergov Mts., Majdan (160), 26.ix.1992, SW, 1M, J. Ježek leg. \& det., slide Inv. No. 20296, NMPC, veg.: Fagus, Urtica, Aruncus, Pteropsida, Musci; Čergov Mts., Olejníkov (214), 26.ix.1992, SW, 1M, J. Ježek leg. \& det., slide Inv. No. 20387, NMPC, veg.: Alnus, Rubus, Petasites, Caltha, Urtica, Mentha, Marchantiopsida; Čergov Mts., Minčol (182), 27.ix.1992, SW, 2M, J. Ježek leg. \& det., slides Inv. No. 20143 and 20319, NMPC, veg.: Picea, Acer, Rubus, Juncus; Malá Fatra Mts., Pivovarský potok brook (228), 1120 m a.s.l., 24.ix.1993, SW, 1M, P. Chvojka leg., J. Ježek det., slide Inv. No. 20124, NMPC.

Comments: A central European species.

\section{Pneumia rivularis Berdén, 1954}

Published records: Hrochotská dolina valley (87), Hrončecký grúň slope (90) (Ježek 2009a). 
Material examined: Podbeskydská brázda, Oravské Veselé (218), 29.vi.1992, SW, 1M, P. Chvojka leg., J. Ježek det., slide Inv. No. 20218, NMPC.

Comments: A rare European species, the assessment of the current conservation status: EN.

\section{Pneumia stammeri (Jung, 1956)}

Published records: Gader (64), Turiec (336), Valča (348) (larvae, Bulánková 1991); Morava river (187) (larvae, Krno et al. 1994); Malá Homôlka (162) (larvae, Bulánková \& Halgoš 1995); Malá Homôlka (162), Vel'ká Homôlka (352) (larvae, Krno et al. 1995); Turiec (336) (larvae, Bulánková \& Degma 1996); Hučava stream (93), Mičová stream (181) (larvae, Bulánková 1999, larvae, Bitušík et al. 2000); Kamenný potok brook (105) (larvae, Bulánková et al. 2001); Gidra stream (67) (larvae, Bulánková 2002); Hron river basin (89), Latorica river basin (137), Morava river basin (186), Slaná river basin (269), upper Váh river basin (345) (larvae, Bulánková 2003); Stupavský potok brook (300) (larvae, Bulánková 2007); Val'kovňa (349) (larvae, Bulánková \& Némethová 2007); Hluboký potok brook (76), Stužická rieka stream (302) (larvae, Novikmec et al. 2007); Horáreň Sliače (78), Hriňová (86), Hrušovo (92), Jakubany (97), Kl’ačno (113), Liptovská Teplička (143), Majdan (160), Monkova dolina valley (185), Poráč (257), Rajecká Lesná (245), Revúčka (254), Senné (265), Šuja (307), Tužina (337), Vel'ký Dvor (357), Vernár (360), Vrátna dolina valley (370), Vyšná Maša (375), Zimovci (393), Železná studnička well (391) (larvae, Mišíková Elexová 2010); Vydrica stream (373) (larvae, Števove \& Bulánková 2010).

Material examined: Slanské vrchy Mts., Lúčina (152), 24.v.1979, SW, 1M, J. Ježek leg. \& det., slide Inv. No. 20257, NMPC, veg.: Betula, Alnus, Mentha, Ajuga, Scirpus; Východoslovenská pahorkatina hills, Zámutov env. Vranov nad Topl'ou, brook (384), 24.v.1979, SW, 1M, J. Ježek leg. \& det., slide Inv. No. 20104, NMPC, veg.: Fagus, Betula, Mentha, Urtica; Čierna hora Mts., Čermel’ nr. Košice (31), 19.v.1980, SW, 1M, J. Ježek leg. \& det., slide Inv. No. 20368, NMPC, veg.: Alnus, Salix, Carpinus, Petasites, Urtica; Čierna hora Mts., Kavečany, brook (109), 19.v.1980, SW, 1M, J. Ježek leg. \& det., slide Inv. No. 20201, NMPC, veg.: Fagus, Salix, Alnus, Petasites, Urtica; Popradská kotlina basin, Tatranská Kotlina env. Belianska jaskyňa cave, brook (317), 18.vi.1982, SW, 1M, J. Ježek leg. \& det., slide Inv. No. 19973, NMPC, veg.: Picea, Petasites, Musci; Liptovská kotlina basin, Pavčina Lehota nr. Liptovský Mikuláš (223), 10.vi.1987, SW, 1M, J. Ježek leg. \& det., slide Inv. No. 19946, NMPC, veg.: Salix, Rubus, Caltha, Alisma, Filipendula, Geum, Urtica.

Comments: Quite a rare European species. In Slovakia relatively common. Until now, known from Slovakia only as larvae, this is first adult records of this species in Slovakia. The assessment of the current conservation status in contrast to the Czech Republic is problematic (a monitoring in future will be probably needed).

\section{Pneumia trivialis (Eaton, 1893)}

Published records: Bardejov (11), Oravský Podzámok (219), Rimavská Sobota (256), Tisovec (324), Zbojská (388) (Szabó 1965a,b); Bardejov (11), Oravký Podzámok (219), Rimavská Sobota (256), Tisovec (324), Zbojská (388) (Halgoš 1984); Baláže (6), Dúbrava (56), Hiadel'ská dolina valley (74), Hiadel’ské sedlo saddleback (75), Il’anovo (94), Kochul'a (114), Kropácová (130), Liptovská Porúbka (142), Liptovské Kl'ačany (144), Liptovský Hrádok (146), Pavčina Lehota (223), Ploštín (229), Prostredná stream (241), Sopotnická dolina valley (278), Strmý vrštek hill (298), Tále (316) (Ježek 1993); Turiec (336) (larvae Bulánková \& Degma 1996); Bodrog river basin (18), Hron river basin (89), Morava river basin (186), upper Váh river basin (345), Slaná river basin (269) (larvae, Bulánková 2003); Vyšné Račkové pleso mountain lake (377) (larvae, Bulánková \& Zat'ovičová 2006); Zadná Pol'ana (382) (Ježek 2009a); Jamy (98) (Straka \& Majzlan 2009); Stavanie (290) (larvae, Mišíková Elexová 2010).

Material examined: Košická kotlina basin, Solivar env. Prešov (275), 23.v.1979, SW, 1M, J. Ježek leg. \& det., slide Inv. No. 19968, NMPC; Slanské vrchy Mts., Červenica (33), 24.v.1979, SW, 1M, J. Ježek leg. \& det., slide Inv. No. 20275, NMPC, veg.: Alnus, Corylus, Sorbus, Scirpus, Juncus, Equisetum; Slanské vrchy Mts., Lúčina (152), 24.v.1979, SW, 1M, J. Ježek leg. \& det., slide Inv. No. 20256, NMPC, veg.: Betula, Alnus, Mentha, Ajuga, Scirpus; Slanské vrchy Mts., Tuhrina (335), 24.v.1979, SW, 1M, J. Ježek leg. \& det., slide Inv. No. 20176, NMPC, veg.: Fagus, Carpinus, Alnus, Viola; Východoslovenská pahorkatina hills, Zámutov env. Vranov nad Topl'ou, brook (384), 24.v.1979, SW, 1M, J. Ježek leg. \& det., slide Inv. No. 20105, NMPC, veg.: Fagus, Betula, Mentha, Urtica; Slanské vrchy Mts., Banské (8), 25.v.1979, SW, 1M, J. Ježek leg. \& det., slide Inv. No. 20548, NMPC, veg.: Picea, Rubus, Myosotis; Slanské vrchy Mts., Juskova Vol'a env., brook (103), 25.v.1979, SW, 1M, J. Ježek leg. \& det., slide Inv. No. 20191, NMPC, veg.: Fagus, Corylus, Carpinus, Rosa, Ranunculus; Košická kotlina basin, Herl’any (73), 26.v.1979, SW, 1M, J. Ježek leg. \& det., slide Inv. No. 20127, NMPC, veg.: Rosa, Rubus, Asperula, Urtica; Košická kotlina basin, Košický Klečenov (124), 26.v.1979, SW, 1M, J. Ježek leg. \& det., slide Inv. No. 20431, NMPC, veg.: Fagus, Asarum, Trifolium, Asperula; Košická kotlina basin, Nižná Kamenica (198), 800 m a.s.l., 26.v.1979, SW, 1M, J. Ježek leg. \& det., slide Inv. No. 20422, NMPC, veg.: Fagus, Acer, Ranunculus, Asarum, Pteropsida; Košická kotlina basin, Vyšná Kamenica (374), 26.v.1979, SW, 1M, J. Ježek leg. \& det., slide Inv. No. 20254, NMPC, veg.: Fagus, Acer, Impatiens, 
Asperula, Pteropsida; Laborecká vrchovina hills, Belá nad Cirochou, brook (14), 6.ix.1979, SW, 1M, J. Ježek leg. \& det., slide Inv. No. 20002, NMPC, veg.: Salix, Alnus, Caltha, Urtica; Vihorlatské vrchy Mts., Zemplínske Hámre, brook (392), 6.ix.1979, SW, 1M, J. Ježek leg. \& det., slide Inv. No. 20335, NMPC, veg.: Salix, Alnus, Urtica; Bukovské vrchy Mts., Uličské Krivé (343), 7.ix.1979, SW, 1M, J. Ježek leg. \& det., slide Inv. No. 20617, NMPC, veg.: Alnus, Salix, Scirpus; Bukovské vrchy Mts., Zboj (385), 7.ix.1979, SW, 1M, J. Ježek leg. \& det., slide Inv. No. 20528, NMPC, veg.: Alnus, Corylus, Humulus, Mentha; Laborecká vrchovina hills, Nižná Čabina (197), 9.ix.1979. SW, 1M, J. Ježek leg. \& det., slide Inv. No. 20306, NMPC, veg.: Salix, Alnus, Urtica, Phragmites; Čierna hora Mts., Čermel' nr. Košice (31), 19.v.1980, SW, 1M, J. Ježek leg. \& det., slide Inv. No. 20362, NMPC, veg.: Alnus, Salix, Carpinus, Petasites, Urtica; Čierna hora Mts., Margecany, brook (169), 20.v.1980, SW, 1M, J. Ježek leg. \& det., slide Inv. No. 20271, NMPC, veg.: Mentha, Carex, Urtica; Čierna hora Mts., Kysak (135), 600 m a.s.l., 19.v.1980, SW, 1M, J. Ježek leg. \& det., slide Inv. No. 20168, NMPC, veg.: Fagus, Carpinus, Juncus, Mentha; Popradská kotlina basin, Tatranská Kotlina (317), 16.vi.1982, SW, 1M, J. Ježek leg. \& det., slide Inv. No. 19991, NMPC, veg.: Picea, Caltha; Popradská kotlina basin, Tatranská Polianka (320), 21.viii.1983, SW, 1M, J. Ježek leg. \& det., slide Inv. No. 20342, NMPC. veg.: Picea, Alnus, Rubus, Caltha, Lysimachia, Filipendula, Urtica; Popradská kotlina basin, Dolný Smokovec (53), 22.viii.1983, SW, 1M, J. Ježek leg. \& det., slide Inv. No. 20203, NMPC, veg.: Alnus, Filipendula, Caltha, Urtica; Vysoké Tatry Mts., Biely Váh (17), 16.8.1983, SW, 1M, J. Ježek leg. \& det., slide Inv. No. 20384, NMPC, veg.: Picea, Rubus, Sphagnum, Scirpus, Juncus, Caltha; Popradská kotlina basin, Batizovce env., brook (13), 19.viii.1983, SW, 1M, J. Ježek leg. \& det., slide Inv. No. 19976, NMPC, veg.: Alnus, Grossularia, Caltha; Vysoké Tatry Mts, Mengusovce (178), 19.viii.1983, SW, 1M, J. Ježek leg. \& det., slide Inv. No. 20019, NMPC, veg.: Betula, Picea, Caltha, Rumex, Urtica; Popradská kotlina basin, Tatranská Lesná (318), 23.viii.1983, SW, 1M, J. Ježek leg. \& det., slide Inv. No. 20544, NMPC, veg.: Alnus, Scirpus, Filipendula, Urtica; Nízke Tatry Mts., Brusno env. Brezno (24), 16.ix.1985, SW, 1M, J. Ježek leg. \& det., slide Inv. No. 20095, NMPC, veg.: Salix, Alnus, Corylus, Urtica, Asarum; Liptovská kotlina basin, Pavčina Lehota nr. Liptovský Mikuláš (223), 10.ix.1987, SW, 1M, J. Ježek leg. \& det., slide Inv. No. 19948, NMPC, veg.: Salix, Rubus, Caltha, Alisma, Filipendula, Geum, Urtica; Rožňavská kotlina basin, Rožňava (257), 13.vii.1989, SW, 1M, J. Ježek leg. \& det., slide Inv. No. 20404, NMPC, veg.: Alnus, Carpinus, Juncus, Impatiens, Pteropsida; Popradská kotlina basin, Nová Lesná (206), 28.ix.1989, SW, 1M, P. Chvojka leg., J. Ježek det., slide Inv. No. 20251, NMPC; Popradská kotlina basin, Červený potok brook - Nová Lesná (34), 22.vii.1990, SW, 1M, P. Chvojka leg., J. Ježek det., slide Inv. No. 20410, NMPC; Laborecká vrchovina hills, Krásny Brod env. Medzilaborce, brook, well (128), 10.ix.1991, SW, 1M, J. Ježek leg. \& det., slide Inv. No. 20113, NMPC, veg.: Alnus, Salix, Corylus, Rubus, Caltha, Mentha, Carex, Dipsacus, Musci; Laborecká vrchovina hills, Malá Pol’ana (164), 10.ix.1991, SW, 1M, J. Ježek leg. \& det., slide Inv. No. 20348, NMPC, veg.: Fagus, Carpinus, Corylus, Rubus, Melampyrum, Daucaceae; Ondavská vrchovina hills, Bukovce (25), 11.ix.1991, SW, 1M, J. Ježek leg. \& det., slide Inv. No. 20148, NMPC, veg.: Salix, Alnus, Acer, Rubus, Humulus, Petasites, Lappa, Urtica; Ondavská vrchovina hills, Nová Polianka env. Svidník (207), 12.ix.1991, SW, 1M, J. Ježek leg. \& det., slide Inv. No. 20378, NMPC, veg.: Salix, Alnus, Corylus, Rubus, Typha, Lappa, Urtica, Daucaceae; Ondavská vrchovina hills, Rakovčík (246), 12.ix.1991, SW, 1F, J. Ježek leg. \& det., slide Inv. No. 20291, NMPC, veg.: Alnus, Prunus, Corylus, Sambucus, Salix, Cirsium, Poaceae, Daucaceae; Laborecká vrchovina hills, Korejovce (117), 13.ix.1991, SW, 1M, J. Ježek leg. \& det., slide Inv. No. 20161, NMPC, veg.: Salix, Alnus, Petasites, Dipsacus, Impatiens, Musci, Poaceae; Ondavská vrchovina hills, Nižná Pisaná brook (200), 14.ix.1991, SW, 1M, J. Ježek leg. \& det., slide Inv. No. 20208, NMPC, veg.: Alnus, Salix, Carpinus, Geranium, Artemisia, Urtica, Pteropsida, Poaceae; Ondavská vrchovina hills, Šarišské Čierne (263), 15.ix.1991, SW, 1M, J. Ježek leg. \& det., slide Inv. No. 20182, NMPC, veg.: Salix, Alnus, Pirus, Frangula, Evonymus, Rubus, Urtica, Daucaceae; Čergov Mts., Olejníkov (214), 26.ix.1992, SW, 1M, J. Ježek leg. \& det., slide Inv. No. 20389, NMPC, veg.: Alnus, Rubus, Petasites, Caltha, Urtica, Mentha, Marchantiopsida; Spišsko-šarišské medzihorie Imts., Stará Lubbovňa (284), 28.ix.1992, SW, 1M, J. Ježek leg. \& det., slide Inv. No. 20394, NMPC, veg.: Salix, Alnus, Urtica, Lappa; Muránska planina Mts., Muránska planina plain (191), 22.vii.1999, SW, 1M, P. Chvojka leg., J. Ježek det., slide Inv. No. 20299, NMPC; Bukovské vrchy Mts., Stinská slatinka meadow (291), 700 m a.s.l., 20.vii.2005, SW, 1M, P. Kment leg., J. Ježek det., slide Inv. No. 20436, NMPC; Podunajská rovina flat, Šúr (309), 7.viii.2009, MT, 1M, J. Lukáš leg., J. Ježek det., slide Inv. No. 20637, NMPC; Malé Karpaty Mts., Mokrý potok brook (183), 210 m a.s.l., 25.viii.2011, SW, 1M, J. Lukáš leg., J. Ježek det., slide Inv. No. 20644, NMPC; Kremnické vrchy Mts., Železná Breznica env., brook (390), 29.-30.iv.2012 and 30.iv.2013, 3M, all SW, J. Oboňa leg., J. Ježek det., slides Inv. No. 20049, 20040 and 20056, NMPC; Pol'ana Mts., Hučava stream (93), SW, 6.v.2013, 1M, J. Oboňa leg., J. Ježek det., slide Inv. No. 20062, NMPC.

Comments: A very common European species. 


\section{Saraiella Vaillant, 1973}

\section{Saraiella parva (Vaillant, 1963)}

Published record: Pilsko (227) (Ježek 2006a).

Material examined: Vysoké Tatry Mts., Suchý vrch hill., Javorina (306), 26.vi.1982, SW, 1M, J. Ježek leg. \& det., slide Inv. No. 20013, NMPC, veg.: Picea, Rubus, Petasites, Caltha, Trollius.

Comments: Relatively sporadic European species, the assessment of the current conservation status: CR.

\section{Saraiella rotunda (Krek, 1970)}

Published record: Gerlachov (66) (Ježek 2006a).

Material examined: Slanské vrchy Mts., Tuhrina (335), 24.v.1979, SW, 1M, J. Ježek leg. \& det., slide Inv. No. 20174, NMPC, veg.: Fagus, Carpinus, Alnus, Viola; Vysoké Tatry Mts., Holica (77), 1630 m a.s.l., 26.vi.1982, SW, 1M, J. Ježek leg. \& det., slide Inv. No. 20131, NMPC, veg.: Picea, Petasites, Musci; Nízke Tatry Mts., Liptovská Lúžna (141), 13.ix.1985, SW, 1F, J. Ježek leg. \& det., slide Inv. No. 20470, NMPC, veg.: Picea, Mentha, Urtica; Popradská kotlina basin, Skalnatý potok brook env. Tatranská Lomnica (267), 8.vii.1987, SW, 1M, J. Ježek leg. \& det., slide Inv. No. 19958, NMPC, veg.: Picea, Rubus, Petasites, Geum.

Comments: Relatively sporadic European species, the assessment of the current conservation status: CR.

\section{Szaboiella Vaillant, 1979}

\section{Szaboiella hibernica (Tonnoir, 1940)}

Published record: Hrončecký grúň slope (90) (Ježek 2009a).

Comments: Relatively rare European species, the assessment of the current conservation status: CR.

\section{Tonnoiriella Vaillant, 1972}

\section{Tonnoiriella nigricauda (Tonnoir, 1919)}

Published record: Mengusovce (178) (Ježek 2006a).

Material examined: Bukovské vrchy Mts., Uličské Krivé (343), 7.ix.1979, SW, 1M, J. Ježek leg. \& det., slide Inv. No. 20619, NMPC, veg.: Alnus, Salix, Scirpus; Vysoké Tatry Mts, Mengusovce (178), 19.viii.1983, SW, 1M, J. Ježek leg. \& det., slide Inv. No. 20018, NMPC, veg.: Betula, Picea, Caltha, Rumex, Urtica; Popradská kotlina basin, Tatranská Polianka (320), 21.viii.1983, SW, 1M, J. Ježek leg. \& det., slide Inv. No. 20344, NMPC, veg.: Picea, Alnus, Rubus, Caltha, Lysimachia, Filipendula, Urtica; Liptovská kotlina basin, Pavčina Lehota nr. Liptovský Mikuláš (223), 10.vi.1987, SW, 1M, J. Ježek leg. \& det., slide Inv. No. 19945, NMPC, veg.: Salix, Rubus, Caltha, Alisma, Filipendula, Geum, Urtica; Popradská kotlina basin, Lučivná - Sviniarka env., well and brook (153), 938 m a.s.l., 13.vii.1987, SW, 1M, J. Ježek leg. \& det., slide Inv. No. 19983, NMPC, veg.: Picea, Alnus, Caltha, Filipendula, Equisetum.

Comments: Relatively rare European species, the assessment of the current conservation status: CR.

\section{Tonnoiriella pulchra (Eaton, 1893)}

Published records: Tisovec (324) (Szabó 1960, 1965a,b, Halgoš 1984); Latorica river basin (137), Slaná river basin (269) (larvae, Bulánková 2003); Stupavský potok brook (300) (larvae, Bulánková 2007); Hluboký potok brook (76) (larvae, Novikmec et al. 2007); Val'kovňa (349) (larvae, Mišíková Elexová 2010); Šišulákov mlyn mill (266) (Straka \& Majzlan 2013).

Comments: A European species, only ostensible common.

\section{Tonnoiriella sieberti Wagner, 1993}

Published record: Nižný Komárnik (203) (Ježek 2006a).

Material examined: Košická kotlina basin, Solivar env. Prešov (275), 23.v.1979, SW, 1M, J. Ježek leg. \& det., slide Inv. No. 19965, NMPC; Veporské vrchy Mts., Príslop (240), 8.ix.1979, SW, 1M, J. Ježek leg. \& det., slide Inv. No. 20266, NMPC, veg.: Alnus, Juncus, Mentha, Carex; Čierna hora Mts., Kysak (135), 600 m a.s.l., 19.v.1980, SW, 1M, J. Ježek leg. \& det., slide Inv. No. 20169, NMPC, veg.: Fagus, Carpinus, Juncus, Mentha; Čierna hora Mts., Margecany brook (169), 20.v.1980, SW, 1M, J. Ježek leg. \& det., slide Inv. No. 20270, NMPC, veg.: Mentha, Carex, Urtica; Nízke Tatry Mts., Pohronský Bukovec (235), 18.ix.1985, SW, 1M, J. Ježek leg. \& det., slide Inv. No. 20012, NMPC, veg.: Alnus, Caltha, Filipendula; Kremnické vrchy Mts., 
Železná Breznica env., brook (390), 30.iv.2012, SW, 1M, J. Oboňa leg., J. Ježek det., slide Inv. No. 20042, NMPC

Comments: Generally a rare species, known from Europe and Syria. The assessment of the current conservation status: EN.

\section{Ulomyia Walker, 1856}

\section{Ulomyia annulata annulata (Tonnoir, 1919)}

Published records: Bardejov (11) (Szabó 1960, 1965a, Halgoš 1984); Bardejov (11), Vlkolinec (364) (Szabó 1965b); Liptovské Kl'ačany (144) (Ježek 1993).

Material examined: Čierna hora Mt., Margecany brook (169), 20.v.1980, SW, 1M, J. Ježek leg. \& det., slide Inv. No. 20272, NMPC, veg.: Mentha, Carex, Urtica.

Comments: Not quite common Europaen and western Siberia species.

\section{Ulomyia cognata (Eaton, 1893)}

Published records: Tisovec (324), Zbojská (388) (Szabó 1960, 1965b); Tisovec (324), Zbojská (388) (Szabó 1972-73, Halgoš 1984); Hrochotská dolina valley (87) (Ježek 2009a).

Material examined: Slanské vrchy Mts., Červenica (33), 24.v.1979, SW, 1M, J. Ježek leg. \& det., slide Inv. No. 20280, NMPC, veg.: Alnus, Corylus, Sorbus, Scirpus, Juncus, Equisetum; Slanské vrchy Mts., Tuhrina (335), 24.v.1979, SW, 1M, J. Ježek leg. \& det., slide Inv. No. 20175, NMPC, veg.: Fagus, Carpinus, Alnus, Viola; Čierna hora Mts., Čermel’ nr. Košice (30), 19.v.1980, SW, 1M, J. Ježek leg. \& det., slide Inv. No. 20370, NMPC, veg.: Alnus, Salix, Carpinus, Petasites, Urtica; Popradská kotlina basin, Tatranská Kotlina (317), 16.vi.1982, SW, 1M, J. Ježek leg. \& det., slide Inv. No. 19989, NMPC, veg.: Picea, Caltha; Vysoké Tatry Mts., Holica (77), 1630 m a.s.l., 26.vi.1982, SW, 1M, J. Ježek leg. \& det., slide Inv. No. 20133, NMPC, veg.: Picea, Petasites, Musci; Nízke Tatry Mts., Baláže (6), 3.vi.1984, SW, 1M, J. Ježek leg. \& det., slide Inv. No. 20420, NMPC, veg.: Fagus, Urtica; Laborecká vrchovina hills, Malá Pol’ana (164), 10.ix.1991, SW, 1M, J. Ježek leg. \& det., slide Inv. No. 20350, NMPC, veg.: Fagus, Carpinus, Corylus, Rubus, Melampyrum, Daucaceae; Kremnické vrchy Mts., Železná Breznica env., brook (390), 30.iv.2012, SW, 1M, J. Oboňa leg., J. Ježek det., slide Inv. No. 20041, NMPC.

Comments: A common Europaen species.

\section{Ulomyia fuliginosa (Meigen, 1804)}

Published records: Oravský Podzámok (219), Remetské Hámre (251), Tisovec (324), Vlkolinec (364), Zbojská (388) (Szabó 1965a,b); Zádiel (380) (larvae, Rozkošný 1971); Malacky (167), Revištské Podhradie (252), Soblahov (273) (Halgoš 1973); Malacky (167), Oravský Podzámok (219), Revištské Podhradie (252), Ružomberok (261), Soblahov (273), Tisovec (324), Zádiel (380), Zbojská (388) (Halgoš 1984); Baláže (6), Bukovecká dolina valley (28), Bystrá (29), Hiadel'ská dolina valley (74), Hiadel'ské sedlo saddleback (75), Kochul'a (114), Kropácová (130), Liptovský Hrádok (146), Piesky (224), Ploštín (229), Pohronský Bukovec (235), Prostredná stream (241), Sopotnická dolina valley (278), Staré Hory (287), Strmý vrštek hill (298), Špania Dolina (280), Tále (316) (Ježek 1993); Dunaj river basin (60), Hron river basin (89), lower Váh river basin (149), Morava river basin (186), Slaná river basin (269), upper Váh river basin (345) (larvae, Bulánková 2003); Hrončecký grúň slope (90) (Ježek 2009a); Šišulákov mlyn mill (266) (Straka \& Majzlan 2013).

Material examined: Košická kotlina basin, Solivar env. Prešov, swamps (275), 23.v.1979, SW, 1M, J. Ježek leg. \& det., slide Inv. No. 19969, NMPC; Slanské vrchy Mts., Červenica (33), 24.v.1979, SW, 1M, J. Ježek leg. \& det., slide Inv. No. 20274, NMPC, veg.: Alnus, Corylus, Sorbus, Scirpus, Juncus, Equisetum; Slanské vrchy Mts., Tuhrina (335), 24.v.1979, SW, 1M, J. Ježek leg. \& det., slide Inv. No. 20172, NMPC, veg.: Fagus, Carpinus, Alnus, Viola; Východoslovenská pahorkatina hills, Zámutov env., Vranov nad Toplou, brook (384), 24.v.1979, SW, 1M, J. Ježek leg. \& det., slide Inv. No. 20103, NMPC, veg.: Fagus, Betula, Mentha, Urtica; Košická kotlina basin, Herl’any (73), 26.v.1979, SW, 1M, J. Ježek leg. \& det., slide Inv. No. 20126, NMPC, veg.: Rosa, Rubus, Asperula, Urtica; Košická kotlina basin, Nižná Kamenica (198), 800 m a.s.l., 26.v.1979, SW, 1M, J. Ježek leg. \& det., slide Inv. No. 20421, NMPC, veg.: Fagus, Acer, Ranunculus, Asarum, Pteropsida; Čierna hora Mts., Čermel' nr. Košice (31), 19.v.1980, SW, 1M, J. Ježek leg. \& det., slide Inv. No. 20363, NMPC, veg.: Alnus, Salix, Carpinus, Petasites, Urtica; Čierna hora Mts., Kavečany brook (109), 19.v.1980, SW, 1M, J. Ježek leg. \& det., slide Inv. No. 20199, NMPC, veg.: Fagus, Salix, Alnus, Petasites, Urtica; Čierna hora Mts., Margecany brook (169), 20.v.1980, SW, 1M, J. Ježek leg. \& det., slide Inv. No. 20268, NMPC, veg.: Mentha, Carex, Urtica; Popradská kotlina basin, Batizovce env., brook (13), 19.viii.1983, SW, 1M, J. Ježek leg. \& det., slide Inv. No. 19977, NMPC, veg.: Alnus, Grossularia, Caltha; Vysoké Tatry Mts, Mengusovce (178), 19.viii.1983, SW, 1M, J. Ježek leg. \& det., slide Inv. No. 19986, NMPC, veg.: Betula, Picea, Caltha, Rumex, 
Urtica; Popradská kotlina basin, Tatranská Polianka (320), 21.viii.1983, SW, 1M, J. Ježek leg. \& det., slide Inv. No. 20343, NMPC, veg.: Picea, Alnus, Rubus, Caltha, Lysimachia, Filipendula, Urtica; Popradská kotlina basin, Dolný Smokovec (53), 22.viii.1983, SW, 1M, J. Ježek leg. \& det., slide Inv. No. 20204, NMPC, veg.: Alnus, Filipendula, Caltha, Urtica; Starohorské vrchy Mts., Stará Pila (284), 1.vi.1984., SW, 1M, J. Ježek leg. \& det., slide Inv. No. 20303, NMPC, veg.: Rubus, Carex, Juncus, Urtica, Musci; Nízke Tatry Mts., Baláže (6), 3.vi.1984, SW, 1M, J. Ježek leg. \& det., slide Inv. No. 20476, NMPC, veg.: Fagus, Urtica; Nízke Tatry Mts., Liptovská Lúžna (141), 13.ix.1985, SW, 2M, J. Ježek leg. \& det., slides Inv. No. 20282 and 20469, NMPC, veg.: Salix, Grossularia, Petasites, Mentha, Urtica; Nízke Tatry Mts., Brusno env. Brezno (24), 16.ix.1985, SW, 1M, J. Ježek leg. \& det., slide Inv. No. 20091, NMPC, veg.: Salix, Alnus, Corylus, Urtica, Asarum; Stolické vrchy Mts., Muránska Zdychava (192), 19.vii.1989, SW, 1M, J. Ježek leg. \& det., slide Inv. No. 20139, NMPC, veg.: Alnus, Rubus, Filipendula, Impatiens, Caltha; Laborecká vrchovina hills, Krásny Brod env. Medzilaborce, brook, well (128), 10.ix.1991, SW, 1M, J. Ježek leg. \& det., slide Inv. No. 20108, NMPC, veg.: Alnus, Salix, Corylus, Rubus, Caltha, Mentha, Carex, Dipsacus, Musci; Ondavská vrchovina hills, Makovce (161), 10.ix.1991, SW, 1M, J. Ježek leg. \& det., slide Inv. No. 20446, NMPC, veg.: Alnus, Salix, Rubus, Polygonum, Lappa, Urtica, Poaceae; Laborecká vrchovina hills, Malá Pol'ana (164), 10.ix.1991, SW, 1M, J. Ježek leg. \& det., slide Inv. No. 20347, NMPC, veg.: Fagus, Carpinus, Corylus, Rubus, Melampyrum, Daucaceae; Ondavská vrchovina hills, Rakovčík (246), 12.ix.1991, SW, 1F, J. Ježek leg. \& det., slide Inv. No. 20290, NMPC, veg.: Alnus, Prunus, Corylus, Sambucus, Salix, Cirsium, Poaceae, Daucaceae; Laborecká vrchovina hills, Medvedzie (175), 13.ix.1991, SW, 1M, J. Ježek leg. \& det., slide Inv. No. 20298, NMPC, veg.: Carpinus, Fagus, Corylus, Rubus, Urtica; Ondavská vrchovina hills, Kružlová (131), 14.ix.1991, SW, 1M, J. Ježek leg. \& det., slide Inv. No. 20354, NMPC, veg.: Alnus, Salix, Pinus, Pirus, Betula, Rubus, Petasites, Equisetum; Ondavská vrchovina hills, Šarišské Čierne (263), 15.ix.1991, SW, 1M, J. Ježek leg. \& det., slide Inv. No. 20181, NMPC, veg.: Salix, Alnus, Pirus, Frangula, Evonymus, Rubus, Urtica, Daucaceae; Čergov Mts., Olejníkov (214), 26.ix.1992, SW, 2M, J. Ježek leg. \& det., slides Inv. No. 20388 and 20498, NMPC, veg.: Alnus, Rubus, Petasites, Caltha, Urtica, Mentha, Marchantiopsida; Spišsko-šarišské medzihorie Imts., Stará Lubovňa (284), 28.ix.1992, SW, 1M, J. Ježek leg. \& det., slide Inv. No. 20393, NMPC, veg.: Salix, Alnus, Urtica, Lappa; Muránska planina Mts., Muránska planina plain env. Kačkava (191), 16.vi.1999, SW, 1M, P. Chvojka leg., J. Ježek det., slide Inv. No. 20261, NMPC; Bukovské vrchy Mts., Nová Sedlica (207), 9.v.-3.vii. 2002, YPT, 1M, P. Kment leg., J. Ježek det., slide Inv. No. 20312, NMPC; Laborecká vrchovina hills, Starina env., brook (288), 13.vii.2003, SW, 1M, J. Ševčík leg., J. Ježek det., slide Inv. No. 12028, NMPC, veg.: Alnus, Fagus, Carpinus; Malé Karpaty Mts., Dúbravka (57), 18.viii.2011, SW, 2M, J. Lukáš leg., J. Ježek det., slides Inv. No. 20626 and 20642, NMPC; Malé Karpaty Mts., Mokrý potok brook (184), 210 - 282 m a.s.l., 25.viii.2011, SW, 2M, J. Lukáš leg., J. Ježek det., slides Inv. No. 20622 and 20648, NMPC; Malé Karpaty Mts., Fialkový potok brook (62), 247 m a.s.l., 4.ix.2011, SW, 1M, J. Lukáš leg., J. Ježek det., slide Inv. No. 20633, NMPC; Kremnické vrchy Mts., Železná Breznica env., brook (390 - Fig. 11), 29.-30.iv.2012, 2M, all SW, J. Oboňa leg., J. Ježek det., slides Inv. No. 20039 and 20045, NMPC; Strážovské vrchy Mts., Trstenec well env. Diviacka Nová Ves (334 - Fig. 10), 6.v.2013, 1M, SW, J. Oboňa leg., J. Ježek det., slide Inv. No. 20065, NMPC. Comments: The most common European species.

\section{Ulomyia vaseki Ježek, 2002}

Published record: Stará Voda (286) (Ježek 2002).

Material examined: Vysoké Tatry Mts., Holica (77), 1630 m a.s.l., 26.vi.1982, SW, 1M, J. Ježek leg. \& det., slide Inv. No. 20130, NMPC, veg.: Picea, Petasites, Musci; Vysoké Tatry Mts., Suchý vrch env. Javorina (306), 26.vi.1982, SW, 1M, J. Ježek leg. \& det., slide Inv. No. 20015, NMPC, veg.: Picea, Rubus, Petasites, Caltha, Trollius; Vysoké Tatry Mts., Vel'ká Pálenica (353), 15.viii.1983, SW, 1M, J. Ježek leg. \& det., slide Inv. No. 20395, NMPC, veg.: Sorbus, Acer, Picea, Petasites; Liptovská kotlina basin, Tri Studničky env. Pavučia dolina valley (331), 16.viii.1983, SW, 1M, J. Ježek leg. \& det., slide Inv. No. 20415, NMPC, veg.: Picea, Caltha, Urtica; Popradská kotlina basin, Skalnatý potok brook env. Tatranská Lomnica (267), 8.vii.1987, SW, 1M, J. Ježek leg. \& det., slide Inv. No. 19955, NMPC, veg.: Picea, Rubus, Petasites, Geum; Podtatranská kotlina basin, Kežmarská Biela Voda (110), 9.vii.1987, SW, 1M, J. Ježek leg. \& det., slide Inv. No. 20333, NMPC, veg.: Sorbus, Pinus mugo, Rubus, Caltha, Pteropsida; Revúcka vrchovina Mts., Rejdová (250), 18.vii.1989, SW, 1M, J. Ježek leg. \& det., slide Inv. No. 20478, NMPC, veg.: Picea, Fagus, Salix, Sambucus, Caltha, Scirpus, Daucaceae; Podbeskydská brázda furrow, Oravská Polhora env. Borsučie (217), 30.vi.1992, SW, 1M, P. Chvojka leg., J. Ježek det., slide Inv. No. 20252, NMPC; Chočské vrchy Mts., Valaská Dubová, well (347), 4.vii.1992, SW, 1M, P. Chvojka leg., J. Ježek det., slide Inv. No. 20197, NMPC; Muránska planina Mts., Havraník (72), 15.vi.1999, SW, 1M, P. Chvojka leg., J. Ježek det., slide Inv. No. 19960, NMPC.

Comments: Relatively rare central European species. The assessment of the current conservation status: EN. 


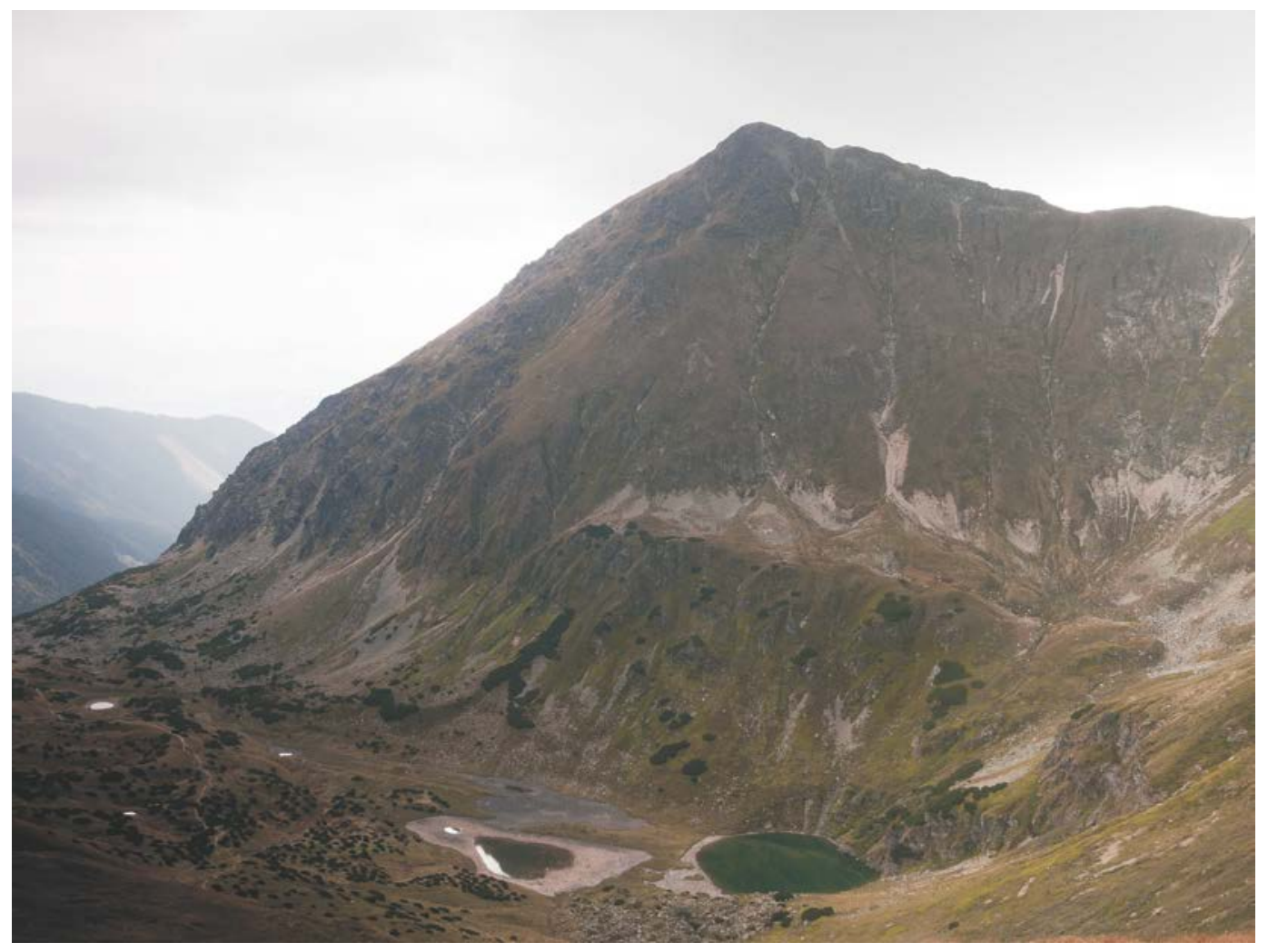

Fig 9: Račková dolina valley, mountain lakes (photo by Miloš Villaris).

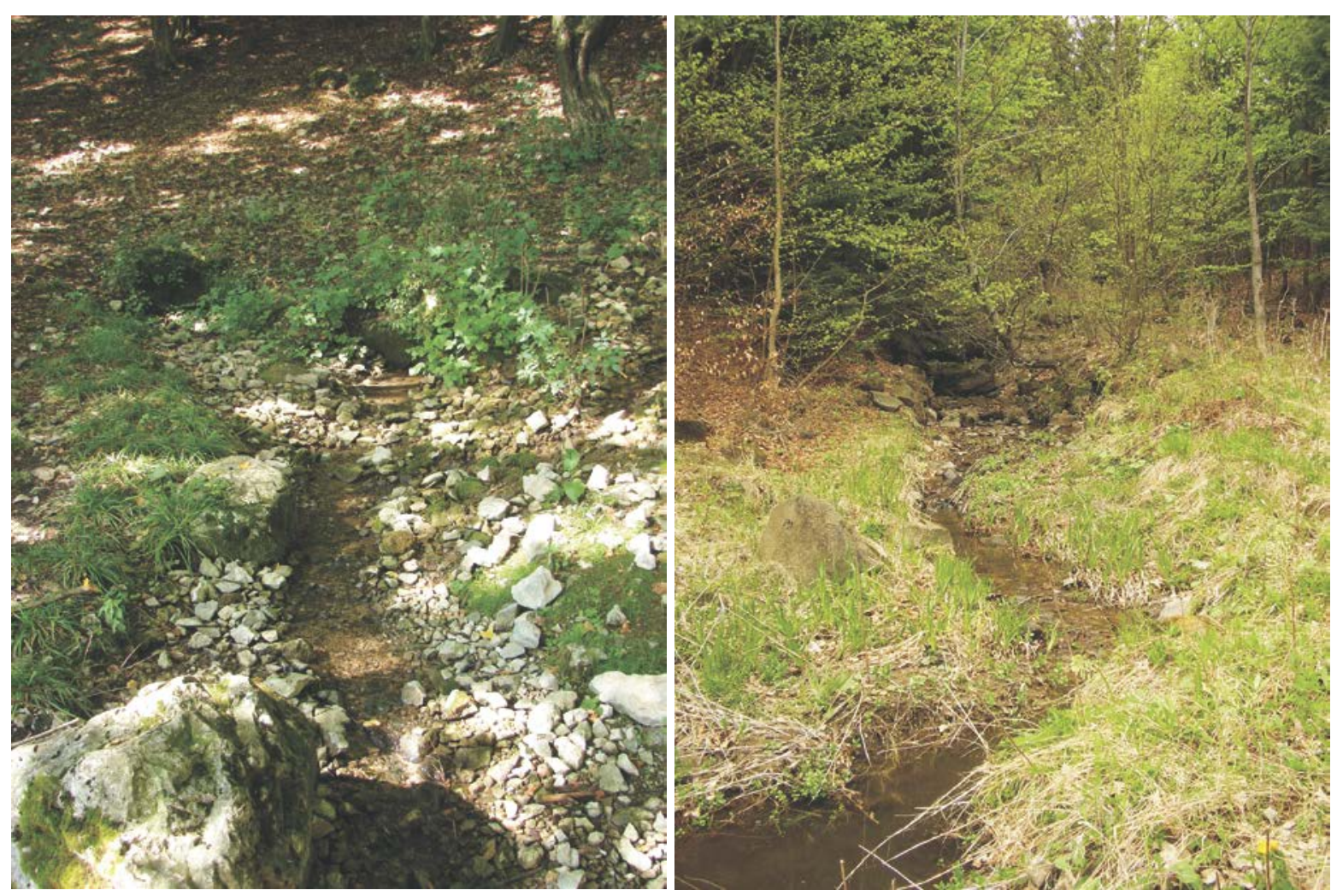

Figs 10-11: 10 - Trstenec, well and small brook in oak forest (photo by Jozef Oboňa), 11 - Železná Breznica, brook in beech forest (photo by Vladimír Kubovčík). 


\section{Discussion}

This prodromus summarizes all credible and available data about occurrence of nonbiting moth flies (Diptera: Psychodidae) from Slovakia. Data from more then 50 papers (larvae and adults occurrence) were excerpted and approximately 400 localities were accounted together with random and extensive new unpublished sampling. First records of fourteen new species for the fauna of Slovakia increased the species-richness of moth flies to 117 species in this country. As already indicated, the investigation of Psychodidae in Slovakia is still far from finished. This argument is supported by several papers, which examined Psychodidae fauna in the immediate vicinity of Slovakia state borders. Ježek \& Omelková 2007 published Telmatoscopus britteni Tonnoir, 1940 from Vrbovce env. (grid mapping code of DFS - 7171) only approximately three hundred meters far from borders; Ježek \& Omelková 2007 and 2012 published from Biele Karpaty Mts. (the same code of DFS) Jungiella (Psychocha) procera Krek, 1971, Mormia revisenda (Eaton, 1893) and Panimerus denticulatus Krek, 1971. Non-random (scheduled) and intensive sampling in Slovakia can detect many more interesting faunistic results in future. Unfortunately, some records of larvae (e.g. Berdeniella helvetica (Sara, 1957), B. illiesi Wagner, 1973 and Pericoma (Pericoma) diversa Tonnoir, 1919), have not been collected in the Slovakia as adults so far, but 3 species recorded on the basis of larvae Berdeniella manicata, Pericoma (Pericoma) pseudoexquisitaa and Pneumia stammeri are first time, in this paper, confirmed as adults from Slovakia.

Only 2 species (Sycorax slovaca and Trichomyia urbica) of family Psychodidae have been included into Red Data Book (Jedlička \& Stloukalová 2001) and opinions concerning the protection are being stepwise developed and they are varying with further findings and with gaining knowledge of biology of particular species. From 2001 Red Book has not been updated. With an increasing number of psychodid species from Slovakia is needed revaluate conservation status of concurrently known species. Our proposal is inspired by Ježek 2005 and Ježek et al. 2013. The Red list of threatened Psychodidae species in the Czech Republic (Ježek 2005) supplemented by recent studies (Omelková \& Ježek 2012b, Ježek et al. 2013) comprises 1 species as regionally extinct, 26 species as critically endangered (CR), 16 species as endangered (EN), 19 species as vulnerable (VU) and 12 species as nationally scarce (NS). Altogether 74 (from 173 species known from Omelková \& Ježek 2012b) species representing $42,8 \%$ of all species of this family known from the Czech Republic.

Under the criteria of IUCN (The International Union for the Conservation of Nature and Natural Resources) an only minimum number of psychodid species, 51 from 117 species, (representing 43,6 \% of all species of this family known from Slovakia) were assessment of the current conservation status and included into the list based on knowledge of the authors 24 species as critically endangered (CR) and 9 species as endangered (EN). Very rare species, described and frequently known only based on an unique specimen or on several specimens (18 species), are presented in the list below as vulnerable (VU - 3 species) or nationally scarce (NS - 15 species), in spite of the fact that many of them will be included in the future (based on stepwise growing data on their bionomics and distribution) into categories $\mathrm{CR}$ and EN.

Differences between this proposing and Red List Ježek (2005) in categorie CR are caused by a recent findings of species $P$. (P.) ljubiniensis and T. tatrica in Czech Republic (Ježek \& Omelková 2007) or absence of species T. negrobovi in Czech checklist. Species incorporated in category NS represent only national status, thus comparison with Czech Rebublic is irrelevant.

As dangerous appears to be species Clogmia albipunctata. This invasive, often synanthropic species may pose a risk for native synanthropic species and also is epidemiological significant as possible causal agent of various myiasis (e.g. Oboňa \& Ježek 2012a, Šuláková et al. 2014). As possible vectors of larval stages of Rhabditis nematodes and 
Gamasidae mites are species Psycha grisescens, Psychoda phalaenoides, Psychomora trinodulosa and Ypsydocha setigera, a protozoan parasite, a species of Glaucoma, was confirmed in Logima albipennis, Psychodocha gemina and P. cinerea (e.g. Satchell 1947, Ježek \& Omelková 2012a).

\section{A preliminary proposal of Slovak non-biting moth flies (Diptera: Psychodidae) to the new list of threatened species}

Critically Endangered (CR): Berdeniella helvetica (Sara, 1957), Clytocerus (Boreoclytocerus) rivosus (Tonnoir, 1919), Clytocerus (Boreoclytocerus) tetracorniculatus Wagner, 1977, Jungiella (Psychocha) laminata (Szabó, 1960), Lepiseodina tristis (Meigen, 1830), Oomormia andrenipes (Strobl, 1910), Parajungiella ellisi (Withers, 1987), Parajungiella pseudolongicornis (Wagner, 1975), Parajungiella serbica (Krek, 1985), Pericoma (Pericoma) ljubiniensis Krek, 1969, Peripsychoda fusca (Macquart, 1826), Philosepedon (Trichosepedon) balkanicum Krek, 1971, Promormia silesiensis Ježek, 1983, Psycmera integella (Jung, 1956), Saraiella parva (Vaillant, 1963), Saraiella rotunda (Krek, 1970), Sciria advena (Eaton, 1893), Sycorax slovaca Halgoš, 1975, Sycorax tonnoiri Jung, 1954, Szaboiella hibernica (Tonnoir, 1940), Taramormia tatrica Ježek, 1984, Threticus negrobovi Vaillant, 1972, Tonnoiriella nigricauda (Tonnoir, 1919), Trichomyia urbica Curtis, 1839

Endangered (EN): Pericoma (Pericoma) calcilega Feuerborn, 1923, Pericoma (Pericoma) exquisita Eaton, 1893, Pneumia crispi (Freeman, 1953), Pneumia cubitospinosa (Jung, 1954), Pneumia rivularis Berdén, 1954, Promormia eatoni (Tonnoir, 1940), Threticus incurvus Krek, 1972, Tonnoiriella sieberti Wagner, 1993, Ulomyia vaseki Ježek, 2002

Vulnerable (VU): Parajungiella prikryli Ježek, 1999, Telmatoscopus carpathicus Ježek, 1988, Threticus silvaticus Ježek, 1985

Nationally Scarce (NS): Berdeniella gardinii Salamanna \& Raggio, 1984, Berdeniella kocii Ježek, 2006, Chodopsycha buxtoni (Withers, 1988), Clytocerus (Boreoclytocerus) longicorniculatus Krek, 1987, Clytocerus (Boreoclytocerus) splendidus Ježek \& Hájek, 2007, Paramormia (Phyllotelmatoscopus) acuta (Krek, 1971), Pericoma (Pericoma) diversa Tonnoir, 1919, Pericoma (Pericoma) pallida Vaillant, 1978, Pericoma (Pericoma) pingarestica Vaillant, 1978, Pericoma (Pericoma) tatrica Szabó, 1960, Pericoma (Pericoma) tonnoiri Vaillant, 1978, Pericoma (Pericoma) vestita Vaillant et Withers, 1993, Pneumia compta (Eaton, 1893), Pneumia fonticola (Szabó, 1960), Pneumia kabelaki Omelková \& Ježek, 2012

Acknowledgements: We would like to thank Eva Bulánková (Comenius University, Bratislava, Slovakia) and Vladimír Straka (Slovak National Museum Martin, Slovakia) for provides us a faunistic literature. Thanks are also due to Jiř́ Burda, Pavel Chvojka and Petr Kment (all National Museum, Praha, Czech Republic), Pavel Lauterer (Zemské Moravské Muzeum, Brno, Czech Republic), Jan Ševčík (University of Ostrava, Ostrava, Czech Republic), Martin Baláž (Trebatice, Slovakia), Ludmila Balážiová (Pieštany, Slovakia), Mário Dobránsky (Vel'ké Kapušany, Slovakia), Peter Filipovič (Rastislavice, Slovakia), Vladimír Košel (Comenius University, Bratislava, Slovakia), Jozef Lukáš (Comenius University, Bratislava, Slovakia), Karol Ox (Košice, Slovakia), Vladimír Straka (see above) and Marek Svitok (Technical University in Zvolen, Zvolen, Slovakia) for their activities in the field or providing us with very interesting material in their charge. We are grateful to Michal Tkoč (National Museum, Praha, Czech Republic) - technical help with scanning of figures, Vladimír Kubovčík, Marek Svitok (both Technical University in Zvolen, Zvolen, Slovakia) and Miloš Villaris (Pieštany, Slovakia) for their photographs of important localities and all anonymous reviewers for their valuable and instructive comments on the manuscript. This work was supported by the Slovak Research and Development Agency under the contract No. APVV-0059-11, by grant OPV ITMS: 26110230119 and partly supported by the Ministry of Culture of the Czech Republic (DKRVO 2014/12, National Museum, 0002327201). 


\section{References}

Bitušík P., Bulánková E., Illéšová D. \& Halgoš J. (2000): Ekologické hodnotenie potokov v BR Pol’ana na základe preimaginálných štádií vodných dvojkrídlovcov (Diptera). In Midriak R. \& Sláviková D. (eds): Biosférické rezervácie na Slovensku - III. Zborník referátov z 3. národnej konferencie o biosférických rezerváciách SR, Zvolen, pp. 30-39.

Bulánková E. (1991): Faunistical notes (Diptera, Tipulidae, Psychodidae). - Biológia 46 (10): 970.

- (1999): Výskyt vybraných skupín vodných dvojkrídlovcov v BR Pol’ana. [Aquatic Diptera (excl. Chironomidae, Simuliidae) of Pol'ana Biospheric Reserve (Slovakia)]. - Folia faun. Slov. 4: 151-156.

- (2002): Dipteran communities (excl. Chironomidae, Simuliidae) of the Gidra river basin. - Acta Zool. Univ. Comen. 45: 77-86.

- (2003): Súpis druhov vodných bezstavovcov (makroevertebrát) Slovenska, Kapitola 2 - iné Diptera. In Šporka F. (ed.): Vodné bezstavovce (makroevertebráta) Slovenska. Súpis druhov a autekologické charakteristiky. Slovenský hydrometeorologický ústav, Bratislava, pp. 70-74.

- (2007): Microhabitat distribution of Diptera larvae (excl. Chironomidae, Simuliidae) in a small Carpathian stream. - Acta Zool. Univ. Comen. 47(2): 127-135.

Bulánková E. \& Degma P. (1996): Flies (Diptera) (excl. Chironomidae, Simuliidae), pp. 70-81. In: Krno I. (ed.) Limnology of the Turiec river basin (West Carpathians, Slovakia), Biologia, Bratislava 51, Suppl. 2.

Bulánková E. \& Halgoš J. (1995): Príspevok k poznaniu druhov vybraných čel’adí dvojkrídlovcov (Diptera) pohoria Vtáčnik. - Rosalia 10: 145-150.

Bulánková E., Halgoš J. \& Illéšová D. (2001): Changes of the Diptera taxocoenoses in the longitudinal profile on the Higra stream basin (The Little Carpathians). - Acta Univ. Carol. Biol. 45: 19-24.

Bulánková E., Halgoš J., Krno I., Bitušík P., IIléšová D., Lukáš J., Derka T. \& Šporka F. (2002): The influence of different thermal regime on the structure of coenoses of stenothermal hydrobionts in mountain streams. - Acta Zool. Univ. Comen. 44: 95-101.

Bulánková E. \& Némethová D. (2007): Linkages among land-use, water quality, physical habitat conditions and selected macroinevertebrate assemblages of the Hron River (Slovakia). - Acta Univ. Carol. Environ. 21: 35-46.

Bulánková E. \& Zat’ovičová Z. (2006): Preimaginal stages of Diptera (excluding Chironomidae and Simuliidae) of mountain lakes, their inlets and outlets in the Tatra Mountains (Slovakia). - Biologia 61/Suppl. 18: 185-190.

Enderlein G. (1937): Klassifikation der Psychodiden (Dipt.). - Dtsch. Ent. Z., Berlin, 1936: 81-112.

Halgoš J. (1973): Príspevok k poznaniu rozšírenia druhov čel'ade Psychodidae (Diptera, Nematocera) na územi západného Slovenska. [Contribution to the knowledge of the distribution of species of family Psychodidae (Diptera, Nematocera) in west Slovakia]. - Acta F.R.N. Univ. Comen - Zool. 19: 71-77.

- (1974): Dva druhy rodu Sycorax (Diptera, Psychodidae) nové pre faunu Československa. [Two Sycorax species (Diptera, Psychodidae) new to Czechoslovakia]. - Biológia 29(5): 437-439.

- (1975): Description of Sycorax slovacus sp. n. (Diptera, Psychodidae). - Ann. Zool. Bot. 106: 1-4.

- (1984): Psychodidae. pp. 48-51. In. Čepelák J. (ed.) Diptera Slovenska I (Nematocera, Brachycera Orthorrhapha). Veda, Bratislava, 288 pp.

Ibáñez-Bernal S. (2008): New records and descriptions of Mexican moth flies (Diptera: Psychodidae, Psychodinae). - Trans. Amer. Ent. Soc. 134: 87-131.

Jedlička L. \& Stloukalová V. (2001): Červený (ekosozoologický) zoznam dvojkrídlovcov (Diptera) Slovenska. In: Baláž D., Marhold K. \& Urban P. (eds) Červený zoznam rastlín a živočíchov Slovenska. - Ochrana prírody, Suppl. 20: 139-142.

Ježek J. (1977): Reinstatement of the genus Tinearia Schellenberg (Diptera, Psychodidae). - Acta Ent. Bohemoslov. 74: 232-241.

- (1983): Contribution to the taxonomy of the genus Logima Eat. (Diptera, Psychodidae). - Acta Ent. Mus. Nat. Pragae 41: 213-234.

- (1984a): A taxonomic study of the genera Psycmera Jez. and Parajungiella Vaill. (Diptera, Psychodidae) of Czechoslovakia. - Acta Mus. Nat. Pragae, B 40: 1-19.

- (1984b): Taxonomic notes on Mormiini (Diptera, Psychodidae) from the High Tatra National Park. - Acta Ent. Bohemoslov. 81: 223-231.

- (1987): Jungiella hygrophila sp. n. (Diptera, Psychodidae, Paramormiini) with redescriptions of Czechoslovak species of Jungiella s. str. - Acta Ent. Mus. Nat. Pragae, 42: 207-223.

- (1989): Contribution to the taxonomy of the genus Telmatoscopus Eat. (Diptera, Psychodidae). - Acta Mus. Nat. Pragae 44 B (1988): 75-104.

- (1990a): Contribution to the taxonomy of some genera of Paramormiine moth flies (Diptera, Psychodidae) with description of a new genus Karakovounimerus. - Acta Ent. Mus. Nat. Pragae 43: 129-157.

- (1990b): Redescriptions of nine common palaearctic and holarctic species of Psychodini End. (Diptera: Psychodidae). - Acta Ent. Mus. Nat. Pragae 43: 33-83. 
- (1993): Notes on faunistic research of moth flies (Diptera, Psychodidae) of Tatra Mts. - Dipt. Bohemoslov. 5: 35-40.

- (1994): Katalóg taxonů subtribu Mormiina End. Holarktické a Ethiopské oblasti (Diptera, Psychodidae, Psychodinae, Mormiini) [Catalogue of Holarctic and Afrotropical Mormiina End. (Diptera, Psychodidae, Psychodinae, Mormiini)]. - Čas. Nár. Muz., Ř. Př́rodov. 162: 63-66.

- (1995): Occasional paper on some interesting Palaearctic moth flies (Diptera, Psychodidae). In Bitušík P. (ed.). - Dipt. Bohemoslov. 7: 85-96.

- (1997): Redescription and figures of males of two little known Tonnoir's species of moth flies (Diptera, Psychodidae). - Dipt. Bohemoslov. 8: 63-71.

- (1999): Moth flies (Diptera, Psychodidae) inhabiting wet extreme biotopes of the Sokolov open coal mines and dumps, with descriptions of three new species from the western Bohemia. - Acta Mus. Nat. Pragae, 54 B (1998): 45-60.

- (2002): The first account of Slovenian moth flies (Psychodidae, Diptera). - Čas. Nár. Muz., Ř. Př́rodov. 171: 89-116.

- (2003): New faunistic data and check list of non Phlebotomine moth flies (Diptera, Psychodidae) from the Czech and Slovak republics. - Čas. Nár. Muz., Ǩ. Př́rodov. 172: 121-132.

- (2005) Čeled': Psychodidae (koutulovití), pp. 259-261. In: Farkač J., Král D. \& Škorpík M. (eds), Červený seznam ohrožených druhů České republiky. Bezobratlí. Red list of threatened species in the Czech Republic. Invertebrates. Agentura ochrany prírody a krajiny ČR, Praha, 760 pp.

- (2006a): New faunistic records of moth flies (Insecta, Diptera, Psychodidae) from Slovakia. - Ent. Carpath. 18: 34-36.

- (2006b): Faunistic records, Psychodidae. In: Kinkorová J. (ed.). - Dipt. Bohemoslov. 13: 139-141.

- (2007): New records of moth flies (Diptera, Psychodidae) from Poland with description of Apsycha gen. nov. Acta Zool. Univ. Comen. 47: 145-160.

- (2009a): Psychodidae, pp. 89-92. In Roháček J. \& Ševčík J. (eds): Diptera of the Pol’ana Protected Landscape Area - Biosphere Reserve (Central Slovakia). SNC SR, Administration of the PLA - BR Pol'ana, Zvolen, 340 pp.

- (2009b): Further new faunistic records of moth flies (Insecta, Diptera, Psychodidae) from the Czech Republic and Slovakia. - Folia Faun. Slov. 14(15): 101-105.

- (2009c): Checklist of Diptera of the Czech Republic and Slovakia, electronic version 2: Psychodidae Newman, 1834. Available from http://zoology.fns.uniba.sk/diptera2009/Psychodidae.htm (accessed 2.4.2014).

Ježek J. \& Goutner V. (1995): Psychodidae (Diptera) of Greece. - Acta Mus. Nat. Pragae 50B (1994): $107-$ 124.

Ježek J. \& Hájek J. (2007): Psychodidae (Diptera) of the Orlické hory Protected Landscape Area and neighbouring areas with descriptions of two new species from the Czech Republic. - Acta Ent. Mus. Nat. Pragae 47: 237-285.

Ježek J. \& Halgoš J. (1987): Psychodidae, pp. 29 - 32. In Ježek J. (ed.) Enumeratio insectorum Bohemoslovakiae. Check list of Czechoslovak insects 2 (Diptera). - Acta Faun. Ent. Mus. Nat. Pragae 18: 1-341.

Ježek J., Kubík Š. \& Barták M. (2013): Psychodidae (Diptera) of Vráž nr. Písek (Czech Republic), pp. 189199. In: Kubík Š. \& Barták M. (eds.): Workshop on biodiversity, Jevany. Česká zemědělská univerzita v Praze. 436 pp.

Ježek J., Lukáš J., Kvifte G.M. \& Oboňa J. (2012): New faunistic records of non-biting moth flies (Diptera: Psychodidae) from the Czech Republic and Slovakia. - Klapalekiana 48: 121-126.

Ježek J. \& Omelková M. (2007): Faunistic records from Czech Republic and Slovakia: Diptera, Psychodidae. - Acta Zool. Univ. Comen. 47: 250-253.

Ježek J. \& Omelková M. (2012): Moth flies (Diptera: Psychodidae) of the Bílé Karpaty Protected Landscape Area and Biosphere Reserve (Czech Republic). In Malenovský I., Kment P. \& Konvička O. (eds.): Species inventories of selected insect groups in the Bílé Karpaty Protected Landscape Area and Biosphere Reserve (Czech Republic). - Acta Mus. Morav., Sci. Biol. 96(2): 763-802.

Ježek J. \& Schacht W. (2006): Moth Flies from Bavaria (Diptera, Psychodidae). - Entomofauna. Zeitschr. Ent. 38: 477-484.

Ježek J. \& van Harten A. (2009): Order Diptera, Family Psychodidae. Subfamily Psychodinae (non-biting moth flies), pp. 686-711. In: van Harten A. (ed.): Arthropod Fauna of the United Arab Emirates, Vol. 2. Dar Al Ummah Printing, Publishing, Distribution and Advertising, Abu Dhabi, 786 pp.

Košel V. \& Horváth M. (1996): Temporal and special dynamics of Nematocera (Insecta, Diptera) in a cave of the Western Carpathians (Slovakia). - Acta Zool. Univ. Comen. 40: 75-114.

Krek, S. (1999) Psychodidae (Diptera, Insecta) Balkanskog Poluotoka. Federacija Bosne i Hercegovine, Ministarstvo Obrazovanja, Nauke, Kulture i Sporta, Studentska Štamparija Univerziteta Sarajevo, Sarajevo, 417 pp. 
Krno I. (1992): Makrozoobentos pramenisk v CHKO Velka Fatra. - Ochrana prírody 1: 107-116.

Krno I., Bulánková E. \& Halgoš J. (1994): Macrozoobenthos of the Morava river basin and tributaries of the Morava. - Ecology, Suppl. 1994 (1): 63-76.

Krno I., Tomajka J., Tiriaková E., Bulánková E., Halgoš J. \& Košel V. (1995): Vplyv kyslých zrážok na faunu pramenísk pohoria Vtáčnik. - Rosalia 10: 21-34.

Kvifte G.M. \& Andersen T. (2012): Moth flies (Diptera, Psychodidae) from Finnmark, northern Norway. Norwegian J. Ent. 59: 108-119.

Kvifte G.M., Ivković M. \& Klarić A. (2013): New records of moth flies (Diptera: Psychodidae) from Croatia, with the description of Berdeniella keroveci sp.nov. - Zootaxa, 3737 (1): 57-67.

Lučivianská V. (1989): Lokality. [Localities]. pp. 8-126. In: Čepelák J. (ed.): Diptera Slovenska, III. [Slovak Diptera, III]. Veda, Bratislava, 192 pp.

Mayer K. (1938): Zur Kenntnis der Buchenhöhlenfauna. - Arch. Hydrobiol. 33: 388-400.

Mazúr E., Lukniš M., Balatka B., Loučková J. \& Sládek J. (1986): Geomorfologické členenie SSR a ČSSR. Mapa mierky 1:500 000, Slovenská kartografia, SUGK, Bratislava.

Mišíková Elexová E., Haviar M., Lešt’áková M., Ščerbáková S., Bitušík P., Bulánková E., Čejka T., Čiamporová-Zat’ovičová Z., Derka T., Hamerlík L., Illéšová D., Kodada J., Košel V., Krno I., Mláka M., Novikmec M. \& Šporka F. (2010): Zoznam zistených taxónov na monitorovaných lokalitách vodných útvarov povrchových vôd Slovenska. Čast' 1 - Bentické bezstavovce. - Acta Environ. Univ. Comen. 18 (1): 1-335.

Novikmec M., Svitok M., Bulánková E., Čiamporová-Zat’ovičová Z., Derka T., Halgoš J., Hamerlík L., Illéšová D., Illyová M., Krno I., Lukáš J., Némethová D., Pastuchová Z., Stašiov S., Šporka F., Štefková E., Tirjaková E., Tomajka J. \& Bitušík P. (2007): Limnology of streams in the Poloniny National Park (the East Carpathians, Slovakia). Technical University in Zvolen, 69 pp.

Oboňa J. \& Ježek J. (2012a): Range expansion of the invasive moth midge Clogmia albipunctata (Williston, 1893) in Slovakia (Diptera: Psychodidae). - Folia Faun. Slov. 17 (4): 387-391.

- (2012b): First records of dendrolimnetic moth flies (Diptera: Psychodidae) from Slovakia. - Klapalekiana 48: 279-287.

Omelková M. \& Ježek J. (2012a): Two new species of Pneumia Enderlein (Diptera, Psychodidae, Psychodinae) from the Palaearctic region. - Zootaxa 3180: 1-18.

- (2012b): A new species of the genus Trichomyia (Diptera: Psychodidae) and new faunistic data on nonphlebotomine moth flies from the Podyjí NP and its surroundings (Czech Republic). - Acta Mus. Morav., Sci. Biol. 55(2): 505-533.

Rozkošný R. (1971): To the knowledge of Psychodidae (Diptera) in Czechoslovakia. - Scripta Fac. Sci. Nat. UJEP Brun., Biol. 2, 1: 133-144.

Salamanna G. \& Sarà M. (1980): Psicodidi delle Dolomiti (Diptera Nematocera). - Estratto dalle Mem. Soc. Ent. Ital. 58: 9-40.

Satchell G.H. (1947): The ecology of the British species of Psychoda (Diptera: Psychodidae). - Ann. Appl. Biol. 34: 611-621.

Ševčík J. (2004): Diptera associated with fungi in the Poloniny National Park (Bukovské vrchy Mts., East Slovakia). - Folia Fac. Sci. Nat. Univ. Masaryk. Brun., Biol. 109: 293-304.

- (2010): Czech and Slovak Diptera associated with fungi. Slezské zemské muzeum Opava, 112 pp.

Števove B. \& Bulánková E. (2010): Macrozoobenthos of the middle part of the Vydrica stream - comparison after 50 and 25 years. - Folia Faun. Slov. 15: 19-24.

Stloukal E. \& Grujbárová Z. (2013): Databáza lokalít pre mapovanie fauny na území Slovenska. $<$ http://www.dfs.sk $>$. (accessed 2.4.2014).

Straka V. (2010): Dvojkrídlovce (Diptera) Lukovského vrchu v Chránenej krajinnej oblasti Biele Karpaty [Diptera of the Lukovský vrch hill in the Protected Landscape Area Biele Karpaty Mts. (West Slovakia)]. Naturela Tutela 14: 45-54.

- (2009): Dvojkrídlovce (Diptera) vybraných lokalít v Považskom podolí a v Trnavskej pahorkatine [Flies (Diptera) of selected localities in Považské podolie basin and in Trnavská pahorkatina highlands (western Slovakia)]. - Naturela Tutela 13: 83-91.

Straka V. \& Majzlan O. (2006): Fauna dvojkrídlovcov (Diptera) prírodnej rezervácie Nad Šenkárkou v CHKO Malé Karpaty (Južné Slovensko) [The fauna Diptera of the Nature Reserve Nad Šenkárkou in the Protected Landscape Area Malé Karpaty (south Slovakia)]. - Naturela Tutela 10: 11-31.

- (2007): Dvojkrídlovce (Diptera) troch lokalít v chránenej krajinnej oblasti Strážovské vrchy [Flies (Diptera) of three localities in the Strážovské vrchy hills Protected Landscape Area]. - Naturela Tutela 11: 47-84.

- (2009): Dynamika abundancie dvojkridlovcov (Diptera) vo Vysokych Tatrach (Eklektorfauna). - Študie o Tatranskom Národnom Parku 9(42): 183-190.

- (2010a): Dvojkrídlovce (Diptera) PR Šúr. In: Majzlan O. \& Vidlička L. (eds), Príroda a rezervácie Šúr. pp. 285-324. 
- (2010b): Dynamika abundancie dvojkrídlovcov (Diptera) na území národnej prírodnej rezervácie Bábsky les pri Nitre (južné Slovensko) [Dynamics of the flies abundance (Diptera) in the National Nature Reserve Bábsky les near Nitra (South Slovakia)]. - Rosalia 21: 167-184.

- (2013): Dvojkrídlovce (Diptera) na lokalite Šišulákov mlyn pri obci Závod v Záhorskej nížine [Flies (Diptera) in the locality Šišulákov mlyn (mill) near Závod community in Záhorská nížina (lowlands) (west Slovakia)]. - Naturela Tutela 17: 89-104.

Szabó J. (1960): Neue, sowie aus dem Karpatenbecken bisher nicht nachgewiesene Psychodiden-Arten (Diptera, Nematocera). - Acta Zool. Acad. Sci. Hung. 6(3-4): 419-428.

- (1965a): Beiträge zur Verbreitung der Psychodiden (Diptera, Nematocera), in der Slowakei. - Acta ent. Mus. Nat. Pragae 36: 607-631.

- (1965b): Beiträge zur Kenntnis der Psychodiden-fauna (Diptera, Nematocera) im östlichen Teil der Tsechoslowakei. - Acta Biol. Debrec. 3: 69-92.

- (1972-73): Marpho-Ökologische Untersuchungen an Psychodiden-Larven (Diptera, Nematocera). I. Die Haut (Integumentum) und ihre Anhänge. - Acta Biol. Debrec. 10-11: 163-189.

Šuláková H., Gregor F., Ježek J. \& Tkoč M. (2014): Nová invaze do našich obcí a měst: koutule Clogmia albipunctata a problematika myiáz. - Živa 1: 29-32.

Thalhammer J. (1918): Fam. Psychodidae, p. 16. Ordo Diptera. In Fauna Regini Hungariae, Budapest, 76 pp.

Tonnoir A.L. (1940): A synopsis of the British Psychodidae (Dipt.), with descriptions of new species. - Trans. Soc. Brit. Ent.. 7: 21-64.

Vaillant F. (1971-1983): 9d. Psychodidae - Psychodinae (not finished). In: Lindner E. (ed.): Die Fliegen der palaearktischen Region. Stuttgart. Vols. 287(1971): 1-48; 291 (1972): 49-78; 292(1972): 79-108; 305(1974): 109-142; 310(1975): 143-182; 313(1977): 183-206; 317(1978): 207-238; 320(1979): 239-270; 326(1981): 271-310; 328(1983): 311-357.

Wagner R. (1977): Zur Kenntnis der Psychodidenfauna des Allgäus (Diptera: Nematocera). - Nachr. Bayer. Ent. 26: 23-28.

- (2013): Fauna Europaea: Psychodidae. In: de Jong H. (ed.): Fauna Europaea: Diptera Nematocera. Fauna Europaea version $2.4<$ http://www.faunaeur.org $>$. (accessed 2.4.2014).

Authors' addresses: Jozef Oboňa, Department of Ecology, Faculty of Humanities and Natural Sciences, University of Prešov, 17. novembra 1, SK - 08116 Prešov, Slovakia; e-mail: obonaj@centrum.sk

Jan Ježek, Department of Entomology, National Museum, Cirkusová 1740, CZ - 19300 Praha 9 - Horní Počernice, Czech Republic; e-mail: jan.jezek@quick.cz 